\title{
Enantioselective Rhodium-Catalyzed [2+2+2] Cycloaddition of Alkenyl Isocyanates and Terminal Alkynes: Application to the Total Synthesis of (+)-Lasubine II.
}

\author{
Robert T. Yu and Tomislav Rovis* \\ Department of Chemistry, Colorado State University \\ Fort Collins, Colorado 80523
}

\section{Supporting Information}

General Methods. All reactions were carried out under an atmosphere of argon in flame-dried glassware with magnetic stirring. Toluene was degassed with argon and passed through one column of neutral alumina and one column of Q5 reactant. Triethylamine (peptide synthesis grade) was purchased from Fisher Scientific and used without further purification. Column chromatography was performed on EM Science silica gel 60 (230-400 mesh). Thin layer chromatography was performed on EM Science $0.25 \mathrm{~mm}$ silica gel $60-\mathrm{F}$ plates. Visualization was accomplished with UV light and $\mathrm{KMnO}_{4}$ followed by heating.

Infrared spectra were obtained on a Nicolet Avatar 320 FT-IR spectrometer. ${ }^{1} \mathrm{H}$ NMR and spectra were recorded on a Varian 300 or $400 \mathrm{MHz}$ spectrometers at ambient temperature. Data are reported as follows: chemical shift in parts per million $(\delta, \mathrm{ppm})$ from deuterated chloroform $\left(\mathrm{CDCl}_{3}\right)$ taken as $7.26 \mathrm{ppm}(300 \mathrm{MHz})$ or $7.23 \mathrm{ppm}(400$ $\mathrm{MHz}$ ), multiplicity ( $\mathrm{s}=$ singlet, $\mathrm{d}=$ doublet, $\mathrm{t}=$ triplet, $\mathrm{q}=$ quartet, and $\mathrm{m}=$ multiplet), integration, and coupling constant $(\mathrm{Hz}) .{ }^{13} \mathrm{C}$ NMR and spectra were recorded on a Varian 300 or $400 \mathrm{MHz}$ spectrometers at ambient temperature. Chemical shifts are reported in ppm from $\mathrm{CDCl}_{3}$ taken as $77.0 \mathrm{ppm}$. Mass spectra were obtained on Fisons VG Autospec.

Alkynes 1a, 1c - 1g, 1j, 1l, 1n - 1s, 1u, and 1v were all purchased from Aldrich Chemicals Co. and used without further purification. Alkyne 1b, ${ }^{1}$ isocyanates $\mathbf{2}$ and $\mathbf{5}$ were prepared according to the literature methods. ${ }^{2}$ Alkyne 1t was prepared by a typical TBS-protection of the corresponding alcohol, which was purchased from Aldrich Chemicals Co. Alkynes $1 \mathbf{h}, \mathbf{1 k}, \mathbf{1 m}$ and ligands $\mathbf{L 4}$ and $\mathbf{L 5}$ are known compounds and can be synthesized by the procedure described within. Phosphorus trichloride and tris(4methoxyphenyl) phosphine were purchased from Aldrich Chemicals Co. $\left[\mathrm{Rh}(\text { ethylene })_{2} \mathrm{Cl}\right]_{2}, \mathrm{PdCl}_{2}\left(\mathrm{PPh}_{3}\right)_{2}$, ligands $\mathbf{L 1}-\mathbf{L 3}$, and trimethylsilylacetylene were purchased from Strem Chemical, Inc. and used without further purification. All racemates are obtained via the same cycloaddition using $r a c-\mathbf{L 3}$ as the ligand.

\footnotetext{
${ }^{1}$ Lawrence, N. J.; Ghani, F. A.; Hepworth, L. A.; Hadfield, J. A.; McGown, A. T.; Pritchard, R. G. Synthesis 1999, 1656.

${ }^{2}$ Yu, R. T.; Rovis, T. J. Am. Chem. Soc. 2006, 128, 2782.
} 
General procedure for synthesis of terminal aryl alkynes (1h as the example):
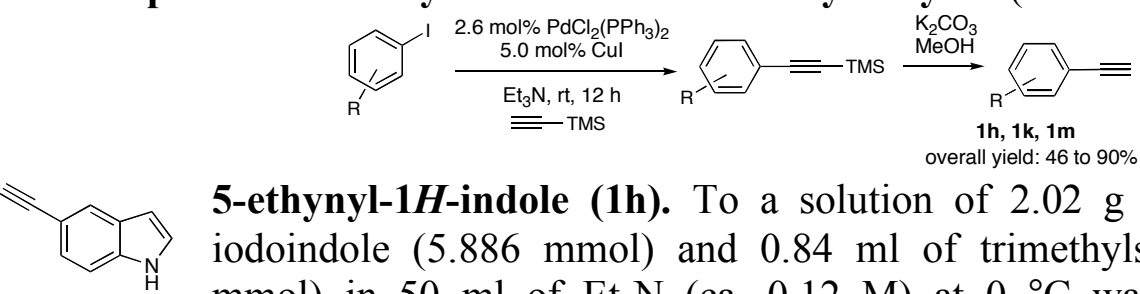

5-ethynyl-1H-indole (1h). To a solution of $2.02 \mathrm{~g}$ of Boc-protected 5iodoindole $(5.886 \mathrm{mmol})$ and $0.84 \mathrm{ml}$ of trimethylsilylacetylene $(5.886$ $\mathrm{mmol}$ ) in $50 \mathrm{ml}$ of $\mathrm{Et}_{3} \mathrm{~N}$ (ca. $0.12 \mathrm{M}$ ) at $0{ }^{\circ} \mathrm{C}$ was added $107 \mathrm{mg}$ of $\mathrm{PdCl}_{2}\left(\mathrm{PPh}_{3}\right)_{2}(0.153 \mathrm{mmol})$ and $56 \mathrm{mg}$ of $\mathrm{CuI}(0.294 \mathrm{mmol})$. The reaction mixture was stirred at ambient temperature for $12 \mathrm{~h}$. The reaction mixture was then filtered and concentrated in vacuo. The resulting slurry was dissolved in $20 \mathrm{ml}$ of $\mathrm{MeOH}$ (ca. $0.3 \mathrm{M}$ ), and treated with $1.62 \mathrm{~g}$ of $\mathrm{K}_{2} \mathrm{CO}_{3}(11.772 \mathrm{mmol})$. After stirring at ambient temperature for $2 \mathrm{~h}$, the mixture was diluted with $\mathrm{Et}_{2} \mathrm{O}$, partitioned with $\mathrm{H}_{2} \mathrm{O}$, dried $\left(\mathrm{MgSO}_{4}\right)$, and concentrated in vacuo. The crude material was purified by flash column chromatography (1:10 EtOAc/Hexane) to give the free indole $\mathbf{1 h}(400 \mathrm{mg}, 48 \%$ overall yield $): \mathrm{R}_{\mathrm{f}}=0.13$ (1:10 EtOAc/hex); ${ }^{1} \mathrm{H}$ NMR (400 MHz, $\left.\mathrm{CDCl}_{3}\right) \delta 8.20$ (br s, 1H), $7.82(\mathrm{~s}, 1 \mathrm{H}), 7.30-$ $7.34(\mathrm{~m}, 2 \mathrm{H}), 7.21(\mathrm{dd}, 1 \mathrm{H}, J=2.6,3.2 \mathrm{~Hz}), 6.52(\mathrm{dd}, 1 \mathrm{H}, J=2.1,3.0 \mathrm{~Hz}), 2.98(\mathrm{~s}, 1 \mathrm{H})$; ${ }^{13} \mathrm{C}$ NMR $\left(100 \mathrm{MHz}, \mathrm{CDCl}_{3}\right) \delta 135.8,127.8,126.2,125.5,125.3,113.4,111.3,103.1$, 85.5, 74.8; IR ( $\left.\mathrm{NaCl}, \mathrm{CDCl}_{3}\right)$ 3421, 3283, 1614, 1465, 1413, 1337, $1306 \mathrm{~cm}^{-1}$; HRMS $\left[\mathrm{C}_{10} \mathrm{H}_{7} \mathrm{~N}\right]^{+}$calcd 141.0578. Found $141.0583(\mathrm{FAB}+)$.

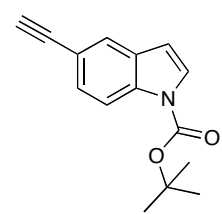

tert-butyl 5-ethynyl-1H-indole-1-carboxylate (1i). To a solution of 270 $\mathrm{mg}$ of $\mathbf{1 h}(1.91 \mathrm{mmol})$ and $7.0 \mathrm{mg}$ of DMAP $(0.057 \mathrm{mmol})$ in $2 \mathrm{ml}$ of dry acetonitrile (ca. $0.96 \mathrm{M})$ was added $0.48 \mathrm{ml}$ of $(\mathrm{Boc})_{2} \mathrm{O}(2.10 \mathrm{mmol})$ at ambient temperature. The reaction mixture was stirred at ambient temperature for $20 \mathrm{~h}$. The reaction mixture was concentrated in vacuo, partitioned with EtOAc/ $\mathrm{H}_{2} \mathrm{O}$, dried $\left(\mathrm{MgSO}_{4}\right)$, and concentrated in vacuo. The crude material was purified by flash column chromatography (1:10 EtOAc/Hexane) to give 1i as a yellow oil (409 mg, 89\% yield): $\mathrm{R}_{\mathrm{f}}=0.64$ (1:10 EtOAc/hex); ${ }^{1} \mathrm{H}$ NMR $(400 \mathrm{MHz}$, $\left.\mathrm{CDCl}_{3}\right) \delta 8.06(\mathrm{~d}, 1 \mathrm{H}, J=8.3 \mathrm{~Hz}), 7.69(\mathrm{~s}, 1 \mathrm{H}), 7.58($ br s, $1 \mathrm{H}), 7.41(\mathrm{~d}, 1 \mathrm{H}, J=8.7 \mathrm{~Hz})$, $6.51(\mathrm{~m}, 1 \mathrm{H}), 3.03(\mathrm{~s}, 1 \mathrm{H}), 1.66(\mathrm{~s}, 9 \mathrm{H}) ;{ }^{13} \mathrm{C} \mathrm{NMR}\left(100 \mathrm{MHz}, \mathrm{CDCl}_{3}\right) \delta 149.7,135.2$, 130.6, 128.3, 127.1, 125.3, 116.3, 115.3, 107.2, 84.5, 84.3, 76.0, 28.4; IR ( $\left.\mathrm{NaCl}, \mathrm{CDCl}_{3}\right)$ $3294,2979,1736,1462,1369,1339,1252,1159,1024 \mathrm{~cm}^{-1}$; HRMS $\left[\mathrm{C}_{15} \mathrm{H}_{16} \mathrm{NO}_{2}\right]^{+}$calcd 242.1181. Found 242.1183 (FAB+).

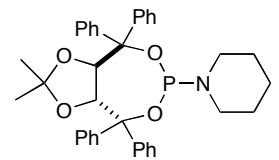

1-((3aR,8a $R)-2,2-d i m e t h y l-4,4,8,8$-tetraphenyltetrahydro$[1,3]$ dioxolo[4,5-e $][1,3,2]$ dioxaphosphepin-6-yl)piperidine (L4). To a flame-dried round bottom flask charged with a magnetic stir bar was added $4 \AA$ molecular sieves, followed by $500 \mathrm{mg}$ of (-)-TADDOL ${ }^{3}$ $(1.072 \mathrm{mmol})$ and $4.6 \mathrm{ml}$ of THF. To the reaction mixture was added $0.51 \mathrm{ml} \mathrm{of} \mathrm{Et}_{3} \mathrm{~N}$ (3.644 $\mathrm{mmol})$ and $0.112 \mathrm{ml}$ of phosphorus trichloride $(1.286 \mathrm{mmol})$ dropwise at $0{ }^{\circ} \mathrm{C}$. The mixture was allowed to warm to ambient temperature and stirred for 40 minutes. A solution of piperidine $(10.72 \mathrm{mmol})$ in $5.4 \mathrm{ml}$ of THF was added slowly at $0{ }^{\circ} \mathrm{C}$. The reaction was allowed to stir overnight at ambient temperature before it was diluted with

${ }^{3}$ Seebach, D.; Beck, A. K.; Keckel, A. Angew. Chem. Int. Ed. 2001, 40, 92. 
diethyl ether and filtered. The filtrate was concentrated in vacuo and the resulting crude material was purified by flash column chromatography (4:96 EtOAc/Hexane) to afford the desired phosphoramidite (400 mg, 65\% yield): $\mathrm{R}_{\mathrm{f}}=0.58(1: 10 \mathrm{EtOAc} / \mathrm{hex}) ;{ }^{1} \mathrm{H}$ NMR $\left(300 \mathrm{MHz}, \mathrm{CDCl}_{3}\right) \delta 7.78(\mathrm{~d}, 2 \mathrm{H}, J=7.3 \mathrm{~Hz}), 7.63(\mathrm{~d}, 2 \mathrm{H}, J=7.0 \mathrm{~Hz}), 7.48(\mathrm{~d}, 2 \mathrm{H}, J=$ $7.3 \mathrm{~Hz}), 7.42(\mathrm{~d}, 2 \mathrm{H}, J=7.3 \mathrm{~Hz}), 7.14-7.36(\mathrm{~m}, 12 \mathrm{H}), 5.16(\mathrm{~m}, 1 \mathrm{H}), 4.76(\mathrm{~d}, 1 \mathrm{H}, J=8.4$ $\mathrm{Hz}), 3.02-3.38(\mathrm{~m}, 4 \mathrm{H}), 1.50-1.68(\mathrm{~m}, 6 \mathrm{H}), 1.33(\mathrm{~s}, 3 \mathrm{H}), 0.29(\mathrm{~s}, 3 \mathrm{H})$.

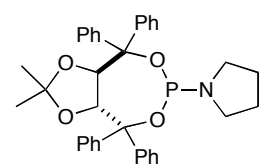

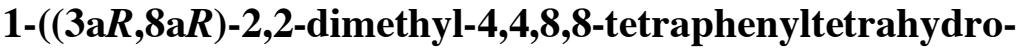
$[1,3]$ dioxolo[4,5-e $][1,3,2]$ dioxaphosphepin-6-yl)pyrrolidine (L5). To a flame-dried round bottom flask charged with a magnetic stir bar was added $4 \AA$ molecular sieves, followed by $1.20 \mathrm{~g}$ of (-)-TADDOL $(2.572$ mmol) and $11 \mathrm{ml}$ of THF. To the reaction mixture was added $1.23 \mathrm{ml}$ of $\mathrm{Et}_{3} \mathrm{~N}(8.745$ $\mathrm{mmol}$ ) and $0.27 \mathrm{ml}$ of phosphorus trichloride $(3.086 \mathrm{mmol})$ dropwise at $0{ }^{\circ} \mathrm{C}$. The mixture was allowed to warm to ambient temperature and stirred for 40 minutes. A solution of piperidine $(25.72 \mathrm{mmol})$ in $13 \mathrm{ml}$ of THF was added slowly at $0{ }^{\circ} \mathrm{C}$. The reaction was allowed to stir overnight at ambient temperature before it was diluted with diethyl ether and filtered. The filtrate was concentrated in vacuo and the resulting crude material was purified by flash column chromatography (4:96 EtOAc/Hexane) to afford the desired phosphoramidite (610 mg, $42 \%$ yield): $\mathrm{R}_{\mathrm{f}}=0.50(1: 10 \mathrm{EtOAc} / \mathrm{hex}) ;{ }^{1} \mathrm{H}$ NMR $\left(300 \mathrm{MHz}, \mathrm{CDCl}_{3}\right) \delta 7.68(\mathrm{~d}, 2 \mathrm{H}, J=7.0 \mathrm{~Hz}), 7.54(\mathrm{~d}, 2 \mathrm{H}, J=7.3 \mathrm{~Hz}), 7.42(\mathrm{~d}, 2 \mathrm{H}, J=$ $7.3 \mathrm{~Hz}), 7.35(\mathrm{~d}, 2 \mathrm{H}, J=7.3 \mathrm{~Hz}), 7.12-7.28(\mathrm{~m}, 12 \mathrm{H}), 5.15(\mathrm{dd}, 1 \mathrm{H}, J=2.9,8.4 \mathrm{~Hz})$, $4.76(\mathrm{~d}, 1 \mathrm{H}, J=8.4 \mathrm{~Hz}), 3.10-3.38(\mathrm{~m}, 4 \mathrm{H}), 1.66-1.82(\mathrm{~m}, 4 \mathrm{H}), 1.19(\mathrm{~s}, 3 \mathrm{H}), 0.23(\mathrm{~s}$, $3 \mathrm{H})$.

General procedure for the Rh-catalyzed [2+2+2] cycloaddition of alkenyl isocyanates and terminal alkynes: A flame-dried round bottom flask was charged with $\left.[\mathrm{Rh} \text { (ethylene) })_{2} \mathrm{Cl}\right]_{2}(0.05 \mathrm{eq})$ and the phosphoramidite ligand $\mathbf{L}(0.1 \mathrm{eq})$, and was fitted with a flame-dried reflux condenser in an inert atmosphere $\left(\mathrm{N}_{2}\right)$ glove box. Upon removal from the glove box, $1.0 \mathrm{ml}$ toluene was added via syringe and the resulting yellow solution was stirred at ambient temperature under argon flow for 15 minutes. To this solution was added a solution of alkyne $\mathbf{1}(2.0 \mathrm{eq})$ and isocyanate $\mathbf{2}$ or $5(0.270 \mathrm{mmol})$ in $2 \mathrm{ml}$ of toluene via syringe or cannula. After an additional $1 \mathrm{ml}$ of toluene to wash down the remaining residue, the resulting solution was heated to $110{ }^{\circ} \mathrm{C}$ in an oil bath, and maintained at reflux for $c a .16 \mathrm{~h}$. The reaction mixture was cooled to ambient temperature, concentrated in vacuo, and purified by flash column chromatography (gradient elution typically $100 \%$ ethyl acetate). Evaporation of solvent afforded the analytically pure product.

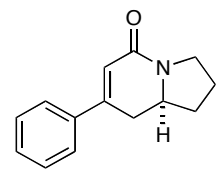

(S)-7-phenyl-2,3,8,8a-tetrahydroindolizin-5(1H)-one (3a). According to the general procedure with ligand L5, alkyne 1a, and isocyanate $\mathbf{2}$. Upon purification $4.6 \mathrm{mg}(8 \%)$ of the desired product was isolated: $\mathrm{R}_{\mathrm{f}}=0.21$ $($ EtOAc $) ;[\alpha]_{\mathrm{D}}{ }^{20}=+166.9\left(\mathrm{c}=0.16, \mathrm{CHCl}_{3}\right) ; \mathrm{HPLC}$ analysis - Chiracel AD-H column 90:10 hexane:iPrOH, $1.0 \mathrm{ml} / \mathrm{min}$, Major: 24.4 minutes, Minor: 19.6 minutes, $254 \mathrm{~nm}$ detection light, ee $=89 \%$; ${ }^{1} \mathrm{H} \mathrm{NMR}\left(400 \mathrm{MHz}, \mathrm{CDCl}_{3}\right) \delta 7.45-7.49$ $(\mathrm{m}, 2 \mathrm{H}), 7.33-7.40(\mathrm{~m}, 3 \mathrm{H}), 6.29(\mathrm{~d}, 1 \mathrm{H}, J=2.8 \mathrm{~Hz}), 3.81$ (dddd, $1 \mathrm{H}, J=5.1,5.1,10.2$, $14.9 \mathrm{~Hz}$ ), 3.67 (ddd, $1 \mathrm{H}, J=2.1,9.0,11.7 \mathrm{~Hz}), 3.51$ (ddd, $1 \mathrm{H}, J=7.7,9.4,11.5 \mathrm{~Hz}), 2.90$ 
(dd, $1 \mathrm{H}, J=4.7,16.6 \mathrm{~Hz}$ ), 2.51 (ddd, 1H, $J=2.8,14.1,16.6 \mathrm{~Hz}$ ), 2.27 (ddd, 1H, $J=5.3$, 5.3, $12.1 \mathrm{~Hz}), 2.06(\mathrm{~m}, 1 \mathrm{H}), 1.84$ (ddddd, $1 \mathrm{H}, J=6.6,9.6,9.6,12.2,12.2 \mathrm{~Hz}), 1.70(\mathrm{~m}$, $1 \mathrm{H}) ;{ }^{13} \mathrm{C} \mathrm{NMR}\left(100 \mathrm{MHz}, \mathrm{CDCl}_{3}\right) \delta 164.2,148.7,138.1,129.5,129.0,126.1,121.0,56.8$, 44.2, 33.7, 33.4, 23.3; IR ( $\left.\mathrm{NaCl}, \mathrm{CH}_{2} \mathrm{Cl}_{2}\right)$ 1644, 1593, 1450, 1352, $1327 \mathrm{~cm}^{-1}$; HRMS $\left[\mathrm{C}_{14} \mathrm{H}_{16} \mathrm{NO}\right]^{+}$calcd 214.1232. Found 214.1233 (FAB+).

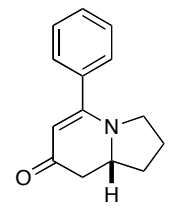

(R)-5-phenyl-2,3,8,8a-tetrahydroindolizin-7(1H)-one (4a). According to the general procedure with ligand L5, alkyne 1a, and isocyanate $\mathbf{2}$. From the same reaction as above, upon purification $44 \mathrm{mg}(79 \%)$ of the desired product was isolated: $\mathrm{R}_{\mathrm{f}}=0.15\left(\right.$ EtOAc); $[\alpha]_{\mathrm{D}}{ }^{20}=+640.0\left(\mathrm{c}=1.0, \mathrm{CHCl}_{3}\right)$; HPLC analysis - Chiracel OD-H column 85:15 hexane:iPrOH, 0.3 ml/min, Major: 49.9 minutes, Minor: 48.9 minutes, $330 \mathrm{~nm}$ detection light, ee $=94 \%$; ${ }^{1} \mathrm{H}$ NMR $(400$ $\left.\mathrm{MHz}, \mathrm{CDCl}_{3}\right) \delta 7.33-7.40(\mathrm{~m}, 5 \mathrm{H}), 5.05(\mathrm{~s}, 1 \mathrm{H}), 4.00(\mathrm{dddd}, 1 \mathrm{H}, J=7.2,7.2,7.2,14.3$ $\mathrm{Hz}$ ), 3.46 (ddd, $1 \mathrm{H}, J=4.5,7.7,11.5 \mathrm{~Hz}$ ), 3.23 (ddd, $1 \mathrm{H}, J=7.5,7.5,10.9 \mathrm{~Hz}$ ), 2.45 (dd, $1 \mathrm{H}, J=16.0,16.0 \mathrm{~Hz}), 2.40(\mathrm{dd}, 1 \mathrm{H}, J=6.7,16.2 \mathrm{~Hz}), 2.29(\mathrm{~m}, 1 \mathrm{H}), 1.98(\mathrm{~m}, 1 \mathrm{H}), 1.86$ $(\mathrm{m}, 1 \mathrm{H}), 1.75(\mathrm{~m}, 1 \mathrm{H}) ;{ }^{13} \mathrm{C}$ NMR $\left(100 \mathrm{MHz}, \mathrm{CDCl}_{3}\right) \delta 192.2,163.1,136.4,130.0,128.7$, 127.8, 100.2, 59.1, 49.5, 41.7, 32.2, 24.7; IR ( $\left.\mathrm{NaCl}, \mathrm{CH}_{2} \mathrm{Cl}_{2}\right)$ 1624, 1532, 1460, 1332 , 1260, $1235 \mathrm{~cm}^{-1}$; HRMS $\left[\mathrm{C}_{14} \mathrm{H}_{16} \mathrm{NO}\right]^{+}$calcd 214.1232. Found 214.1231 (FAB+).

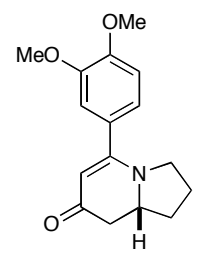

(R)-5-(3,4-dimethoxyphenyl)-2,3,8,8a-tetrahydroindolizin-7(1H)-one (4b). According to the general procedure with ligand L5, alkyne 1b, and isocyanate 2. Upon purification $53.2 \mathrm{mg}(72 \%)$ of the desired product was isolated: $\mathrm{R}_{\mathrm{f}}=0.05(\mathrm{EtOAc}) ;[\alpha]_{\mathrm{D}}{ }^{20}=+452.4\left(\mathrm{c}=1.0, \mathrm{CHCl}_{3}\right)$; HPLC analysis - Chiracel AD-H column 80:20 hexane:iPrOH, $1.0 \mathrm{ml} / \mathrm{min}$, Major: 24.2 minutes, Minor: 21.8 minutes, $330 \mathrm{~nm}$ detection light, ee $=94 \% ;{ }^{1} \mathrm{H}$ NMR (400 MHz, $\left.\mathrm{CDCl}_{3}\right) \delta 6.96(\mathrm{dd}, 1 \mathrm{H}, J=1.7,8.3 \mathrm{~Hz}), 6.88(\mathrm{br} \mathrm{s}, 1 \mathrm{H}), 6.85(\mathrm{~d}, 1 \mathrm{H}, J$ $=8.3 \mathrm{~Hz}), 5.19(\mathrm{~s}, 1 \mathrm{H}), 4.04(\mathrm{dddd}, 1 \mathrm{H}, J=7.0,7.0,7.0,13.4 \mathrm{~Hz}), 3.88(\mathrm{~s}, 3 \mathrm{H}), 3.86(\mathrm{~s}$, $3 \mathrm{H}), 3.54(\mathrm{ddd}, 1 \mathrm{H}, J=6.6,6.6,11.7 \mathrm{~Hz}), 3.27(\mathrm{ddd}, 1 \mathrm{H}, J=7.0,7.0,10.9 \mathrm{~Hz}), 2.46$ (dd, $1 \mathrm{H}, J=16.2,16.2 \mathrm{~Hz}), 2.38(\mathrm{dd}, 1 \mathrm{H}, J=5.8,16.2 \mathrm{~Hz}), 2.28(\mathrm{dddd}, 1 \mathrm{H}, J=6.4,6.4,6.4$, $12.8 \mathrm{~Hz}), 1.99(\mathrm{~m}, 1 \mathrm{H}), 1.88(\mathrm{~m}, 1 \mathrm{H}), 1.75(\mathrm{~m}, 1 \mathrm{H}) ;{ }^{13} \mathrm{C} \mathrm{NMR}\left(100 \mathrm{MHz}, \mathrm{CDCl}_{3}\right) \delta$ 192.1, 163.1, 150.6, 149.0, 128.9, 121.0, 111.1, 110.9, 100.0, 58.9, 56.2, 56.2, 50.0, 41.6, 31.9, 24.9; IR $\left(\mathrm{NaCl}, \mathrm{CDCl}_{3}\right)$ 1619, 1511, 1475, 1332, 1260, 1137, $922 \mathrm{~cm}^{-1}$; HRMS $\left[\mathrm{C}_{16} \mathrm{H}_{20} \mathrm{NO}_{3}\right]^{+}$calcd 274.1443. Found 274.1450 (FAB+).

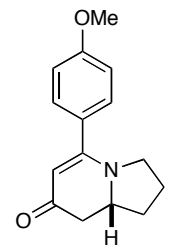

(R)-5-(4-methoxyphenyl)-2,3,8,8a-tetrahydroindolizin-7(1H)-one

(4c). According to the general procedure with ligand L5, alkyne 1c, and isocyanate 2. Upon purification $45.8 \mathrm{mg}(70 \%)$ of the desired product was isolated: $\mathrm{R}_{\mathrm{f}}=$ $0.05\left(\right.$ EtOAc); $[\alpha]_{\mathrm{D}}{ }^{20}=+462.4\left(\mathrm{c}=1.0, \mathrm{CHCl}_{3}\right)$; HPLC analysis - Chiracel OD-H column 80:20 hexane:iPrOH, $1.0 \mathrm{ml} / \mathrm{min}$, Major: 10.6 minutes, Minor: 12.1 minutes, $210 \mathrm{~nm}$ detection light, $e e=90 \% ;{ }^{1} \mathrm{H} \mathrm{NMR}\left(400 \mathrm{MHz}, \mathrm{CDCl}_{3}\right)$ $\delta 7.31(\mathrm{dm}, 2 \mathrm{H}, J=8.7 \mathrm{~Hz}), 6.88(\mathrm{dm}, 2 \mathrm{H}, J=8.9 \mathrm{~Hz}), 5.06(\mathrm{~s}, 1 \mathrm{H}), 4.02$ (ddd, $1 \mathrm{H}, J=$ 6.7, 6.7, 6.7, $13.5 \mathrm{~Hz}$ ), 3.80 (s, 3H), 3.53 (ddd, 1H, $J=5.5,7.3,11.8 \mathrm{~Hz}$ ), 3.25 (ddd, 1H, $J=7.0,7.0,10.7 \mathrm{~Hz}), 2.44(\mathrm{dd}, 1 \mathrm{H}, J=16.2,16.2 \mathrm{~Hz}), 2.37(\mathrm{dd}, 1 \mathrm{H}, J=5.6,16.2 \mathrm{~Hz})$, $2.27(\mathrm{~m}, 1 \mathrm{H}), 1.97(\mathrm{~m}, 1 \mathrm{H}), 1.86(\mathrm{~m}, 1 \mathrm{H}), 1.74(\mathrm{~m}, 1 \mathrm{H}) ;{ }^{13} \mathrm{C} \mathrm{NMR}\left(100 \mathrm{MHz}, \mathrm{CDCl}_{3}\right) \delta$ $192.1,163.0,161.1,129.5,128.8,114.0,100.0,58.9,55.6,49.8,41.7,31.9$, 24.8; IR 
$\left(\mathrm{NaCl}, \mathrm{CDCl}_{3}\right) 1624,1603,1511,1465,1245,1173,1030 \mathrm{~cm}^{-1} ; \mathrm{HRMS}\left[\mathrm{C}_{15} \mathrm{H}_{18} \mathrm{NO}_{2}\right]^{+}$ calcd 244.1338. Found 244.1337 (FAB+).

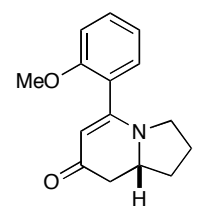

(R)-5-(2-methoxyphenyl)-2,3,8,8a-tetrahydroindolizin-7(1H)-one (4d). According to the general procedure with ligand L5, alkyne 1d, and isocyanate 2. Upon purification $42 \mathrm{mg}(64 \%)$ of the desired product was isolated: $\mathrm{R}_{\mathrm{f}}=0.11\left(\right.$ EtOAc); $[\alpha]_{\mathrm{D}}{ }^{20}=+464.8\left(\mathrm{c}=1.0, \mathrm{CHCl}_{3}\right)$; HPLC analysis - Chiracel AD-H column 85:15 hexane:iPrOH, $1.0 \mathrm{ml} / \mathrm{min}$, Major: 14.5 minutes, Minor: 13.9 minutes, $330 \mathrm{~nm}$ detection light, ee $=94 \%$; ${ }^{1} \mathrm{H}$ NMR (400 $\left.\mathrm{MHz}, \mathrm{CDCl}_{3}\right) \delta 7.36(\mathrm{ddd}, 1 \mathrm{H}, J=1.5,8.5,8.5 \mathrm{~Hz}), 7.18(\mathrm{~m}, 1 \mathrm{H}), 6.96$ (dd, $1 \mathrm{H}, J=7.5$, $7.5 \mathrm{~Hz}), 6.90(\mathrm{~d}, 1 \mathrm{H}, J=8.3 \mathrm{~Hz}), 4.95(\mathrm{~s}, 1 \mathrm{H}), 3.95(\mathrm{~m}, 1 \mathrm{H}), 3.81(\mathrm{~s}, 3 \mathrm{H}), 3.10-3.35(\mathrm{~m}$, $2 \mathrm{H}), 2.39-2.48(\mathrm{~m}, 2 \mathrm{H}), 2.28(\mathrm{dddd}, 1 \mathrm{H}, J=2.8,6.4,6.4,8.7 \mathrm{~Hz}), 1.96(\mathrm{~m}, 1 \mathrm{H}), 1.68-$ $1.88(\mathrm{~m}, 2 \mathrm{H}) ;{ }^{13} \mathrm{C}$ NMR $\left(100 \mathrm{MHz}, \mathrm{CDCl}_{3}\right) \delta 191.8,160.8,155.9,131.2,129.5,125.4$, 121.0, 111.2, 99.4, 59.2, 55.9, 48.1, 41.7, 32.8, 24.2; IR ( $\left.\mathrm{NaCl}, \mathrm{CDCl}_{3}\right)$ 1619, 1527, 1475 , 1332, 1240, 1132, $1015 \mathrm{~cm}^{-1}$; HRMS $\left[\mathrm{C}_{15} \mathrm{H}_{18} \mathrm{NO}_{2}\right]^{+}$calcd 244.1338. Found 244.1336 $(\mathrm{FAB}+)$.

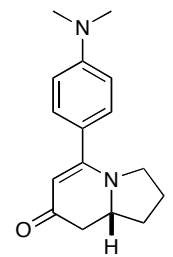

(R)-5-(4-(dimethylamino)phenyl)-2,3,8,8a-tetrahydroindolizin-7(1H)-one (4e). According to the general procedure with ligand L3, alkyne 1e, and isocyanate 2. Upon purification $52.5 \mathrm{mg}(78 \%)$ of the desired product was isolated: $\mathrm{R}_{\mathrm{f}}=0.05\left(\right.$ EtOAc); $[\alpha]_{\mathrm{D}}{ }^{20}=+70.3\left(\mathrm{c}=1.0, \mathrm{CHCl}_{3}\right)$; HPLC analysis - Chiracel OD-H column 85:15 hexane:iPrOH, $1.0 \mathrm{ml} / \mathrm{min}$, Major: 21.2 minutes, Minor: 24.4 minutes, $330 \mathrm{~nm}$ detection light, ee $=87 \%$; ${ }^{1} \mathrm{H}$ NMR $\left(400 \mathrm{MHz}, \mathrm{CDCl}_{3}\right) \delta 7.28(\mathrm{~d}, 2 \mathrm{H}, J=9.0 \mathrm{~Hz}), 6.65(\mathrm{~d}, 2 \mathrm{H}, J=9.0 \mathrm{~Hz}), 5.11(\mathrm{~s}, 1 \mathrm{H}), 4.04$ (dddd, 1H, $J=6.4,6.4,6.4,12.4 \mathrm{~Hz}$ ), 3.60 (ddd, 1H, $J=6.6,6.6,10.9 \mathrm{~Hz}$ ), 3.31 (ddd, $1 \mathrm{H}, J=6.6,6.6,11.1 \mathrm{~Hz}), 2.98(\mathrm{~s}, 6 \mathrm{H}), 2.45(\mathrm{dd}, 1 \mathrm{H}, J=16.0,16.0 \mathrm{~Hz}), 2.34(\mathrm{dd}, 1 \mathrm{H}, J$ $=4.9,16.0 \mathrm{~Hz}), 2.25(\mathrm{dddd}, 1 \mathrm{H}, J=6.8,6.8,6.8,13.4 \mathrm{~Hz}), 1.96(\mathrm{~m}, 1 \mathrm{H}), 1.86(\mathrm{~m}, 1 \mathrm{H})$, 1.73 (dddd, $1 \mathrm{H}, J=6.9,6.9,6.9,13.4 \mathrm{~Hz}) ;{ }^{13} \mathrm{C} \mathrm{NMR}\left(100 \mathrm{MHz}, \mathrm{CDCl}_{3}\right) \delta 191.9,163.8$, $151.7,129.5,123.4,111.5,99.3,58.7,50.3,41.7,40.4,31.6,25.0 ; \mathrm{IR}\left(\mathrm{NaCl}, \mathrm{CDCl}_{3}\right)$ 1603, 1557, 1496, 1358, 1240, 1189, $1132 \mathrm{~cm}^{-1}$; HRMS $\left[\mathrm{C}_{16} \mathrm{H}_{21} \mathrm{~N}_{2} \mathrm{O}\right]^{+}$calcd 257.1654. Found 257.1643 (FAB+).

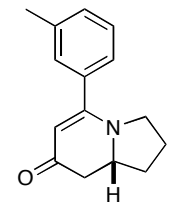

(R)-5-m-tolyl-2,3,8,8a-tetrahydroindolizin-7(1H)-one (4f). According to the general procedure with ligand L5, alkyne 1f, and isocyanate 2 . Upon purification $36.1 \mathrm{mg}(59 \%)$ of the desired product was isolated: $\mathrm{R}_{\mathrm{f}}=0.14$ $\left(\right.$ EtOAc); $[\alpha]_{\mathrm{D}}{ }^{20}=+609.2\left(\mathrm{c}=1.0, \mathrm{CHCl}_{3}\right)$; HPLC analysis - Chiracel AD-H column 95:5 hexane:iPrOH, $1.0 \mathrm{ml} / \mathrm{min}$, Major: 22.1 minutes, Minor: 21.9 minutes, $330 \mathrm{~nm}$ detection light, ee $=94 \% ;{ }^{1} \mathrm{H}$ NMR $\left(400 \mathrm{MHz}, \mathrm{CDCl}_{3}\right) \delta 7.26(\mathrm{dd}, 1 \mathrm{H}, J$ $=7.5,7.5 \mathrm{~Hz}), 7.20(\mathrm{~d}, 1 \mathrm{H}, J=7.6 \mathrm{~Hz}), 7.17(\mathrm{~s}, 1 \mathrm{H}), 7.14(\mathrm{~d}, 1 \mathrm{H}, J=7.5 \mathrm{~Hz}), 5.04(\mathrm{~s}$, $1 \mathrm{H}), 3.99$ (dddd, $1 \mathrm{H}, J=7.5,7.5,7.5,14.7 \mathrm{~Hz}), 3.47$ (ddd, $1 \mathrm{H}, J=4.5,7.7,11.5 \mathrm{~Hz}$ ), $3.23(\mathrm{ddd}, 1 \mathrm{H}, J=7.5,7.5,10.9 \mathrm{~Hz}), 2.44(\mathrm{dd}, 1 \mathrm{H}, J=16.2,16.2 \mathrm{~Hz}), 2.39(\mathrm{dd}, 1 \mathrm{H}, J=$ 7.0, 16.2 Hz), 2.28 (dddd, 1H, $J=4.1,6.4,6.4,10.9 \mathrm{~Hz}), 1.98(\mathrm{~m}, 1 \mathrm{H}), 1.86(\mathrm{~m}, 1 \mathrm{H})$, $1.74(\mathrm{~m}, 1 \mathrm{H}) ;{ }^{13} \mathrm{C}$ NMR $\left(100 \mathrm{MHz}, \mathrm{CDCl}_{3}\right) \delta 192.1,163.3,138.5,136.4,130.7,128.6$, 128.4, 124.8, 100.1, 59.1, 49.5, 41.6, 32.2, 24.7, 21.6; IR $\left(\mathrm{NaCl}, \mathrm{CDCl}_{3}\right)$ 1624, 1527, $1470,1337,1260,1235,1127 \mathrm{~cm}^{-1}$; HRMS $\left[\mathrm{C}_{15} \mathrm{H}_{18} \mathrm{NO}\right]^{+}$calcd 228.1388. Found 228.1384 $(\mathrm{FAB}+)$. 
From the same reaction mixture, $4 \mathrm{mg}(6 \%)$ of $\mathbf{3 f}$ was also isolated but not fully characterized due to its small quantity.

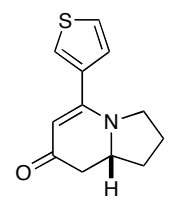

(R)-5-(thiophen-3-yl)-2,3,8,8a-tetrahydroindolizin-7(1H)-one

(4g). According to the general procedure with ligand $\mathbf{L 3}$, alkyne $\mathbf{1 g}$, and isocyanate 2. Upon purification $34.2 \mathrm{mg}(58 \%)$ of the desired product was isolated: $\mathrm{R}_{\mathrm{f}}=$ $0.10\left(\right.$ EtOAc); $[\alpha]_{\mathrm{D}}{ }^{20}=+455.0\left(\mathrm{c}=1.0, \mathrm{CHCl}_{3}\right)$; HPLC analysis - Chiracel AD-H column 80:20 hexane:iPrOH, $1.0 \mathrm{ml} / \mathrm{min}$, Major: 10.6 minutes, Minor: 10.1 minutes, $330 \mathrm{~nm}$ detection light, ee $=86 \% ;{ }^{1} \mathrm{H} \mathrm{NMR}\left(400 \mathrm{MHz}, \mathrm{CDCl}_{3}\right) \delta 7.47$ (dd, $1 \mathrm{H}, J=1.1,3.0 \mathrm{~Hz}$ ), $7.33(\mathrm{dd}, 1 \mathrm{H}, J=3.0,5.1 \mathrm{~Hz}), 7.13(\mathrm{dd}, 1 \mathrm{H}, J=1.3,5.1 \mathrm{~Hz}), 5.15$ (s, 1H), 3.99 (dddd, $1 \mathrm{H}, J=7.5,7.5,7.5,14.5 \mathrm{~Hz}$ ), 3.59 (ddd, $1 \mathrm{H}, J=5.1,7.7,11.1 \mathrm{~Hz}$ ), 3.39 (ddd, $1 \mathrm{H}, J=7.2,7.2,10.9 \mathrm{~Hz}), 2.43(\mathrm{dd}, 1 \mathrm{H}, J=16.2,16.2 \mathrm{~Hz}), 2.39(\mathrm{dd}, 1 \mathrm{H}, J=$ 7.0, 16.2 Hz), 2.28 (dddd, $1 \mathrm{H}, J=6.6,6.6,6.6,11.9 \mathrm{~Hz}), 2.02(\mathrm{~m}, 1 \mathrm{H}), 1.89(\mathrm{~m}, 1 \mathrm{H})$, $1.75(\mathrm{~m}, 1 \mathrm{H}) ;{ }^{13} \mathrm{C}$ NMR $\left(100 \mathrm{MHz}, \mathrm{CDCl}_{3}\right) \delta 192.2,157.6,137.1,127.3,126.5,126.5$, 99.8, 59.1, 49.8, 41.6, 31.9, 24.8; IR ( $\left.\mathrm{NaCl}, \mathrm{CDCl}_{3}\right)$ 1619, 1521, 1470, 1332, 1265, 1240, $1127 \mathrm{~cm}^{-1}$; HRMS $\left[\mathrm{C}_{12} \mathrm{H}_{14} \mathrm{NOS}\right]^{+}$calcd 220.0796. Found 220.0797 (FAB+).

From the same reaction mixture, $3.8 \mathrm{mg}(6 \%)$ of $\mathbf{3 g}$ was also isolated but not fully characterized due to its small quantity.

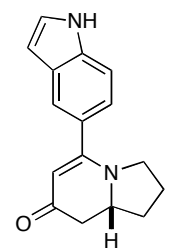

(R)-5-(1H-indol-5-yl)-2,3,8,8a-tetrahydroindolizin-7(1H)-one

(4h). According to the general procedure with ligand L5, alkyne $\mathbf{1 h}$, and isocyanate 2. Upon purification $44.3 \mathrm{mg}(65 \%)$ of the desired product was isolated: $\mathrm{R}_{\mathrm{f}}=$ $0.05\left(\right.$ EtOAc); $[\alpha]_{\mathrm{D}}{ }^{20}=+290.2\left(\mathrm{c}=1.0, \mathrm{CHCl}_{3}\right)$; HPLC analysis - Chiracel AD-H column 80:20 hexane:iPrOH, $1.0 \mathrm{ml} / \mathrm{min}$, Major: 12.9 minutes, Minor: 15.5 minutes, $254 \mathrm{~nm}$ detection light, $e e=90 \% ;{ }^{1} \mathrm{H} \mathrm{NMR}\left(400 \mathrm{MHz}, \mathrm{CDCl}_{3}\right.$ ) $\delta 8.91($ br s, $1 \mathrm{H}), 7.67(\mathrm{~s}, 1 \mathrm{H}), 7.40(\mathrm{~d}, 1 \mathrm{H}, J=8.3 \mathrm{~Hz}), 7.26(\mathrm{dd}, 1 \mathrm{H}, J=2.7,2.7 \mathrm{~Hz})$, $7.16(\mathrm{~d}, 1 \mathrm{H}, J=8.3 \mathrm{~Hz}), 6.55(\mathrm{~m}, 1 \mathrm{H}), 5.19(\mathrm{~s}, 1 \mathrm{H}), 4.08$ (dddd, 1H, $J=6.8,6.8,6.8$, $13.0 \mathrm{~Hz}), 3.61(\mathrm{ddd}, 1 \mathrm{H}, J=6.4,6.4,11.7 \mathrm{~Hz}), 3.29(\mathrm{ddd}, 1 \mathrm{H}, J=7.0,7.0,11.1 \mathrm{~Hz}), 2.51$ (dd, $1 \mathrm{H}, J=16.0,16.0 \mathrm{~Hz}), 2.42(\mathrm{dd}, 1 \mathrm{H}, J=5.5,16.2 \mathrm{~Hz}), 2.30$ (dddd, $1 \mathrm{H}, J=6.2,6.2$, 6.2, $12.2 \mathrm{~Hz}), 1.97(\mathrm{~m}, 1 \mathrm{H}), 1.88(\mathrm{~m}, 1 \mathrm{H}), 1.76(\mathrm{~m}, 1 \mathrm{H}) ;{ }^{13} \mathrm{C} \mathrm{NMR}\left(100 \mathrm{MHz}, \mathrm{CDCl}_{3}\right) \delta$ $191.7,165.3$, 136.8, 127.8, 127.7, 125.9, 121.8, 120.8, 111.4, 103.2, 100.1, 59.0, 50.1, 41.5, 31.9, 24.8; IR ( NaCl, $\left.\mathrm{CDCl}_{3}\right)$ 1598, 1506, 1455, 1334, 1271, 1237, $1128 \mathrm{~cm}^{-1}$; HRMS $\left[\mathrm{C}_{16} \mathrm{H}_{17} \mathrm{~N}_{2} \mathrm{O}\right]^{+}$calcd 253.1341. Found 253.1344 (FAB+).

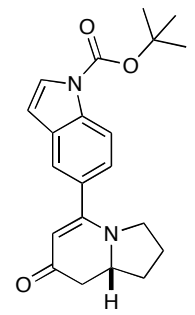

(R)-tert-butyl 5-(7-oxo-1,2,3,7,8,8a-hexahydroindolizin-5-yl)-1H-indole1-carboxylate (4i). According to the general procedure with ligand L5, alkyne 1i, and isocyanate 2 . Upon purification $80.6 \mathrm{mg}(85 \%)$ of the desired product was isolated: $\mathrm{R}_{\mathrm{f}}=0.12\left(\right.$ EtOAc); $[\alpha]_{\mathrm{D}}{ }^{20}=+320.4\left(\mathrm{c}=1.0, \mathrm{CHCl}_{3}\right)$; HPLC analysis - Chiracel OD-H column 90:10 hexane:iPrOH, $1.0 \mathrm{ml} / \mathrm{min}$, Major: 24.9 minutes, Minor: 22.4 minutes, $230 \mathrm{~nm}$ detection light, ee = 91\%; ${ }^{1} \mathrm{H}$ NMR $\left(400 \mathrm{MHz}, \mathrm{CDCl}_{3}\right) \delta 8.16(\mathrm{~d}, 1 \mathrm{H}, J=8.3 \mathrm{~Hz}), 7.62(\mathrm{~d}, 1 \mathrm{H}, J$ $=3.6 \mathrm{~Hz}), 7.58(\mathrm{~s}, 1 \mathrm{H}), 7.30(\mathrm{~d}, 1 \mathrm{H}, J=8.5 \mathrm{~Hz}), 6.55(\mathrm{~d}, 1 \mathrm{H}, J=3.6), 5.15(\mathrm{~s}, 1 \mathrm{H}), 4.05$ (dddd, $1 \mathrm{H}, J=6.8,6.8,6.8,13.9 \mathrm{~Hz}$ ), 3.54 (ddd, $1 \mathrm{H}, J=5.1,7.5,11.7 \mathrm{~Hz}$ ), 3.25 (ddd, $1 \mathrm{H}, J=7.3,7.3,11.1 \mathrm{~Hz}), 2.48(\mathrm{dd}, 1 \mathrm{H}, J=16.0,16.0 \mathrm{~Hz}), 2.42(\mathrm{dd}, 1 \mathrm{H}, J=6.1,16.2$ $\mathrm{Hz}$ ), 2.30 (dddd, 1H, $J=6.4,6.4,6.4,11.5 \mathrm{~Hz}), 1.99(\mathrm{~m}, 1 \mathrm{H}), 1.88(\mathrm{~m}, 1 \mathrm{H}), 1.77(\mathrm{~m}$, 1H), $1.65(\mathrm{~s}, 9 \mathrm{H}) ;{ }^{13} \mathrm{C}$ NMR $\left(100 \mathrm{MHz}, \mathrm{CDCl}_{3}\right) \delta 192.0,163.8,149.7,136.0,130.9$, 
130.6, 127.3, 124.0, 120.7, 115.3, 107.5, 100.4, 84.4, 59.1, 49.7, 41.7, 32.1, 28.3, 24.8; IR $\left(\mathrm{NaCl}, \mathrm{CDCl}_{3}\right) 1736,1615,1524,1449,1369,1329,1271,1151,1025 \mathrm{~cm}^{-1}$; HRMS $\left[\mathrm{C}_{21} \mathrm{H}_{25} \mathrm{~N}_{2} \mathrm{O}_{3}\right]^{+}$calcd 353.1865. Found 353.1852 (FAB+).

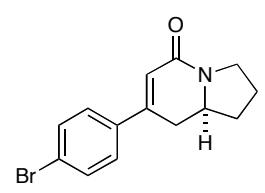

(S)-7-(4-bromophenyl)-2,3,8,8a-tetrahydroindolizin-5(1H)-one (3j). According to the general procedure with ligand $\mathbf{L 5}$, alkyne $\mathbf{1 j}$, and isocyanate 2. Upon purification $13.5 \mathrm{mg}(17 \%)$ of the desired product was isolated: $\mathrm{R}_{\mathrm{f}}=0.19\left(\right.$ EtOAc); $[\alpha]_{\mathrm{D}}{ }^{20}=+102.0\left(\mathrm{c}=1.0, \mathrm{CHCl}_{3}\right)$; HPLC analysis - Chiracel AD-H column 90:10 hexane:iPrOH, $1.0 \mathrm{ml} / \mathrm{min}$, Major: 23.6 minutes, Minor: 25.0 minutes, $254 \mathrm{~nm}$ detection light, ee $=90 \% ;{ }^{1} \mathrm{H}$ NMR $(400 \mathrm{MHz}$, $\left.\mathrm{CDCl}_{3}\right) \delta 7.49(\mathrm{~d}, 2 \mathrm{H}, J=8.7 \mathrm{~Hz}), 7.33(\mathrm{~d}, 2 \mathrm{H}, J=8.7 \mathrm{~Hz}), 6.27(\mathrm{~d}, 1 \mathrm{H}, J=2.6 \mathrm{~Hz}), 3.80$ (dddd, $1 \mathrm{H}, J=5.1,5.1,10.2,14.9 \mathrm{~Hz}), 3.67(\mathrm{~m}, 1 \mathrm{H}), 3.50(\mathrm{~m}, 1 \mathrm{H}), 2.84(\mathrm{dd}, 1 \mathrm{H}, J=4.7$, $16.4 \mathrm{~Hz}$ ), 2.50 (ddd, $1 \mathrm{H}, J=2.6,14.1,16.6 \mathrm{~Hz}), 2.27$ (ddd, $1 \mathrm{H}, J=5.8,5.8,11.9 \mathrm{~Hz}$ ), $2.06(\mathrm{~m}, 1 \mathrm{H}), 1.84(\mathrm{~m}, 1 \mathrm{H}), 1.71(\mathrm{~m}, 1 \mathrm{H}) ;{ }^{13} \mathrm{C} \mathrm{NMR}\left(100 \mathrm{MHz}, \mathrm{CDCl}_{3}\right) \delta 163.9,147.6$, 136.9, 132.1, 127.6, 123.7, 121.4, 56.7, 44.2, 33.7, 33.3, 23.2; IR ( $\left.\mathrm{NaCl}, \mathrm{CDCl}_{3}\right)$ 1650, 1539, 1486, 1445, 1347, 1004, 835, $815 \mathrm{~cm}^{-1}$; HRMS $\left[\mathrm{C}_{14} \mathrm{H}_{15} \mathrm{NOBr}\right]^{+}$calcd 292.0337. Found 292.0333 (FAB+). X-ray data of this compound is attached at the end of this manuscript.

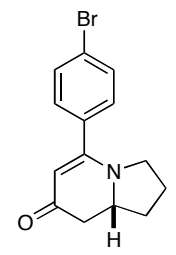

(R)-5-(4-bromophenyl)-2,3,8,8a-tetrahydroindolizin-7(1H)-one

$(4 \mathbf{j})$. According to the general procedure with ligand $\mathbf{L 5}$, alkyne $\mathbf{1 j}$, and isocyanate 2. From the same reaction as above, upon purification $43.4 \mathrm{mg}(55 \%)$ of the desired product was isolated: $\mathrm{R}_{\mathrm{f}}=0.13\left(\right.$ EtOAc); $[\alpha]_{\mathrm{D}}{ }^{20}=+469.0(\mathrm{c}=1.0$, $\mathrm{CHCl}_{3}$ ); HPLC analysis - Chiracel OJ-H column 90:10 hexane:iPrOH, 1.0 $\mathrm{ml} / \mathrm{min}$, Major: 21.3 minutes, Minor: 19.7 minutes, $254 \mathrm{~nm}$ detection light, ee $=89 \% ;{ }^{1} \mathrm{H}$ NMR $\left(400 \mathrm{MHz}, \mathrm{CDCl}_{3}\right) \delta 7.52(\mathrm{~d}, 2 \mathrm{H}, J=8.3 \mathrm{~Hz}), 7.24(\mathrm{~d}, 2 \mathrm{H}, J=8.3 \mathrm{~Hz})$, $5.01(\mathrm{~s}, 1 \mathrm{H}), 4.00$ (dddd, $1 \mathrm{H}, J=7.9,7.9,7.9,15.3 \mathrm{~Hz}), 3.44$ (ddd, $1 \mathrm{H}, J=4.5,7.3,11.3$ $\mathrm{Hz}), 3.22(\mathrm{ddd}, 1 \mathrm{H}, J=7.7,7.7,10.7 \mathrm{~Hz}), 2.44(\mathrm{dd}, 1 \mathrm{H}, J=16.2,16.2 \mathrm{~Hz}), 2.42(\mathrm{dd}, 1 \mathrm{H}$, $J=7.8,16.2 \mathrm{~Hz}), 2.29(\mathrm{~m}, 1 \mathrm{H}), 1.99(\mathrm{~m}, 1 \mathrm{H}), 1.87(\mathrm{~m}, 1 \mathrm{H}), 1.75(\mathrm{~m}, 1 \mathrm{H}) ;{ }^{13} \mathrm{C} \mathrm{NMR}(100$ $\left.\mathrm{MHz}, \mathrm{CDCl}_{3}\right) \delta 192.2,161.8,135.3,132.0,129.4,124.4,100.4,59.2,49.5,41.6,32.1$, 24.7; IR $\left(\mathrm{NaCl}, \mathrm{CDCl}_{3}\right)$ 1619, 1568, 1527, 1465, 1260, 1235, 1127, $1004 \mathrm{~cm}^{-1}$; HRMS $\left[\mathrm{C}_{14} \mathrm{H}_{15} \mathrm{NOBr}\right]^{+}$calcd 292.0337. Found $292.0330(\mathrm{FAB}+)$. X-ray of this compound is attached at the end of this manuscript.

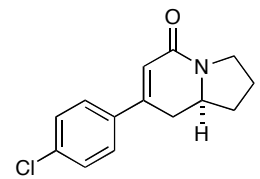

(S)-7-(4-chlorophenyl)-2,3,8,8a-tetrahydroindolizin-5(1H)-one (3k). According to the general procedure with ligand L5, alkyne $\mathbf{1 k}$, and isocyanate 2 . Upon purification $9.4 \mathrm{mg}(14 \%)$ of the desired product was isolated: $\mathrm{R}_{\mathrm{f}}=0.13($ EtOAc $) ;[\alpha]_{\mathrm{D}}{ }^{20}=+93.5\left(\mathrm{c}=0.52, \mathrm{CHCl}_{3}\right)$; HPLC analysis - Chiracel OD-H column 90:10 hexane:iPrOH, $1.0 \mathrm{ml} / \mathrm{min}$, Major: 19.5 minutes, Minor: 18.1 minutes, $254 \mathrm{~nm}$ detection light, ee $=93 \% ;{ }^{1} \mathrm{H}$ NMR $(400 \mathrm{MHz}$, $\left.\mathrm{CDCl}_{3}\right) \delta 7.40(\mathrm{~d}, 2 \mathrm{H}, J=8.5 \mathrm{~Hz}), 7.34(\mathrm{~d}, 2 \mathrm{H}, J=8.5 \mathrm{~Hz}), 6.27(\mathrm{~d}, 1 \mathrm{H}, J=2.8 \mathrm{~Hz}), 3.81$ (dddd, $1 \mathrm{H}, J=5.1,5.1,10.2,14.7 \mathrm{~Hz}$ ), 3.67 (ddd, $1 \mathrm{H}, J=2.1,9.2,11.7 \mathrm{~Hz}$ ), 3.50 (ddd, $1 \mathrm{H}, J=7.7,9.6,11.5 \mathrm{~Hz}), 2.85(\mathrm{dd}, 1 \mathrm{H}, J=4.7,16.4 \mathrm{~Hz}), 2.50$ (ddd, $1 \mathrm{H}, J=2.6,13.9$, $16.4 \mathrm{~Hz}), 2.27(\mathrm{ddd}, 1 \mathrm{H}, J=5.0,5.0,11.3 \mathrm{~Hz}), 2.06(\mathrm{~m}, 1 \mathrm{H}), 1.84(\mathrm{~m}, 1 \mathrm{H}), 1.71(\mathrm{~m}, 1 \mathrm{H})$; ${ }^{13} \mathrm{C}$ NMR $\left(100 \mathrm{MHz}, \mathrm{CDCl}_{3}\right) \delta 163.9,147.5,136.5,135.5,129.2,127.3,121.3,56.7$, 
44.2, 33.7, 33.3, 23.2; IR ( $\left.\mathrm{NaCl}, \mathrm{CDCl}_{3}\right)$ 1650, 1598, 1489, 1443, 1345, 1087, $829 \mathrm{~cm}^{-1}$; HRMS $\left[\mathrm{C}_{14} \mathrm{H}_{15} \mathrm{NOCl}\right]^{+}$calcd 248.0842. Found 248.0853 (FAB+).

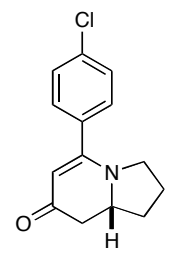

(R)-5-(4-chlorophenyl)-2,3,8,8a-tetrahydroindolizin-7(1H)-one

(4k). According to the general procedure with ligand $\mathbf{L 5}$, alkyne $\mathbf{1 k}$, and isocyanate 2. From the same reaction as above, upon purification $34.1 \mathrm{mg}(51 \%)$ of the desired product was isolated: $\mathrm{R}_{\mathrm{f}}=0.08\left(\right.$ EtOAc); $[\alpha]_{\mathrm{D}}{ }^{20}=+383.9(\mathrm{c}=1.0$, $\mathrm{CHCl}_{3}$ ); HPLC analysis - Chiracel OJ-H column 95:5 hexane:iPrOH, 1.0 $\mathrm{ml} / \mathrm{min}$, Major: 23.3 minutes, Minor: 22.3 minutes, $330 \mathrm{~nm}$ detection light, ee $=90 \% ;{ }^{1} \mathrm{H}$ NMR $\left(400 \mathrm{MHz}, \mathrm{CDCl}_{3}\right) \delta 7.37(\mathrm{~d}, 2 \mathrm{H}, J=8.3 \mathrm{~Hz}), 7.31(\mathrm{~d}, 2 \mathrm{H}, J=8.3 \mathrm{~Hz})$, $5.03(\mathrm{~s}, 1 \mathrm{H}), 4.01$ (dddd, $1 \mathrm{H}, J=7.9,7.9,7.9,15.1 \mathrm{~Hz}), 3.45$ (ddd, $1 \mathrm{H}, J=4.5,7.5,11.7$ $\mathrm{Hz}), 3.23$ (ddd, 1H, $J=7.7,7.7,10.9 \mathrm{~Hz}), 2.45$ (dd, 1H, $J=16.2,16.2 \mathrm{~Hz}), 2.42$ (dd, 1H, $J=8.0,16.2 \mathrm{~Hz}), 2.29(\mathrm{~m}, 1 \mathrm{H}), 1.99(\mathrm{~m}, 1 \mathrm{H}), 1.88(\mathrm{~m}, 1 \mathrm{H}), 1.76(\mathrm{~m}, 1 \mathrm{H}) ;{ }^{13} \mathrm{C} \mathrm{NMR}(100$ $\left.\mathrm{MHz}, \mathrm{CDCl}_{3}\right) \delta 192.2,161.8,136.1,134.8,129.2,129.1,100.4,59.2$, 49.5, 41.6, 32.1, 24.7; IR $\left(\mathrm{NaCl}, \mathrm{CDCl}_{3}\right)$ 1624, 1572, 1527, 1470, 1265, 1240, 1086, $1004 \mathrm{~cm}^{-1}$; HRMS $\left[\mathrm{C}_{14} \mathrm{H}_{15} \mathrm{NOCl}\right]^{+}$calcd 248.0842. Found $248.0843(\mathrm{FAB}+)$.

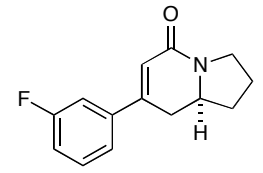

(S)-7-(3-fluorophenyl)-2,3,8,8a-tetrahydroindolizin-5(1H)-one $\quad(3 \mathrm{l})$. According to the general procedure with ligand L5, alkyne 11, and isocyanate 2. Upon purification $15.5 \mathrm{mg}(25 \%)$ of the desired product was isolated: $\mathrm{R}_{\mathrm{f}}=0.21($ EtOAc $) ;[\alpha]_{\mathrm{D}}{ }^{20}=+88.3\left(\mathrm{c}=1.0, \mathrm{CHCl}_{3}\right) ; \mathrm{HPLC}$ analysis - Chiracel AD-H column 90:10 hexane:iPrOH, $1.0 \mathrm{ml} / \mathrm{min}$, Major: 17.1 minutes, Minor: 12.9 minutes, $254 \mathrm{~nm}$ detection light, ee $=94 \% ;{ }^{1} \mathrm{H} \mathrm{NMR}\left(400 \mathrm{MHz}, \mathrm{CDCl}_{3}\right) \delta$ 7.33 (ddd, $1 \mathrm{H}, J=6.0,8.1,8.1 \mathrm{~Hz}), 7.25(\mathrm{~m}, 1 \mathrm{H}), 7.15(\mathrm{ddd}, 1 \mathrm{H}, J=2.1,2.1,10.2 \mathrm{~Hz})$, $7.04(\mathrm{~m}, 1 \mathrm{H}), 6.29(\mathrm{~d}, 1 \mathrm{H}, J=2.8 \mathrm{~Hz}), 3.81$ (dddd, $1 \mathrm{H}, J=5.1,5.1,10.2,14.9 \mathrm{~Hz}), 3.67$ $(\mathrm{ddd}, 1 \mathrm{H}, J=1.7,8.7,11.5 \mathrm{~Hz}), 3.50(\mathrm{ddd}, 1 \mathrm{H}, J=7.7,9.4,11.9 \mathrm{~Hz}), 2.85(\mathrm{dd}, 1 \mathrm{H}, J=$ $4.7,16.6 \mathrm{~Hz}), 2.51(\mathrm{ddd}, 1 \mathrm{H}, J=2.8,14.1,16.6 \mathrm{~Hz}), 2.27(\mathrm{ddd}, 1 \mathrm{H}, J=5.5,5.5,11.9$ $\mathrm{Hz}), 2.07(\mathrm{~m}, 1 \mathrm{H}), 1.84(\mathrm{~m}, 1 \mathrm{H}), 1.70(\mathrm{~m}, 1 \mathrm{H}) ;{ }^{13} \mathrm{C} \mathrm{NMR}\left(100 \mathrm{MHz}, \mathrm{CDCl}_{3}\right) \delta 164.4$, $163.8,161.9,147.4,130.5,130.4,121.9,121.7,116.4,116.2,113.1,112.9,56.7,44.2$, 33.7, 33.4, 23.2; IR ( $\left.\mathrm{NaCl}, \mathrm{CDCl}_{3}\right)$ 1650, 1598, 1434, 1327, 1265, 1173, 1158, $876 \mathrm{~cm}^{-1}$; HRMS $\left[\mathrm{C}_{14} \mathrm{H}_{15} \mathrm{NOF}\right]^{+}$calcd 232.1138. Found 232.1141 (FAB+).

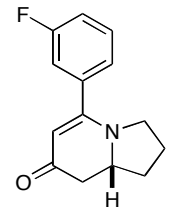

(R)-5-(3-fluorophenyl)-2,3,8,8a-tetrahydroindolizin-7(1H)-one

(4l). According to the general procedure with ligand L5, alkyne 11, and isocyanate 2. From the same reaction as above, upon purification $27.0 \mathrm{mg}(43 \%)$ of the desired product was isolated: $\mathrm{R}_{\mathrm{f}}=0.15\left(\right.$ EtOAc); $[\alpha]_{\mathrm{D}}{ }^{20}=+366.7(\mathrm{c}=1.0$, $\mathrm{CHCl}_{3}$ ); HPLC analysis - Chiracel OJ-H column 97:3 hexane:iPrOH, 1.0 $\mathrm{ml} / \mathrm{min}$, Major: 25.6 minutes, Minor: 24.8 minutes, $330 \mathrm{~nm}$ detection light, $e e=94 \% ;{ }^{1} \mathrm{H}$ NMR (400 MHz, $\left.\mathrm{CDCl}_{3}\right) \delta 7.36(\mathrm{ddd}, 1 \mathrm{H}, J=5.8,7.9,7.9 \mathrm{~Hz}), 7.05-7.16(\mathrm{~m}, 3 \mathrm{H}), 5.05$ (s, 1H), 4.00 (dddd, $1 \mathrm{H}, J=8.8,8.1,8.1,12.2 \mathrm{~Hz}$ ), 3.45 (ddd, $1 \mathrm{H}, J=4.5,7.7,11.3 \mathrm{~Hz}$ ), 3.24 (ddd, 1H, $J=7.5,7.5,10.7 \mathrm{~Hz}), 2.46(\mathrm{dd}, 1 \mathrm{H}, J=16.2,16.2 \mathrm{~Hz}), 2.42(\mathrm{dd}, 1 \mathrm{H}, J=$ 7.5, 16.2 Hz), 2.30 (dddd, $1 \mathrm{H}, J=4.1,6.4,6.4,10.9 \mathrm{~Hz}), 2.01(\mathrm{~m}, 1 \mathrm{H}), 1.88(\mathrm{~m}, 1 \mathrm{H})$, $1.75(\mathrm{~m}, 1 \mathrm{H}) ;{ }^{13} \mathrm{C}$ NMR $\left(100 \mathrm{MHz}, \mathrm{CDCl}_{3}\right) \delta 192.2,163.9,161.6,161.4,138.4,130.6$, $130.5,123.5,117.1,116.9,115.1,114.9,100.4,59.2,49.5,41.6,32.2,24.7$; IR $(\mathrm{NaCl}$, $\left.\mathrm{CDCl}_{3}\right) 1629,1527,1470,1260,1239,1122,933 \mathrm{~cm}^{-1}$; HRMS $\left[\mathrm{C}_{14} \mathrm{H}_{15} \mathrm{NOF}\right]^{+}$calcd 232.1138. Found 232.1139 (FAB+). 


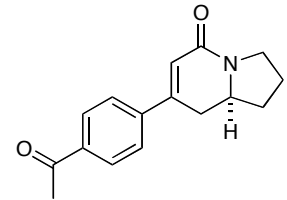

(S)-7-(4-acetylphenyl)-2,3,8,8a-tetrahydroindolizin-5(1H)-one (3m). According to the general procedure with ligand L5, alkyne $\mathbf{1 m}$, and isocyanate 2 . Upon purification $17.8 \mathrm{mg}(26 \%)$ of the desired product was isolated: $\mathrm{R}_{\mathrm{f}}=0.07\left(\right.$ EtOAc); $[\alpha]_{\mathrm{D}}{ }^{20}=+82.6(\mathrm{c}=1.0$, $\left.\mathrm{CHCl}_{3}\right) ; \quad$ HPLC analysis - Chiracel AD-H column 80:20 hexane:iPrOH, $1.0 \mathrm{ml} / \mathrm{min}$, Major: 23.0 minutes, Minor: 21.1 minutes, $254 \mathrm{~nm}$ detection light, ee $=94 \% ;{ }^{1} \mathrm{H}$ NMR $\left(400 \mathrm{MHz}, \mathrm{CDCl}_{3}\right) \delta 7.94(\mathrm{~d}, 2 \mathrm{H}, J=8.3 \mathrm{~Hz}), 7.55(\mathrm{~d}, 2 \mathrm{H}, J=$ $8.3 \mathrm{~Hz}$ ), 6.36 (d, 1H, $J=2.8 \mathrm{~Hz}$ ), 3.83 (dddd, 1H, $J=5.1,5.1,10.0,14.7 \mathrm{~Hz}$ ), 3.68 (ddd, $1 \mathrm{H}, J=2.3,9.4,11.7 \mathrm{~Hz}), 3.51(\mathrm{ddd}, 1 \mathrm{H}, J=8.1,9.6,11.8 \mathrm{~Hz}), 2.90(\mathrm{dd}, 1 \mathrm{H}, J=4.7$, $16.6 \mathrm{~Hz}), 2.59(\mathrm{~s}, 3 \mathrm{H}), 2.57(\mathrm{~m}, 1 \mathrm{H}), 2.29(\mathrm{~m}, 1 \mathrm{H}), 2.07(\mathrm{~m}, 1 \mathrm{H}), 1.84(\mathrm{~m}, 1 \mathrm{H}), 1.72(\mathrm{~m}$, $1 \mathrm{H}) ;{ }^{13} \mathrm{C} \mathrm{NMR}\left(100 \mathrm{MHz}, \mathrm{CDCl}_{3}\right) \delta 197.6,163.6,147.5,142.6,137.5,129.0,126.2$, 122.8, 56.7, 44.2, 33.7, 33.3, 26.9, 23.2; IR ( $\left.\mathrm{NaCl}, \mathrm{CDCl}_{3}\right)$ 1672, 1649, 1598, 1445, 1360, $1269,843 \mathrm{~cm}^{-1}$; HRMS $\left[\mathrm{C}_{16} \mathrm{H}_{18} \mathrm{NO}_{2}\right]^{+}$calcd 256.1338. Found 256.1327 (FAB+).

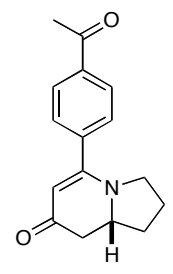

(R)-5-(4-acetylphenyl)-2,3,8,8a-tetrahydroindolizin-7(1H)-one

$(4 \mathrm{~m})$. According to the general procedure with ligand L5, alkyne $\mathbf{1 m}$, and isocyanate 2. From the same reaction as above, upon purification $26.8 \mathrm{mg}$ $(39 \%)$ of the desired product was isolated: $\mathrm{R}_{\mathrm{f}}=0.04\left(\right.$ EtOAc); $[\alpha]_{\mathrm{D}}{ }^{20}=$ $+347.3\left(\mathrm{c}=1.0, \mathrm{CHCl}_{3}\right)$; HPLC analysis - Chiracel OD-H column 80:20 hexane:iPrOH, $1.0 \mathrm{ml} / \mathrm{min}$, Major: 21.3 minutes, Minor: 23.1 minutes, 254 nm detection light, $c a . e e=81 \% ;{ }^{1} \mathrm{H} \mathrm{NMR}\left(400 \mathrm{MHz}, \mathrm{CDCl}_{3}\right) \delta 7.97$ (d, 2H, J=8.3 Hz), $7.46(\mathrm{~d}, 2 \mathrm{H}, J=8.1 \mathrm{~Hz}), 5.02(\mathrm{~s}, 1 \mathrm{H}), 4.01(\mathrm{~m}, 1 \mathrm{H}), 3.42(\mathrm{ddd}, 1 \mathrm{H}, J=4.3,7.7,11.3 \mathrm{~Hz})$, 3.23 (ddd, 1H, $J=7.7,7.7,10.9 \mathrm{~Hz}), 2.60(\mathrm{~s}, 3 \mathrm{H}), 2.42-2.51(\mathrm{~m}, 2 \mathrm{H}), 2.31(\mathrm{~m}, 1 \mathrm{H})$, $2.01(\mathrm{~m}, 1 \mathrm{H}), 1.88(\mathrm{~m}, 1 \mathrm{H}), 1.77(\mathrm{~m}, 1 \mathrm{H}) ;{ }^{13} \mathrm{C} \mathrm{NMR}\left(100 \mathrm{MHz}, \mathrm{CDCl}_{3}\right) \delta 197.5,192.1$, 161.7, 140.7, 138.1, 128.7, 128.1, 100.6, 59.3, 49.4, 41.5, 32.2, 26.9, 24.7; IR (NaCl, $\left.\mathrm{CDCl}_{3}\right)$ 1683, 1621, 1530, 1507, 1468, 1257, $1127 \mathrm{~cm}^{-1}$; HRMS $\left[\mathrm{C}_{16} \mathrm{H}_{18} \mathrm{NO}_{2}\right]^{+}$calcd 256.1338. Found 256.1336 (FAB+).

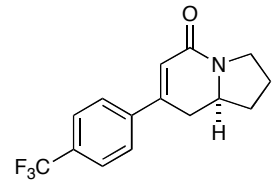

(S)-7-(4-(trifluoromethyl)phenyl)-2,3,8,8a-tetrahydroindolizin5(1H)-one (3n). According to the general procedure with ligand L5, alkyne $\mathbf{1 n}$, and isocyanate 2 . Upon purification $27.2 \mathrm{mg}(36 \%)$ of the desired product was isolated: $\mathrm{R}_{\mathrm{f}}=0.20\left(\right.$ EtOAc); $[\alpha]_{\mathrm{D}}{ }^{20}=+104.5(\mathrm{c}=$ 1.0, $\mathrm{CHCl}_{3}$ ); HPLC analysis - Chiracel OD-H column 90:10 hexane:iPrOH, $1.0 \mathrm{ml} / \mathrm{min}$, Major: 17.0 minutes, Minor: 15.8 minutes, $254 \mathrm{~nm}$ detection light, $e e=94 \%$; ${ }^{1} \mathrm{H} \mathrm{NMR}$ $\left(400 \mathrm{MHz}, \mathrm{CDCl}_{3}\right) \delta 7.63(\mathrm{~d}, 2 \mathrm{H}, J=8.3 \mathrm{~Hz}), 7.56(\mathrm{~d}, 2 \mathrm{H}, J=8.5 \mathrm{~Hz}), 6.34(\mathrm{~d}, 1 \mathrm{H}, J=$ $2.6 \mathrm{~Hz}$ ), 3.83 (dddd, $1 \mathrm{H}, J=5.1,5.1,10.2,14.7 \mathrm{~Hz}$ ), 3.69 (ddd, $1 \mathrm{H}, J=2.1,9.2,11.7$ $\mathrm{Hz}), 3.52$ (ddd, $1 \mathrm{H}, J=7.7,9.6,11.7 \mathrm{~Hz}), 2.88(\mathrm{dd}, 1 \mathrm{H}, J=4.7,16.4 \mathrm{~Hz}), 2.55$ (ddd, $1 \mathrm{H}$, $J=2.6,14.1,16.6 \mathrm{~Hz}), 2.29(\mathrm{~m}, 1 \mathrm{H}), 2.08(\mathrm{~m}, 1 \mathrm{H}), 1.85(\mathrm{~m}, 1 \mathrm{H}), 1.73(\mathrm{~m}, 1 \mathrm{H}) ;{ }^{13} \mathrm{C} \mathrm{NMR}$ $\left(100 \mathrm{MHz}, \mathrm{CDCl}_{3}\right) \delta 163.6,147.3,141.6,126.4,125.9,125.9,122.8,56.7,44.2,33.7$, 33.4, 23.2; IR ( $\left.\mathrm{NaCl}, \mathrm{CDCl}_{3}\right)$ 1654, 1601, 1450, 1410, 1328, 1152, 1124, 1071, $849 \mathrm{~cm}^{-1}$; HRMS $\left[\mathrm{C}_{15} \mathrm{H}_{15} \mathrm{NOF}_{3}\right]^{+}$calcd 282.1106. Found 282.1094 (FAB+).

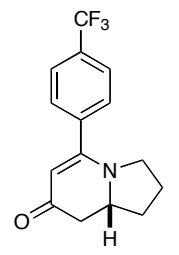

(R)-5-(4-(trifluoromethyl)phenyl)-2,3,8,8a-tetrahydroindolizin-7(1H)one (4n). According to the general procedure with ligand L5, alkyne 1n, and isocyanate 2. From the same reaction as above, upon purification $10.6 \mathrm{mg}$ 
$(14 \%)$ of the desired product was isolated: $\mathrm{R}_{\mathrm{f}}=0.14($ EtOAc $) ;[\alpha]_{\mathrm{D}}{ }^{20}=+318.9(\mathrm{c}=1.0$, $\mathrm{CHCl}_{3}$ ); ee is not determined since the racemate synthesis of $\mathbf{4 n}$ has failed; ${ }^{1} \mathrm{H}$ NMR (400 $\left.\mathrm{MHz}, \mathrm{CDCl}_{3}\right) \delta 7.66(\mathrm{~d}, 2 \mathrm{H}, J=8.1 \mathrm{~Hz}), 7.49(\mathrm{~d}, 2 \mathrm{H}, J=8.1 \mathrm{~Hz}), 5.08(\mathrm{~s}, 1 \mathrm{H}), 4.02$ (dddd, $1 \mathrm{H}, J=9.2,9.2,9.2,9.2 \mathrm{~Hz}$ ), 3.42 (ddd, $1 \mathrm{H}, J=4.1,7.9,11.3 \mathrm{~Hz}$ ), 3.23 (ddd, 1H, $J=7.5,7.5,10.4 \mathrm{~Hz}), 2.42-2.52(\mathrm{~m}, 2 \mathrm{H}), 2.32(\mathrm{~m}, 1 \mathrm{H}), 2.02(\mathrm{~m}, 1 \mathrm{H}), 1.89(\mathrm{~m}, 1 \mathrm{H})$, $1.78(\mathrm{~m}, 1 \mathrm{H}) ;{ }^{13} \mathrm{C} \mathrm{NMR}\left(100 \mathrm{MHz}, \mathrm{CDCl}_{3}\right) \delta 192.0,161.5,150.8,139.8,128.2,125.8$, 100.6, 59.3, 49.4, 41.4, 32.2, 24.7; IR $\left(\mathrm{NaCl}, \mathrm{CDCl}_{3}\right)$ 1625, 1538, 1514, 1461, 1322, $1164,1124,1065 \mathrm{~cm}^{-1}$; HRMS $\left[\mathrm{C}_{15} \mathrm{H}_{15} \mathrm{NOF}_{3}\right]^{+}$calcd 282.1106. Found 282.1115 (FAB+).

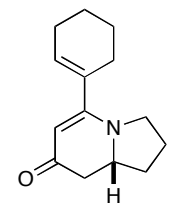

(R)-5-cyclohexenyl-2,3,8,8a-tetrahydroindolizin-7(1H)-one

(4o).

According to the general procedure with ligand L5, alkyne 1o, and isocyanate 2. Upon purification $56.3 \mathrm{mg}(96 \%)$ of the desired product was isolated: $\mathrm{R}_{\mathrm{f}}=$ $0.12\left(\right.$ EtOAc); $[\alpha]_{\mathrm{D}}{ }^{20}=+477.5\left(\mathrm{c}=1.0, \mathrm{CHCl}_{3}\right)$; HPLC analysis - Chiracel AD-H column 85:15 hexane:iPrOH, $1.0 \mathrm{ml} / \mathrm{min}$, Major: 10.9 minutes, Minor: 10.3 minutes, $330 \mathrm{~nm}$ detection light, $e e=92 \%$; ${ }^{1} \mathrm{H}$ NMR $\left(400 \mathrm{MHz}, \mathrm{CDCl}_{3}\right) \delta 5.83(\mathrm{~m}$, $1 \mathrm{H}), 4.88(\mathrm{~s}, 1 \mathrm{H}), 3.80(\mathrm{~m}, 1 \mathrm{H}), 3.46(\mathrm{ddd}, 1 \mathrm{H}, J=4.5,8.1,11.3 \mathrm{~Hz}), 3.34(\mathrm{ddd}, 1 \mathrm{H}, J=$ 7.7, 7.7, $10.9 \mathrm{~Hz}), 2.27-2.35(\mathrm{~m}, 2 \mathrm{H}), 2.21$ (dddd, 1H, J = 4.1, 6.6, 6.6, $12.6 \mathrm{~Hz}), 2.05-$ $2.11(\mathrm{~m}, 4 \mathrm{H}), 2.00(\mathrm{~m}, 1 \mathrm{H}), 1.84(\mathrm{~m}, 1 \mathrm{H}), 1.54-1.72(\mathrm{~m}, 5 \mathrm{H}) ;{ }^{13} \mathrm{C}$ NMR $(100 \mathrm{MHz}$, $\left.\mathrm{CDCl}_{3}\right) \delta 192.4,165.5,134.7,129.7,97.8,58.9,48.6,41.5,32.1,27.4,25.2,24.6,22.5$, 21.9; IR $\left(\mathrm{NaCl}, \mathrm{CDCl}_{3}\right) 1621,1512,1466,1329,1260,1231,1122,922 \mathrm{~cm}^{-1}$; HRMS $\left[\mathrm{C}_{14} \mathrm{H}_{20} \mathrm{NO}\right]^{+}$calcd 218.1545. Found 218.1541 (FAB+).

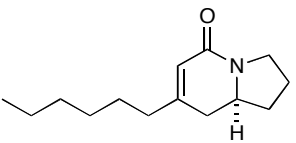

(S)-7-hexyl-2,3,8,8a-tetrahydroindolizin-5(1H)-one

(3p). According to the general procedure with ligand L4, alkyne 1p, and isocyanate 2. Upon purification $38.3 \mathrm{mg}(64 \%)$ of the desired product was isolated: $\mathrm{R}_{\mathrm{f}}=0.39\left(5 \% \mathrm{MeOH} / \mathrm{CH}_{2} \mathrm{Cl}_{2}\right) ;[\alpha]_{\mathrm{D}}{ }^{20}=+95.3\left(\mathrm{c}=1.0, \mathrm{CHCl}_{3}\right)$; HPLC analysis - Chiracel OD-H column 97:3 hexane:iPrOH, $1.0 \mathrm{ml} / \mathrm{min}$, Major: 20.2 minutes, Minor: 21.9 minutes, $230 \mathrm{~nm}$ detection light, ee $=80 \% ;{ }^{1} \mathrm{H} \mathrm{NMR}\left(400 \mathrm{MHz}, \mathrm{CDCl}_{3}\right) \delta$ $5.68(\mathrm{~m}, 1 \mathrm{H}), 3.62(\mathrm{dddd}, 1 \mathrm{H}, J=5.1,5.1,10.2,15.1 \mathrm{~Hz}), 3.58(\mathrm{ddd}, 1 \mathrm{H}, J=2.1,9.4$, $11.7 \mathrm{~Hz}$ ), 3.42 (ddd, 1H, $J=7.9,9.8,11.7 \mathrm{~Hz}), 2.27(\mathrm{dd}, 1 \mathrm{H}, J=4.9,16.6 \mathrm{~Hz}), 2.06-$ $2.19(\mathrm{~m}, 4 \mathrm{H}), 1.98(\mathrm{~m}, 1 \mathrm{H}), 1.75$ (ddddd, $1 \mathrm{H}, J=6.6,9.6,9.6,12.2,12.2 \mathrm{~Hz}), 1.56(\mathrm{~m}$, $1 \mathrm{H}), 1.38-1.46(\mathrm{~m}, 2 \mathrm{H}), 1.21-1.31(\mathrm{~m}, 6 \mathrm{H}), 0.84(\mathrm{t}, 3 \mathrm{H}, J=6.7 \mathrm{~Hz}) ;{ }^{13} \mathrm{C}$ NMR $(100$ $\left.\mathrm{MHz} \mathrm{CDCl}_{3}\right) \delta 164.4,153.6,120.4,56.7,44.0,36.7,34.8,33.7,31.8,29.0,26.9,23.2$, 22.7, 14.3; IR ( $\left.\mathrm{NaCl}, \mathrm{CH}_{2} \mathrm{Cl}_{2}\right) 1660,1609,1445,1342,1265 \mathrm{~cm}^{-1}$; HRMS $\left[\mathrm{C}_{14} \mathrm{H}_{24} \mathrm{NO}^{+}\right.$ calcd 222.1858. Found 222.1853 (FAB+).

From the same reaction mixture, the minor product $4 \mathrm{p}(8.5 \mathrm{mg}, 14 \%)$ was also isolated, and its characterization data is available upon request.

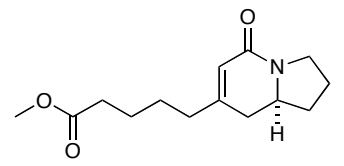

(S)-methyl

5-(5-oxo-1,2,3,5,8,8a-hexahydroindolizin-7yl)pentanoate (3q). According to the general procedure with ligand L4, alkyne 1q, and isocyanate 2. Upon purification $37.8 \mathrm{mg}(56 \%)$ of the desired product was isolated: $\mathrm{R}_{\mathrm{f}}=0.12(\mathrm{EtOAc}) ;[\alpha]_{\mathrm{D}}{ }^{20}=$ +71.6 (c = 1.0, $\left.\mathrm{CHCl}_{3}\right)$; HPLC analysis - Chiracel AD-H column 90:10 hexane:iPrOH, $1.0 \mathrm{ml} / \mathrm{min}$, Major: 16.3 minutes, Minor: 14.4 minutes, $254 \mathrm{~nm}$ detection light, ee $=80 \%$; ${ }^{1} \mathrm{H}$ NMR $\left(400 \mathrm{MHz}, \mathrm{CDCl}_{3}\right) \delta 5.70(\mathrm{~m}, 1 \mathrm{H}), 3.64$ (dddd, $1 \mathrm{H}, J=5.3,5.3,10.4,15.3 \mathrm{~Hz}$ ), 
$3.63(\mathrm{~s}, 3 \mathrm{H}), 3.58(\mathrm{ddd}, 1 \mathrm{H}, J=2.3,9.4,11.7 \mathrm{~Hz}), 3.41(\mathrm{ddd}, 1 \mathrm{H}, J=7.9,9.8,11.7 \mathrm{~Hz})$, $2.29(\mathrm{t}, 2 \mathrm{H}, J=7.3 \mathrm{~Hz}), 2.26(\mathrm{dd}, 1 \mathrm{H}, J=4.9,16.6 \mathrm{~Hz}), 2.06-2.20(\mathrm{~m}, 4 \mathrm{H}), 1.98(\mathrm{~m}$, 1H), 1.76 (ddddd, $1 \mathrm{H}, J=6.6,9.4,9.4,12.2,12.2 \mathrm{~Hz}), 1.53-1.64(\mathrm{~m}, 3 \mathrm{H}), 1.43-1.52$ $(\mathrm{m}, 2 \mathrm{H}) ;{ }^{13} \mathrm{C} \mathrm{NMR}\left(100 \mathrm{MHz}, \mathrm{CDCl}_{3}\right) \delta 174.0,164.2,152.9,120.6,56.6,51.8,44.0$, 36.3, 34.7, 33.9, 33.6, 26.3, 24.6, 23.2; IR ( NaCl, $\left.\mathrm{CDCl}_{3}\right)$ 1734, 1661, 1615, 1439, 1354, 1326, 1195, 1161, $866 \mathrm{~cm}^{-1}$; HRMS $\left[\mathrm{C}_{14} \mathrm{H}_{22} \mathrm{NO}_{3}\right]^{+}$calcd 252.1600. Found 252.1588 $(\mathrm{FAB}+)$.

From the same reaction mixture, the minor product $\mathbf{4 q}(6.0 \mathrm{mg}, 9 \%)$ was also isolated but not fully characterized due to its small quantity.

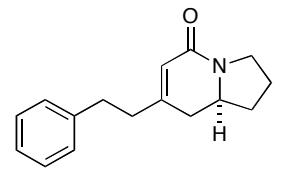

(S)-7-phenethyl-2,3,8,8a-tetrahydroindolizin-5(1H)-one

(3r). According to the general procedure with ligand L4, alkyne 1r, and isocyanate 2. Upon purification $32.6 \mathrm{mg}(50 \%)$ of the desired product was isolated: $\mathrm{R}_{\mathrm{f}}=0.38\left(5 \% \mathrm{MeOH} / \mathrm{CH}_{2} \mathrm{Cl}_{2}\right) ;[\alpha]_{\mathrm{D}}{ }^{20}=+101.9(\mathrm{c}=1.0$, $\mathrm{CHCl}_{3}$ ); HPLC analysis - Chiracel AD-H column 90:10 hexane:iPrOH, $1.0 \mathrm{ml} / \mathrm{min}$, Major: 15.0 minutes, Minor: 13.7 minutes, $210 \mathrm{~nm}$ detection light, $e e=84 \%$; ${ }^{1} \mathrm{H} \mathrm{NMR}$ $\left(400 \mathrm{MHz}, \mathrm{CDCl}_{3}\right) \delta 7.27(\mathrm{~d}, 1 \mathrm{H}, J=7.0 \mathrm{~Hz}), 7.25(\mathrm{~d}, 1 \mathrm{H}, J=7.7 \mathrm{~Hz}), 7.13-7.19(\mathrm{~m}$, $3 \mathrm{H}), 5.74(\mathrm{~m}, 1 \mathrm{H}), 3.63$ (dddd, $1 \mathrm{H}, J=5.3,5.3,10.4,15.4 \mathrm{~Hz}), 3.59$ (m, 1H), 3.42 (ddd, $1 \mathrm{H}, J=7.9,9.8,11.7 \mathrm{~Hz}), 2.77(\mathrm{dd}, 1 \mathrm{H}, J=7.2,7.2 \mathrm{~Hz}), 2.77(\mathrm{dd}, 1 \mathrm{H}, J=8.5,8.5 \mathrm{~Hz})$, $2.46(\mathrm{dd}, 1 \mathrm{H}, J=8.5,8.5 \mathrm{~Hz}), 2.46(\mathrm{dd}, 1 \mathrm{H}, J=7.4,7.4 \mathrm{~Hz}), 2.28(\mathrm{dd}, 1 \mathrm{H}, J=4.9,16.6$ $\mathrm{Hz}), 2.08-2.20(\mathrm{~m}, 2 \mathrm{H}), 1.99(\mathrm{~m}, 1 \mathrm{H}), 1.76$ (ddddd, $1 \mathrm{H}, J=6.6,9.6,9.6,12.2,12.2 \mathrm{~Hz}$ ), $1.57(\mathrm{~m}, 1 \mathrm{H}) ;{ }^{13} \mathrm{C}$ NMR $\left(100 \mathrm{MHz}, \mathrm{CDCl}_{3}\right) \delta 164.2,152.5,141.0,128.7,128.5,126.4$, 120.9, 56.6, 44.0, 38.4, 35.0, 33.6, 33.5, 23.2; IR ( NaCl, $\left.\mathrm{CDCl}_{3}\right)$ 1655, 1609, 1445, 1347, 1327, 1240, $856 \mathrm{~cm}^{-1}$; HRMS $\left[\mathrm{C}_{16} \mathrm{H}_{20} \mathrm{NO}\right]^{+}$calcd 245.1545. Found 245.1547 (FAB+).

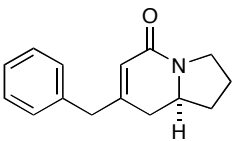

(S)-7-benzyl-2,3,8,8a-tetrahydroindolizin-5(1H)-one (3s). According to the general procedure with ligand $\mathbf{L 4}$, alkyne $\mathbf{1 s}$, and isocyanate $\mathbf{2}$. Upon purification $28.6 \mathrm{mg}(47 \%)$ of the desired product was isolated: $\mathrm{R}_{\mathrm{f}}$ $=0.13($ EtOAc $) ;[\alpha]_{\mathrm{D}}{ }^{20}=+81.5\left(\mathrm{c}=1.0, \mathrm{CHCl}_{3}\right)$; HPLC analysis Chiracel AD-H column 95:5 hexane:iPrOH, $1.0 \mathrm{ml} / \mathrm{min}$, Major: 30.3 minutes, Minor: 29.2 minutes, $210 \mathrm{~nm}$ detection light, ee $=84 \%$; ${ }^{1} \mathrm{H}$ NMR $\left(400 \mathrm{MHz}, \mathrm{CDCl}_{3}\right) \delta 7.12-$ $7.32(\mathrm{~m}, 5 \mathrm{H}), 5.75(\mathrm{~m}, 1 \mathrm{H}), 3.55-3.66(\mathrm{~m}, 2 \mathrm{H}), 3.37-3.50(\mathrm{~m}, 3 \mathrm{H}), 2.25(\mathrm{dd}, 1 \mathrm{H}, J=$ 4.9, $16.6 \mathrm{~Hz}$ ), $2.02-2.15(\mathrm{~m}, 2 \mathrm{H}), 1.97(\mathrm{~m}, 1 \mathrm{H}), 1.74$ (ddddd, $1 \mathrm{H}, J=6.8,9.6,9.6,12.2$, $12.2 \mathrm{~Hz}), 1.52(\mathrm{~m}, 1 \mathrm{H}) ;{ }^{13} \mathrm{C} \mathrm{NMR}\left(100 \mathrm{MHz}, \mathrm{CDCl}_{3}\right) \delta 164.1,152.0,137.4,129.3,128.9$, 127.0, 122.1, 56.8, 44.0, 43.2, 34.2, 33.6, 23.2; IR ( $\left.\mathrm{NaCl}, \mathrm{CH}_{2} \mathrm{Cl}_{2}\right)$ 1664, 1605, 1445, $1347,1320 \mathrm{~cm}^{-1}$; HRMS $\left[\mathrm{C}_{15} \mathrm{H}_{18} \mathrm{NO}\right]^{+}$calcd 228.1388. Found 228.1383 (FAB+).

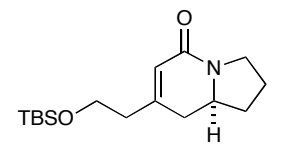

(S)-7-(2-(tert-butyldimethylsilyloxy)ethyl)-2,3,8,8atetrahydroindolizin-5(1H)-one (3t). According to the general procedure with ligand $\mathbf{L 4}$, alkyne $\mathbf{1 t}$, and isocyanate $\mathbf{2}$. Upon purification $51.9 \mathrm{mg}(65 \%)$ of the desired product was isolated: $\mathrm{R}_{\mathrm{f}}=0.13$ (EtOAc); $[\alpha]_{\mathrm{D}}{ }^{20}=+75.4\left(\mathrm{c}=1.0, \mathrm{CHCl}_{3}\right)$; HPLC analysis - Chiracel AD-H column 99:1 hexane:iPrOH, $1.0 \mathrm{ml} / \mathrm{min}$, Major: 18.2 minutes, Minor: 16.1 minutes, $210 \mathrm{~nm}$ detection light, ee $=87 \% ;{ }^{1} \mathrm{H}$ NMR $\left(400 \mathrm{MHz}, \mathrm{CDCl}_{3}\right) \delta 5.71(\mathrm{~m}, 1 \mathrm{H}), 3.72(\mathrm{t}, 2 \mathrm{H}, J=6.6 \mathrm{~Hz})$, 3.64 (dddd, $1 \mathrm{H}, J=5.3,5.3,10.4,15.1 \mathrm{~Hz}$ ), 3.59 (ddd, $1 \mathrm{H}, J=2.1,9.2,11.5 \mathrm{~Hz}$ ), 3.42 (ddd, 1H, $J=7.7,9.8,11.7 \mathrm{~Hz}), 2.38(\mathrm{dd}, 1 \mathrm{H}, J=4.7,16.6 \mathrm{~Hz}), 2.32-2.36(\mathrm{~m}, 2 \mathrm{H})$, 
$2.08-2.19(\mathrm{~m}, 2 \mathrm{H}), 1.98(\mathrm{~m}, 1 \mathrm{H}), 1.76$ (ddddd, $1 \mathrm{H}, J=6.6,9.4,9.4,11.9,11.9 \mathrm{~Hz}), 1.56$ $(\mathrm{m}, 1 \mathrm{H}), 0.84(\mathrm{~s}, 9 \mathrm{H}), 0.01(\mathrm{~s}, 6 \mathrm{H}) ;{ }^{13} \mathrm{C} \mathrm{NMR}\left(100 \mathrm{MHz}, \mathrm{CDCl}_{3}\right) \delta$ 164.1, 151.0, 121.9, 61.6, 56.6, 44.0, 39.8, 35.2, 33.6, 26.1, 23.2, 18.4, -5.2; IR $\left(\mathrm{NaCl}, \mathrm{CDCl}_{3}\right)$ 1660, 1598, 1455, 1352, 1327, 1245, 1091, 1050, $835 \mathrm{~cm}^{-1}$; HRMS $\left[\mathrm{C}_{16} \mathrm{H}_{30} \mathrm{NO}_{2} \mathrm{Si}\right]^{+}$calcd 296.2046. Found 296.2057 (FAB+).

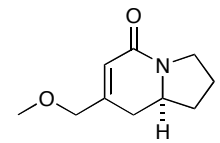

(S)-7-(methoxymethyl)-2,3,8,8a-tetrahydroindolizin-5(1H)-one (3u). According to the general procedure with ligand L4, alkyne 1u, and isocyanate 2 (seal tube). Upon purification $22.6 \mathrm{mg}(46 \%)$ of the desired product was isolated: $\mathrm{R}_{\mathrm{f}}=0.07(\mathrm{EtOAc}) ;[\alpha]_{\mathrm{D}}{ }^{20}=+66.9\left(\mathrm{c}=1.0, \mathrm{CHCl}_{3}\right) ; \mathrm{HPLC}$ analysis - Chiracel OD-H column 80:20 hexane:iPrOH, $1.0 \mathrm{ml} / \mathrm{min}$, Major: 9.1 minutes, Minor: 10.2 minutes, $210 \mathrm{~nm}$ detection light, ee $=77 \%$; ${ }^{1} \mathrm{H}$ NMR $\left(400 \mathrm{MHz}, \mathrm{CDCl}_{3}\right) \delta 5.91(\mathrm{~m}$, $1 \mathrm{H}), 3.98(\mathrm{~d}, 1 \mathrm{H}, J=13.9 \mathrm{~Hz}), 3.92(\mathrm{~d}, 1 \mathrm{H}, J=13.9 \mathrm{~Hz}), 3.67$ (dddd, 1H, $J=5.1,5.1$, 10.4, 15.1 Hz), $3.61(\mathrm{ddd}, 1 \mathrm{H}, J=2.1,9.2,11.5 \mathrm{~Hz}), 3.44(\mathrm{~m}, 1 \mathrm{H}), 3.31(\mathrm{~s}, 3 \mathrm{H}), 2.42$ (dd, $1 \mathrm{H}, J=4.7,16.6 \mathrm{~Hz}), 2.19(\mathrm{ddd}, 1 \mathrm{H}, J=6.2,6.2,11.7 \mathrm{~Hz}), 1.96-2.11(\mathrm{~m}, 2 \mathrm{H}), 1.77$ (ddddd, $1 \mathrm{H}, J=6.8,9.8,9.8,12.4,12.4 \mathrm{~Hz}), 1.60(\mathrm{~m}, 1 \mathrm{H}) ;{ }^{13} \mathrm{C} \mathrm{NMR}\left(100 \mathrm{MHz}, \mathrm{CDCl}_{3}\right)$ $\delta$ 163.7, 148.9, 121.4, 74.0, 58.6, 56.7, 44.1, 33.6, 31.5, 23.2; IR ( $\left.\mathrm{NaCl}, \mathrm{CDCl}_{3}\right)$ 1667, $1610,1449,1340,1191,1105 \mathrm{~cm}^{-1}$; HRMS $\left[\mathrm{C}_{10} \mathrm{H}_{16} \mathrm{NO}_{2}\right]^{+}$calcd 182.1181. Found 182.1181 (FAB+).

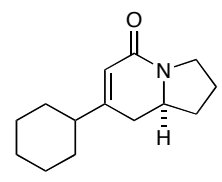

(S)-7-cyclohexyl-2,3,8,8a-tetrahydroindolizin-5(1H)-one

(3v). According to the general procedure with ligand $\mathbf{L 3}$, alkyne $\mathbf{1 v}$, and isocyanate 2. Upon purification $26.2 \mathrm{mg}(44 \%)$ of the desired product was isolated: $\mathrm{R}_{\mathrm{f}}=0.17\left(\right.$ EtOAc); $[\alpha]_{\mathrm{D}}{ }^{20}=+68.9\left(\mathrm{c}=1.0, \mathrm{CHCl}_{3}\right) ; \mathrm{HPLC}$ analysis - Chiracel AD-H column 95:5 hexane:iPrOH, $1.0 \mathrm{ml} / \mathrm{min}$, Major: 39.3 minutes, Minor: 29.3 minutes, $210 \mathrm{~nm}$ detection light, ee $=76 \% ;{ }^{1} \mathrm{H} \mathrm{NMR}\left(400 \mathrm{MHz}, \mathrm{CDCl}_{3}\right) \delta$ $5.67(\mathrm{~m}, 1 \mathrm{H}), 3.54-3.64(\mathrm{~m}, 2 \mathrm{H}), 3.42(\mathrm{ddd}, 1 \mathrm{H}, J=7.7,9.6,11.7 \mathrm{~Hz}), 2.32(\mathrm{dd}, 1 \mathrm{H}, J=$ 4.7, $16.4 \mathrm{~Hz}), 2.16(\mathrm{ddd}, 1 \mathrm{H}, J=5.5,5.5,11.9 \mathrm{~Hz}), 2.09$ (ddd, $1 \mathrm{H}, J=2.6,14.5,16.6$ $\mathrm{Hz}), 1.93-2.02(\mathrm{~m}, 2 \mathrm{H}), 1.63-1.81(\mathrm{~m}, 6 \mathrm{H}), 1.57(\mathrm{~m}, 1 \mathrm{H}), 1.07-1.31(\mathrm{~m}, 5 \mathrm{H}) ;{ }^{13} \mathrm{C}$ NMR $\left(100 \mathrm{MHz}, \mathrm{CDCl}_{3}\right) \delta 164.7,158.2,118.6,56.8,44.8,44.0,33.7,33.5,31.3,30.9$, 26.5, 26.4, 26.3, 23.2; IR $\left(\mathrm{NaCl}, \mathrm{CH}_{2} \mathrm{Cl}_{2}\right)$ 1655, 1603, 1445, 1332, 1163, $851 \mathrm{~cm}^{-1}$; HRMS $\left[\mathrm{C}_{14} \mathrm{H}_{22} \mathrm{NO}\right]^{+}$calcd 220.1701. Found $220.1712(\mathrm{FAB}+)$.

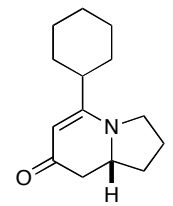

(R)-5-cyclohexyl-2,3,8,8a-tetrahydroindolizin-7(1H)-one $(4 \mathrm{v})$. From the same reaction as above, upon purification $22.4 \mathrm{mg}(38 \%)$ of the desired product was isolated: $\mathrm{R}_{\mathrm{f}}=0.07(\mathrm{EtOAc}) ;[\alpha]_{\mathrm{D}}{ }^{20}=+432.3\left(\mathrm{c}=1.0, \mathrm{CHCl}_{3}\right)$; HPLC analysis - Chiracel AD-H column 90:10 hexane:iPrOH, $1.0 \mathrm{ml} / \mathrm{min}$, Major: 16.5 minutes, Minor: 15.7 minutes, $330 \mathrm{~nm}$ detection light, ee $=95 \%$; ${ }^{1} \mathrm{H}$ NMR (400 MHz, $\mathrm{CDCl}_{3}$ ) $\delta 4.96(\mathrm{~s}, 1 \mathrm{H}), 3.68$ (dddd, $1 \mathrm{H}, J=5.3,5.3,10.4,15.8 \mathrm{~Hz}$ ), $3.59(\mathrm{~m}, 1 \mathrm{H}), 3.42(\mathrm{~m}, 1 \mathrm{H}), 2.35(\mathrm{dd}, 1 \mathrm{H}, J=4.7,15.8 \mathrm{~Hz}), 2.13-2.27(\mathrm{~m}, 3 \mathrm{H}), 2.07$ (m, $1 \mathrm{H}), 1.58-1.91(\mathrm{~m}, 7 \mathrm{H}), 1.14-1.32(\mathrm{~m}, 5 \mathrm{H}) ;{ }^{13} \mathrm{C} \mathrm{NMR}\left(100 \mathrm{MHz}, \mathrm{CDCl}_{3}\right) \delta 192.3$, 169.0, 94.9, 59.4, 46.5, 41.9, 41.5, 32.6, 31.4, 31.3, 26.6, 26.4, 26.0, 24.0; IR ( NaCl, $\left.\mathrm{CDCl}_{3}\right) 1624,1537,1481,1342,1260,1245,1128 \mathrm{~cm}^{-1} ;$ HRMS $\left[\mathrm{C}_{14} \mathrm{H}_{22} \mathrm{NO}\right]^{+}$calcd 220.1701. Found 220.1699 (FAB+). 


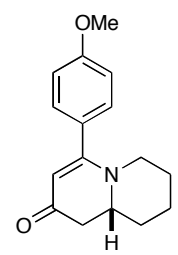

(R)-4-(4-methoxyphenyl)-7,8,9,9a-tetrahydro-1H-quinolizin-2(6H)-one (6c). According to the general procedure with ligand L5, alkyne 1c, and isocyanate 5. Upon purification $31.3 \mathrm{mg}(45 \%)$ of the desired product was isolated: $\mathrm{R}_{\mathrm{f}}=0.22($ EtOAc $) ;[\alpha]_{\mathrm{D}}^{20}=+52\left(\mathrm{c}=1.0, \mathrm{CHCl}_{3}\right)$; HPLC analysis Chiracel AD-H column 90:10 hexane:iPrOH, $1.0 \mathrm{ml} / \mathrm{min}$, Major: 13.3 minutes, Minor: 15.8 minutes, $330 \mathrm{~nm}$ detection light, ee $=98 \%$; ${ }^{1} \mathrm{H}$ NMR $\left(400 \mathrm{MHz}, \mathrm{CDCl}_{3}\right) \delta 7.19$ (br d, $2 \mathrm{H}, J=8.7 \mathrm{~Hz}$ ), 6.89 (br d, 2H, $\left.J=8.5 \mathrm{~Hz}\right), 5.02(\mathrm{~s}, 1 \mathrm{H})$, $3.80(\mathrm{~s}, 3 \mathrm{H}), 3.60(\mathrm{~m}, 1 \mathrm{H}), 3.44(\mathrm{dddd}, 1 \mathrm{H}, J=6.0,6.0,11.3,11.3 \mathrm{~Hz}), 2.57(\mathrm{dd}, 1 \mathrm{H}, J=$ 5.8, 16.4 Hz), $2.56(\mathrm{~m}, 1 \mathrm{H}), 2.40(\mathrm{dd}, 1 \mathrm{H}, J=11.3,16.4 \mathrm{~Hz}), 1.83(\mathrm{~m}, 1 \mathrm{H}), 1.69-1.75$ $(\mathrm{m}, 2 \mathrm{H}), 1.38$ - $1.56(\mathrm{~m}, 3 \mathrm{H}) ;{ }^{13} \mathrm{C}$ NMR $\left(100 \mathrm{MHz}, \mathrm{CDCl}_{3}\right) \delta$ 191.8, 166.1, 160.3, 129.2, $128.7,114.2$, 103.6, 58.8, 55.5, 50.6, 42.8, 31.6, 26.2, 24.1; IR $\left(\mathrm{NaCl}, \mathrm{CDCl}_{3}\right) 1639$, 1603, 1547, 1511, 1434, 1245, 1178, $1132 \mathrm{~cm}^{-1}$; HRMS $\left[\mathrm{C}_{16} \mathrm{H}_{20} \mathrm{NO}_{2}\right]^{+}$calcd 258.1494. Found 258.1484 (FAB+).

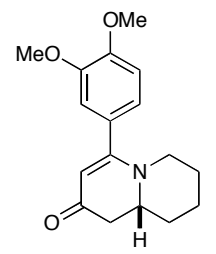

(R)-4-(3,4-dimethoxyphenyl)-7,8,9,9a-tetrahydro-1 $H$-quinolizin-2(6H)one (6b). According to the general procedure with ligand L5, alkyne 1b, and isocyanate 5. Upon purification $47.9 \mathrm{mg}(62 \%)$ of the desired product was isolated: $\mathrm{R}_{\mathrm{f}}=0.15(\mathrm{EtOAc}) ;[\alpha]_{\mathrm{D}}{ }^{20}=+54.5\left(\mathrm{c}=1.0, \mathrm{CHCl}_{3}\right) ; \mathrm{HPLC}$ analysis - Chiracel AD-H column 80:20 hexane:iPrOH, $1.0 \mathrm{ml} / \mathrm{min}$, Major: 20.6 minutes, Minor: 22.4 minutes, $330 \mathrm{~nm}$ detection light, $e e=98 \% ;{ }^{1} \mathrm{H}$ NMR $\left(400 \mathrm{MHz}, \mathrm{CDCl}_{3}\right) \delta 6.85(\mathrm{~d}, 1 \mathrm{H}, J=8.1 \mathrm{~Hz}), 6.82(\mathrm{dd}, 1 \mathrm{H}, J=1.7,8.1 \mathrm{~Hz}), 6.76$ $(\mathrm{d}, 1 \mathrm{H}, J=1.7 \mathrm{~Hz}), 5.07(\mathrm{~s}, 1 \mathrm{H}), 3.87(\mathrm{~s}, 3 \mathrm{H}), 3.85(\mathrm{~s}, 3 \mathrm{H}), 3.63(\mathrm{~m}, 1 \mathrm{H}), 3.44$ (dddd, $1 \mathrm{H}$, $J=6.0,6.0,11.3,11.3 \mathrm{~Hz}), 2.58(\mathrm{~m}, 1 \mathrm{H}), 2.57(\mathrm{dd}, 1 \mathrm{H}, J=5.8,16.2 \mathrm{~Hz}), 2.40(\mathrm{dd}, 1 \mathrm{H}, J$ $=11.1,16.2 \mathrm{~Hz}), 1.84(\mathrm{~m}, 1 \mathrm{H}), 1.69-1.76(\mathrm{~m}, 2 \mathrm{H}), 1.38-1.58(\mathrm{~m}, 3 \mathrm{H}) ;{ }^{13} \mathrm{C} \mathrm{NMR}(100$ $\left.\mathrm{MHz}, \mathrm{CDCl}_{3}\right) \delta 191.8,166.1,149.7,149.1,129.4,119.9,111.2,110.4,103.5,58.9,56.2$, 56.1, 50.7, 42.7, 31.6, 26.2, 24.1; IR ( $\left.\mathrm{NaCl}, \mathrm{CDCl}_{3}\right)$ 1634, 1547, 1511, 1440, 1342, 1250, $1137,1025 \mathrm{~cm}^{-1}$; HRMS $\left[\mathrm{C}_{17} \mathrm{H}_{22} \mathrm{NO}_{3}\right]^{+}$calcd 288.1600. Found 288.1591 (FAB+).

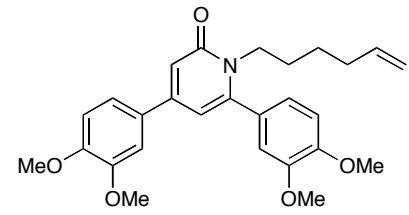

4,6-bis(3,4-dimethoxyphenyl)-1-(hex-5-enyl)pyridin-2(1H)one $(\mathbf{7 b})$. From the same reaction as above, the pyridone side product (ca. $27 \mathrm{mg}, 22 \%$ ) was isolated and characterized: $\mathrm{R}_{\mathrm{f}}=$ 0.41 (EtOAc); ${ }^{1} \mathrm{H}$ NMR (400 MHz, $\mathrm{CDCl}_{3}$ ) $\delta 7.16$ (dd, $1 \mathrm{H}, J=$ $2.1,8.5 \mathrm{~Hz}), 7.08(\mathrm{~d}, 1 \mathrm{H}, J=2.1 \mathrm{~Hz}), 6.92-6.94(\mathrm{~m}, 2 \mathrm{H}), 6.88$ $(\mathrm{d}, 1 \mathrm{H}, J=8.5 \mathrm{~Hz}), 6.85(\mathrm{~s}, 1 \mathrm{H}), 6.74(\mathrm{~d}, 1 \mathrm{H}, J=2.1 \mathrm{~Hz}), 6.30(\mathrm{~d}, 1 \mathrm{H}, J=2.1 \mathrm{~Hz}), 5.66$ (dddd, 1H, $J=6.6,6.6,10.5,16.8 \mathrm{~Hz}), 4.84-4.92(\mathrm{~m}, 2 \mathrm{H}), 3.93(\mathrm{~s}, 3 \mathrm{H}), 3.89(\mathrm{~s}, 3 \mathrm{H})$, $3.89(\mathrm{~s}, 3 \mathrm{H}), 3.88(\mathrm{~s}, 3 \mathrm{H}), 1.90(\mathrm{q}, 2 \mathrm{H}, J=7.0 \mathrm{~Hz}), 1.60(\mathrm{~m}, 2 \mathrm{H}), 1.23(\mathrm{~m}, 2 \mathrm{H}) ;{ }^{13} \mathrm{C} \mathrm{NMR}$ $\left(100 \mathrm{MHz} \mathrm{CDCl}_{3}\right) \delta 163.7,150.4,150.0,149.8,149.5,149.4,148.9,138.5,130.3,128.5$, 121.5, 119.7, 114.9, 114.8, 111.9, 111.4, 111.0, 109.8, 107.6, 56.3, 56.2, 45.8, 33.4, 28.7, 26.2; IR $\left(\mathrm{NaCl}, \mathrm{CDCl}_{3}\right)$ 1650, 1603, 1583, 1506, 1455, 1265, 1137, $1025 \mathrm{~cm}^{-1}$; HRMS $\left[\mathrm{C}_{27} \mathrm{H}_{32} \mathrm{NO}_{5}\right]^{+}$calcd 450.2280. Found 450.2276(FAB+).

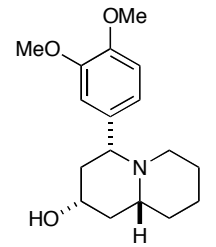

Hydrogenation of $6 \mathbf{b}(\mathbf{8})$. A mixture of $6 \mathbf{b}(66 \mathrm{mg}, 0.23 \mathrm{mmol})$ and $34 \mathrm{mg}$ of $10 \% \mathrm{Pd} / \mathrm{C}$ in $9 \mathrm{ml}$ of $\mathrm{MeOH}$ was stirred at ambient temperature under hydrogen atmosphere (1 atm) for 30 hours. The reaction mixture was filtered through celite and concentrated in vacuo. Upon purification by column chromatography $53.5 \mathrm{mg}(80 \%)$ of the desired product $\mathbf{8}$ was 
isolated: $\mathrm{R}_{\mathrm{f}}=0.07(\mathrm{EtOAc}) ;[\alpha]_{\mathrm{D}}{ }^{20}=+53.8\left(\mathrm{c}=1.0, \mathrm{CHCl}_{3}\right) ;{ }^{1} \mathrm{H}$ NMR $(300 \mathrm{MHz}$, $\left.\mathrm{CDCl}_{3}\right) \delta 6.60-7.10(\mathrm{~m}, 3 \mathrm{H}), 3.91(\mathrm{~s}, 3 \mathrm{H}), 3.87(\mathrm{~s}, 3 \mathrm{H}), 3.75(\mathrm{~m}, 1 \mathrm{H}), 2.93(\mathrm{br} \mathrm{d}, 1 \mathrm{H}, J=$ $10.8 \mathrm{~Hz}), 2.70(\mathrm{br} \mathrm{d}, 1 \mathrm{H}, J=10.9 \mathrm{~Hz}), 1.88-2.08(\mathrm{~m}, 3 \mathrm{H}), 1.33-1.80(\mathrm{~m}, 9 \mathrm{H}), 1.26(\mathrm{~m}$, $1 \mathrm{H}) ;{ }^{13} \mathrm{C}$ NMR $\left(75 \mathrm{MHz}, \mathrm{CDCl}_{3}\right) \delta 148.2,122.4,110.8,68.6,68.5,56.2,56.1,53.2,45.4$, 43.0, 33.8, 26.3, 24.8; IR ( $\left.\mathrm{NaCl}, \mathrm{CDCl}_{3}\right)$ 2934, 1592, 1506, 1455, 1363, 1260, 1225, 1128, 1025, 910, $727 \mathrm{~cm}^{-1}$; HRMS $\left[\mathrm{C}_{17} \mathrm{H}_{26} \mathrm{NO}_{3}\right]^{+}$calcd 292.1913. Found 292.1914 $(\mathrm{FAB}+)$. Spectral properties were in agreement with literature values reported for the opposite enantiomer. ${ }^{4}$

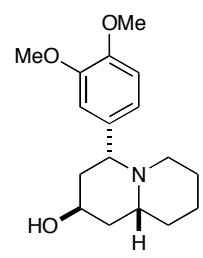

$(+)$-Lasubine II. According to the literature procedure, ${ }^{4}$ a Mitsunobu reaction on $8(42.0 \mathrm{mg}, 0.144 \mathrm{mmol})$ provided $27.0 \mathrm{mg}(64 \%)$ of the desired product: $\mathrm{R}_{\mathrm{f}}=0.24(8: 1 \mathrm{EtOAc} / \mathrm{MeOH}) ;[\alpha]_{\mathrm{D}}{ }^{20}=+43.4(\mathrm{c}=1.0$, $\left.\mathrm{CHCl}_{3}\right) ;{ }^{1} \mathrm{H} \mathrm{NMR}\left(400 \mathrm{MHz}, \mathrm{CDCl}_{3}\right) \delta 6.74-6.91(\mathrm{~m}, 3 \mathrm{H}), 4.12$ (br s, $\left.1 \mathrm{H}\right)$, $3.86(\mathrm{~s}, 3 \mathrm{H}), 3.83(\mathrm{~s}, 3 \mathrm{H}), 3.29(\mathrm{br} \mathrm{d}, 1 \mathrm{H}, J=10.2 \mathrm{~Hz}), 2.66(\mathrm{br} \mathrm{d}, 1 \mathrm{H}, J=$ $10.6 \mathrm{~Hz}), 2.36(\mathrm{~m}, 1 \mathrm{H}), 1.20-1.92(\mathrm{~m}, 12 \mathrm{H}) ;{ }^{13} \mathrm{C} \mathrm{NMR}\left(100 \mathrm{MHz}, \mathrm{CDCl}_{3}\right)$ $\delta$ 149.1, 148.0, 137.4, 119.9, 111.0, 107.0, 65.3, 63.6, 56.7, 56.2, 56.0, 53.4, 43.0, 40.5, 33.8, 26.3, 25.1; IR $\left(\mathrm{NaCl}, \mathrm{CDCl}_{3}\right) 2930,1588,1516,1460,1414,1260,1132,1025 \mathrm{~cm}^{-1}$; HRMS $\left[\mathrm{C}_{17} \mathrm{H}_{26} \mathrm{NO}_{3}\right]^{+}$calcd 292.1913. Found 292.1916 (FAB+). Spectral properties were in agreement with literature values reported for the (-)-lasubine II. $^{5}$

${ }^{1} \mathrm{H}$ NMR and ${ }^{13} \mathrm{C}$ NMR spectra of new compounds are attached:

$\mathrm{X}$-ray data of $\mathbf{3} \mathbf{j}$ and $\mathbf{4 j}$ are attached following the spectra

\footnotetext{
${ }^{4}$ Ma, D.; Zhu, W. Org. Lett. 2001, 3, 3927.

5 Back, T. G.; Hamilton, M. D. Org. Lett. 2002, 4, 1779.
} 

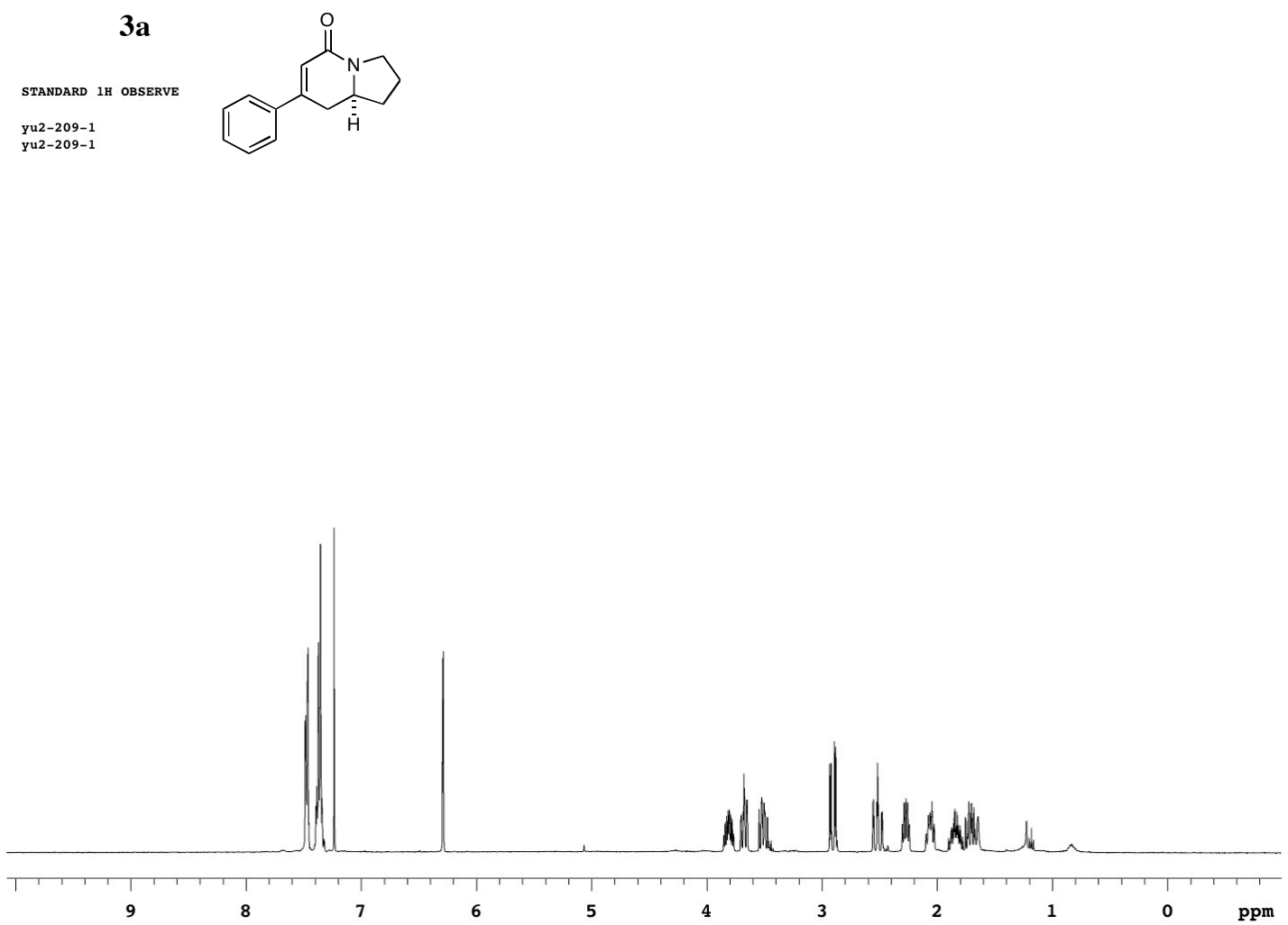

$13 \mathrm{C}$ OBSERVE

yu2-209-1

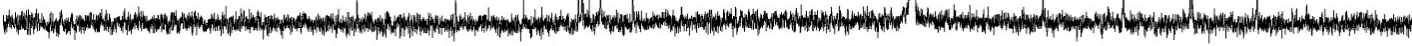




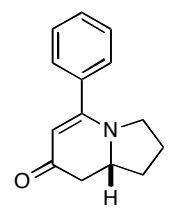

$4 a$

STANDARD $1 \mathrm{H}$ OBSERVE

yu2-209-2

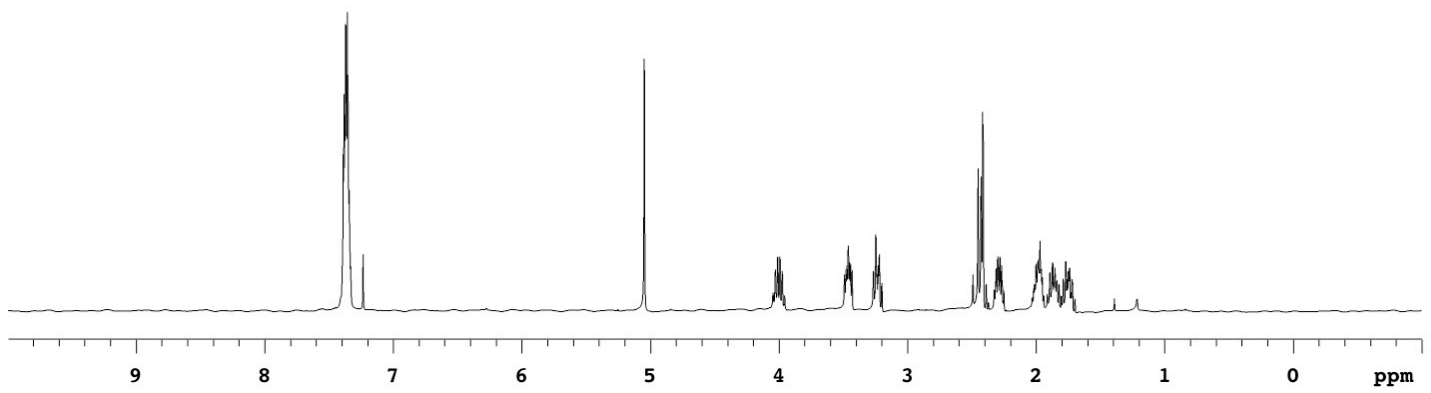

13C OBSERVE

yu2-209-2

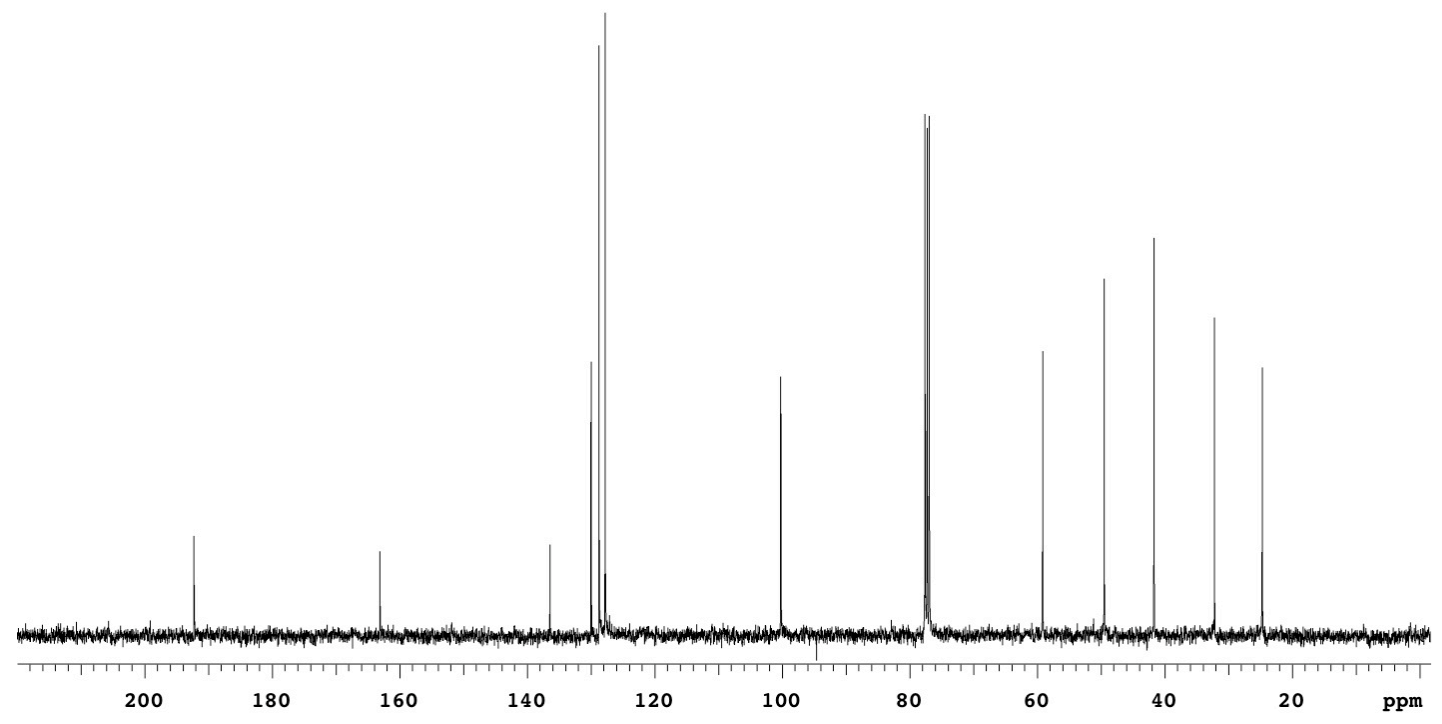

S-16 


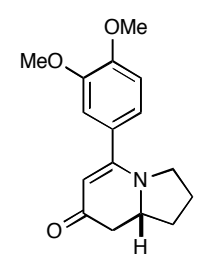

$4 b$

STANDARD 1H ObSERve

yu2-280

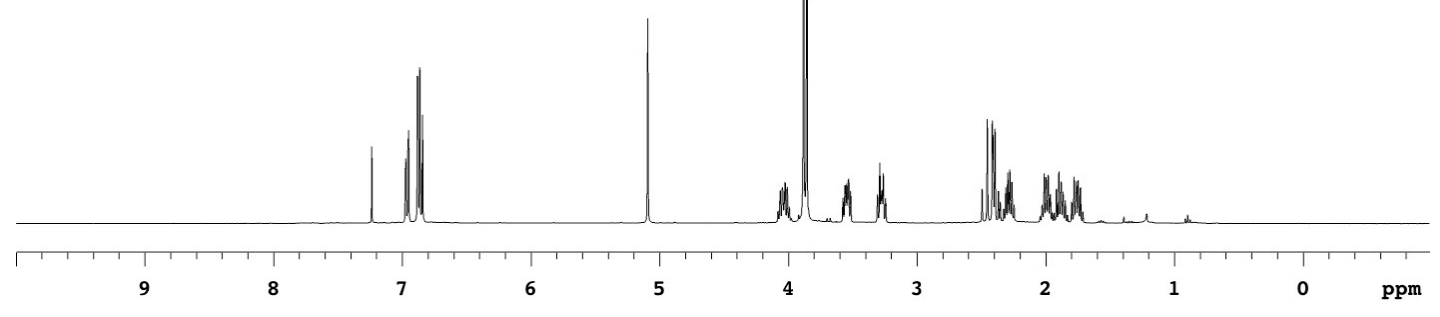

13C OBSERVE

yu2-280

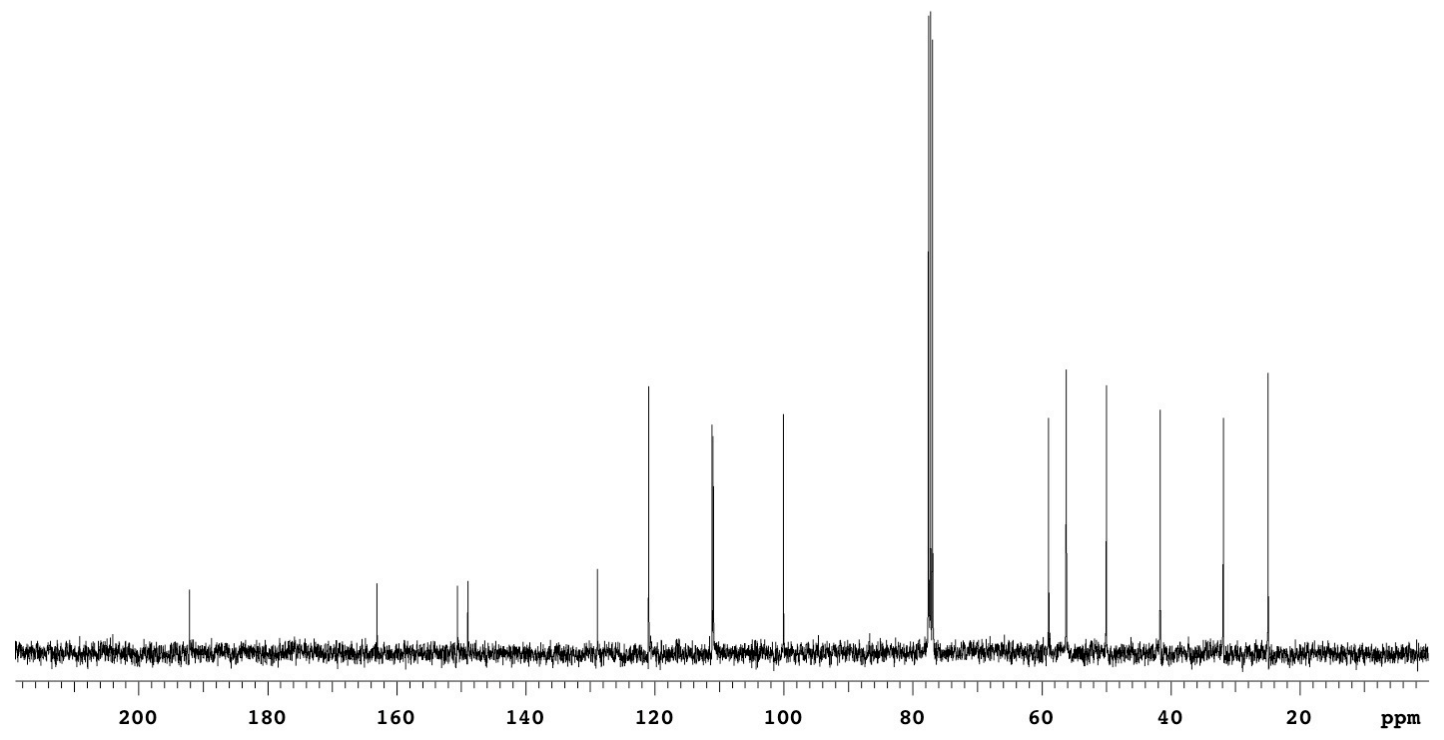

S-17 


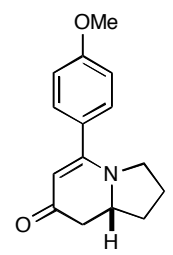

4c

STANDARD 1H OBSERVE

yu2-252
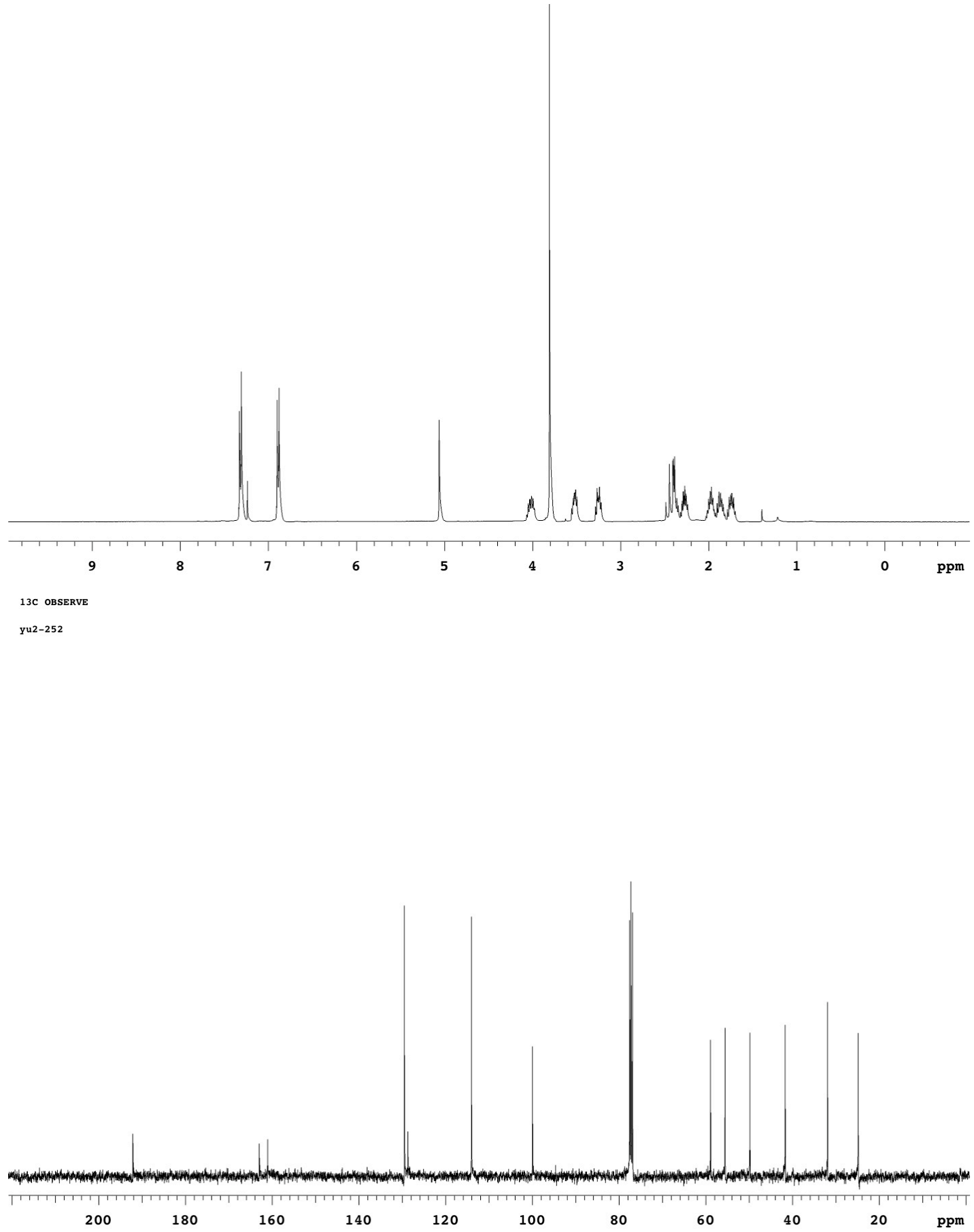

S-18 


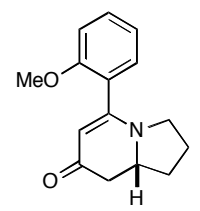

4d

STANDARD 1H OBSERVE

yu2-281

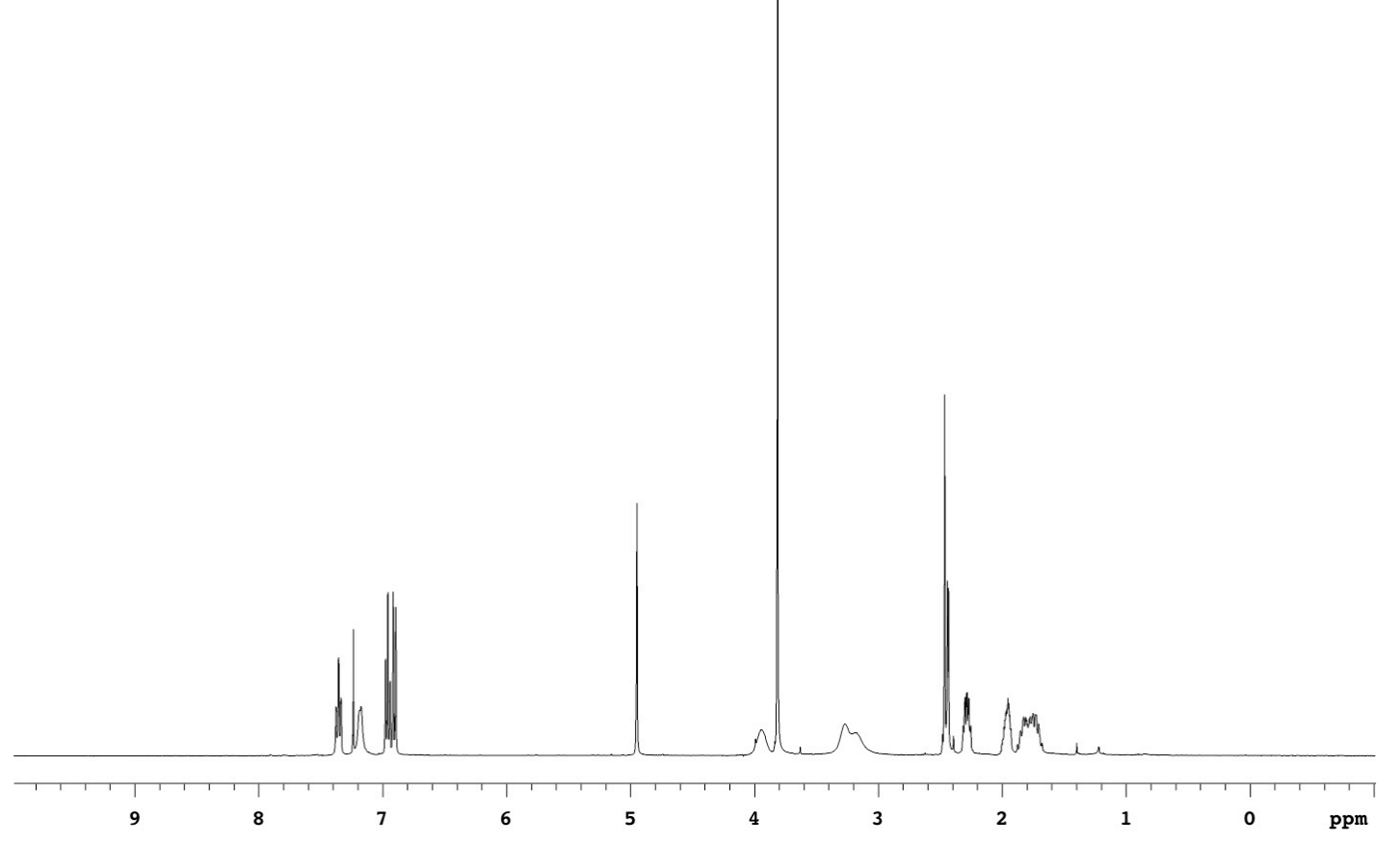

13C OBSERVE

yu2-281

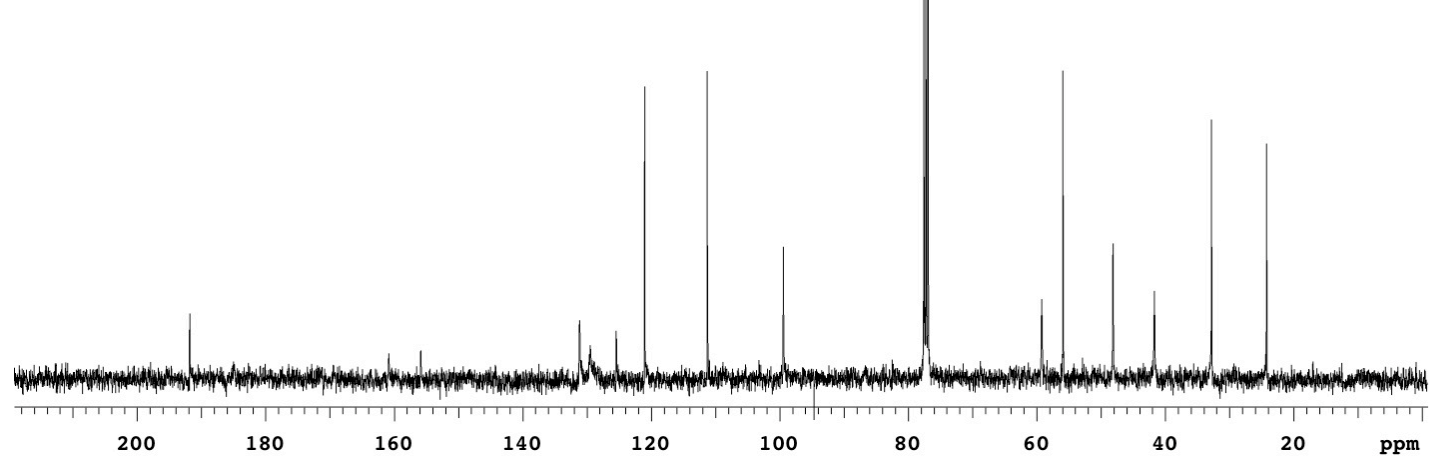

S-19 


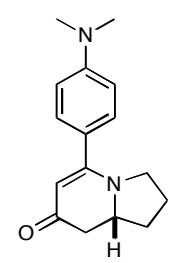

$4 e$

STANDARD $1 \mathrm{H}$ OBSERVE

Yu2-273

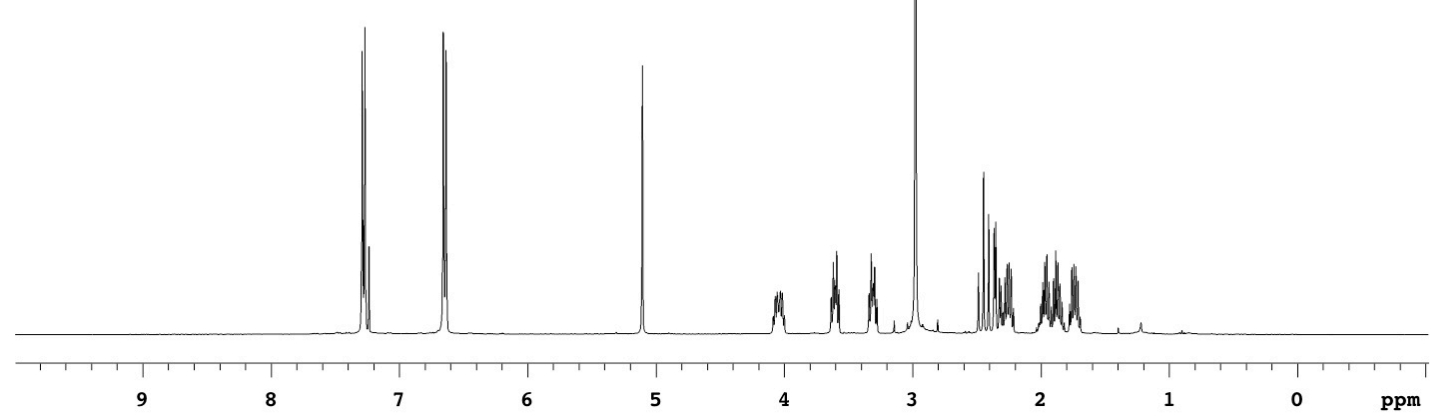

13C OBSERVE

yu2-273

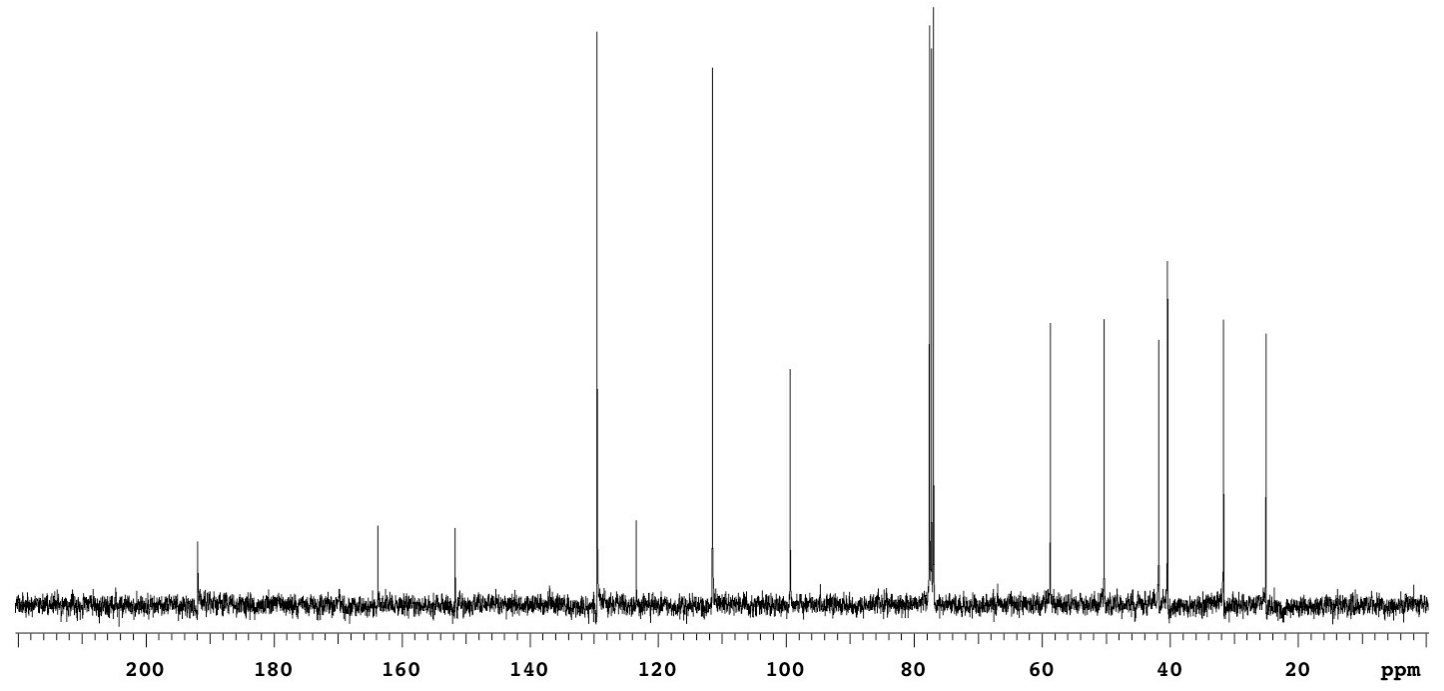

S-20 


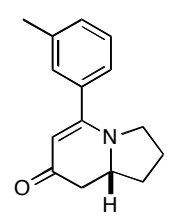

$4 f$

STANDARD $1 \mathrm{H}$ OBSERVE

yu2-258-2
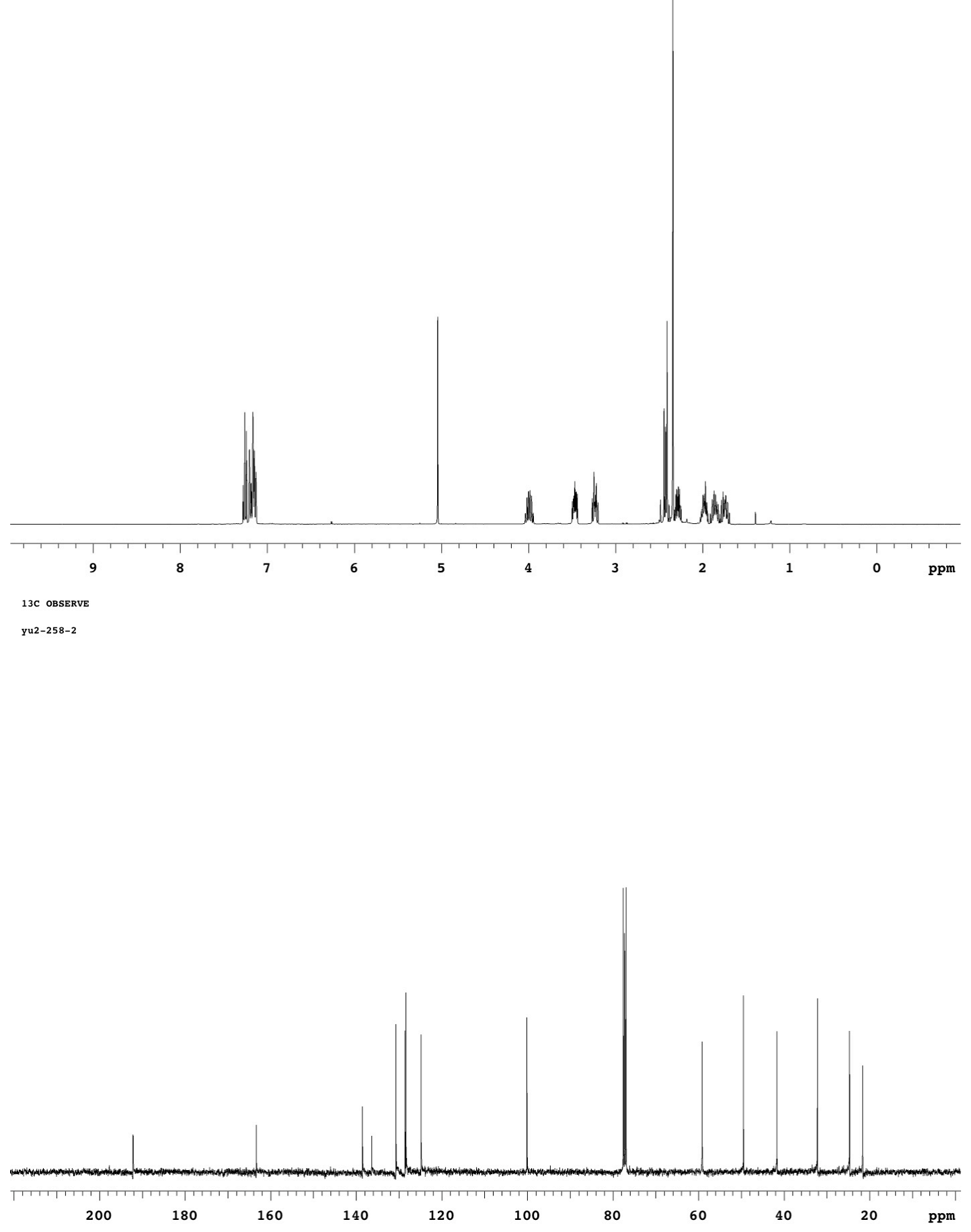

S-21 


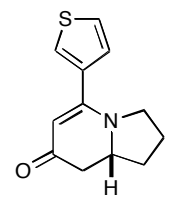

$4 \mathrm{~g}$

1H OBSERVE

yu2-260-2

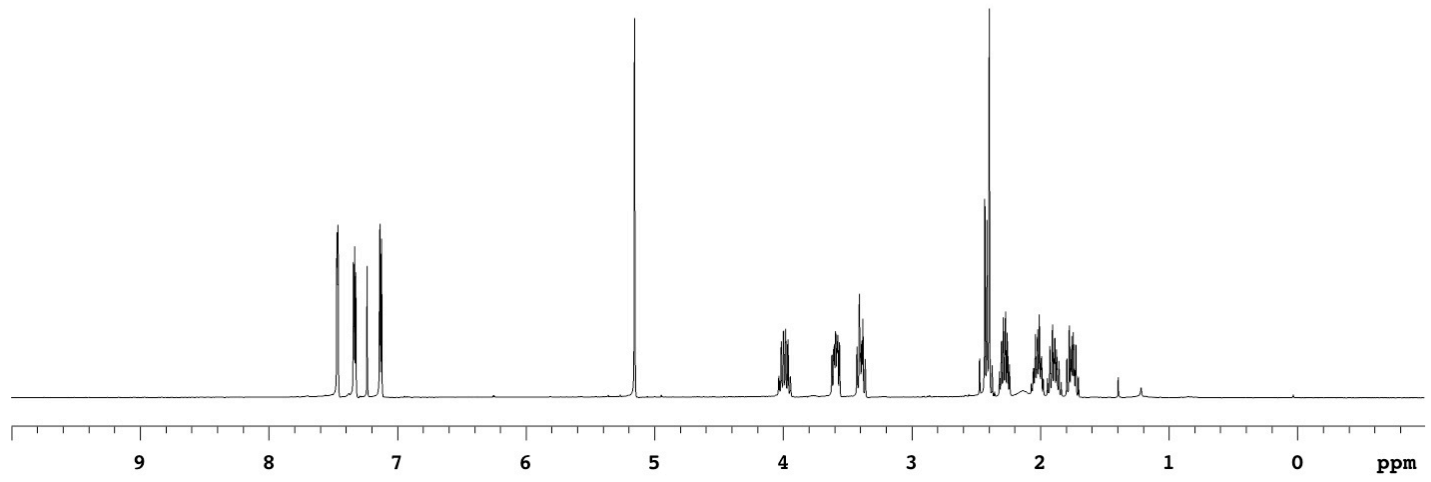

$13 \mathrm{C}$ OBSERVE

yu2-260-2

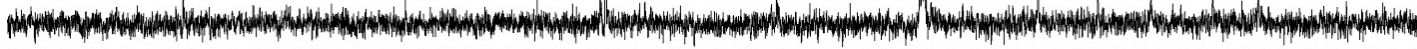

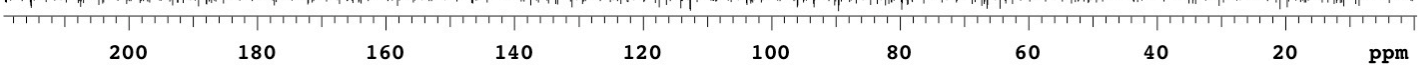




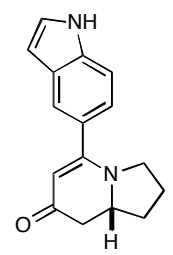

$4 h$

STANDARD $1 \mathrm{H}$ OBSERVE

yu2-288

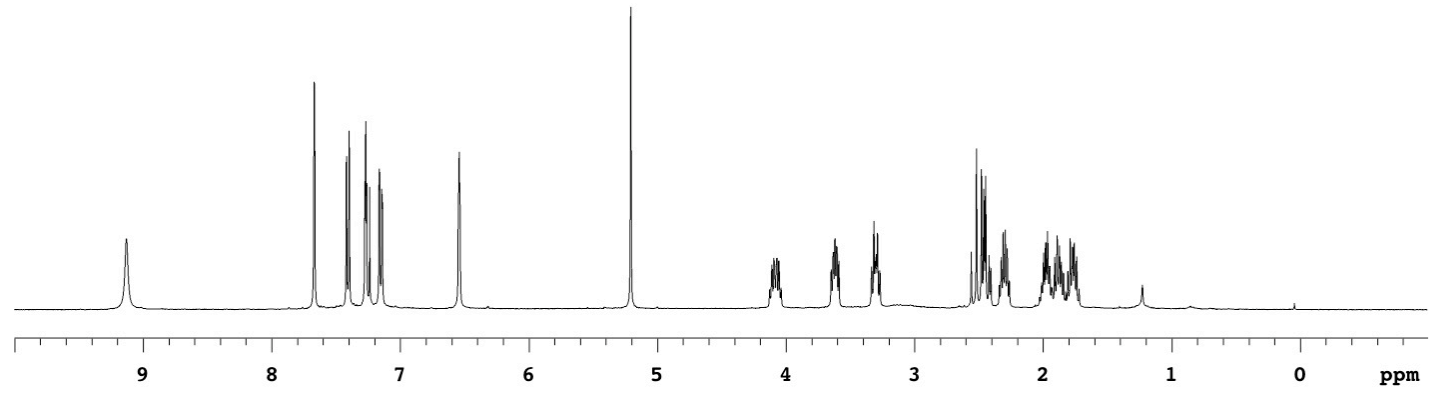

13C OBSERVE

yu2-288

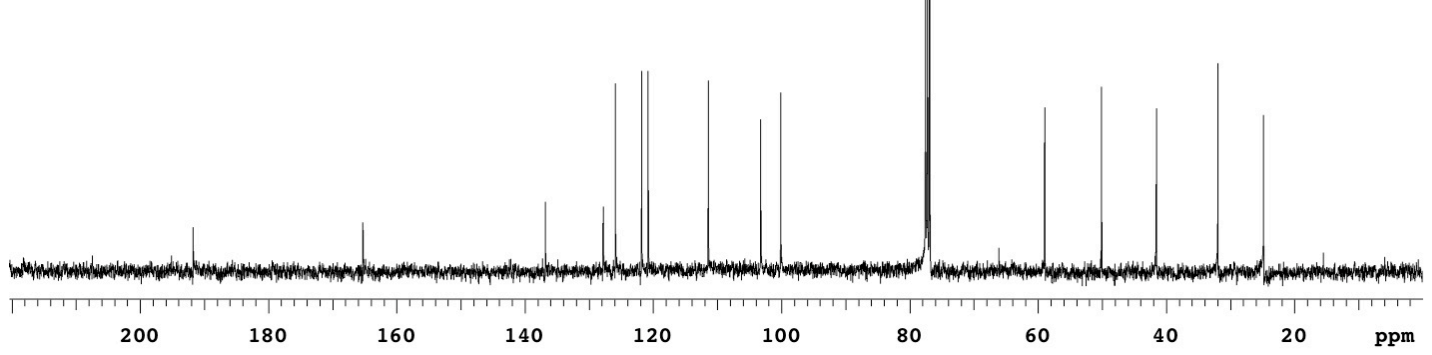

S-23 
$4 i$

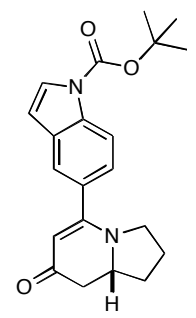

yu2-291
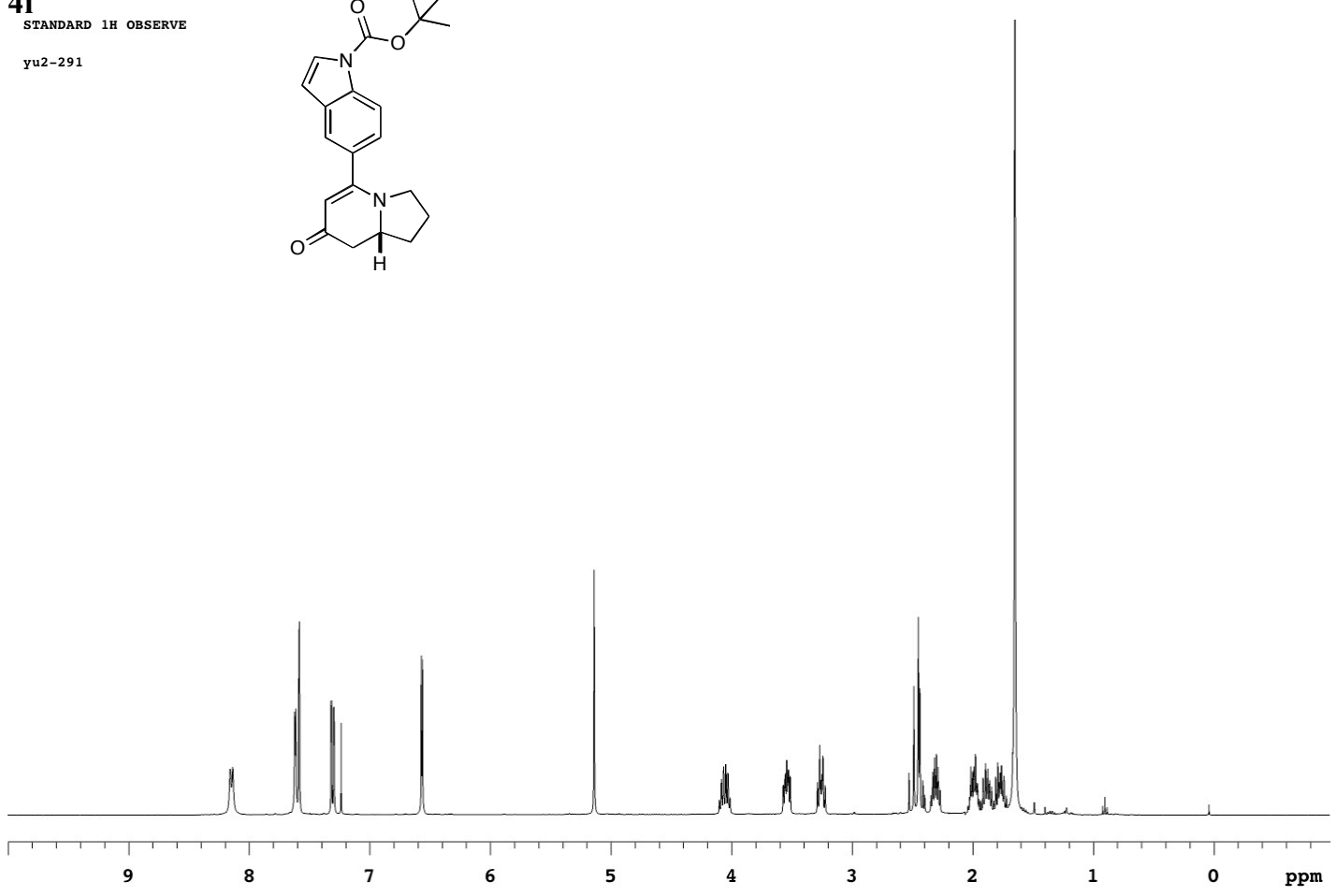

13C OBSERVE

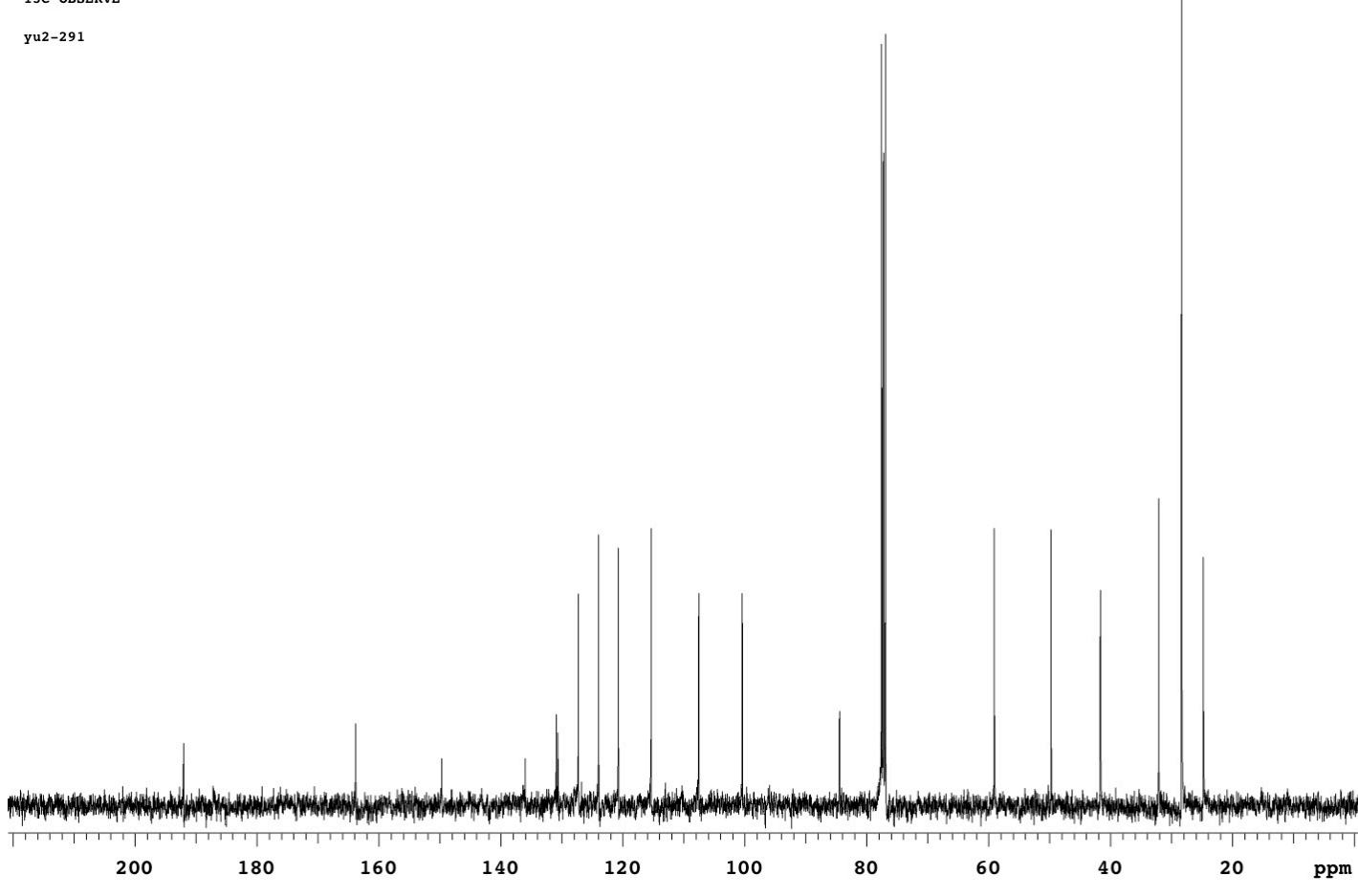

S-24 


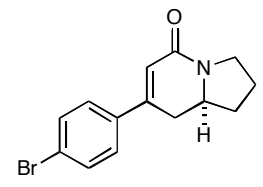

$3 \mathbf{j}$ STANDARd 1H OBSERVE

yu2-305-1

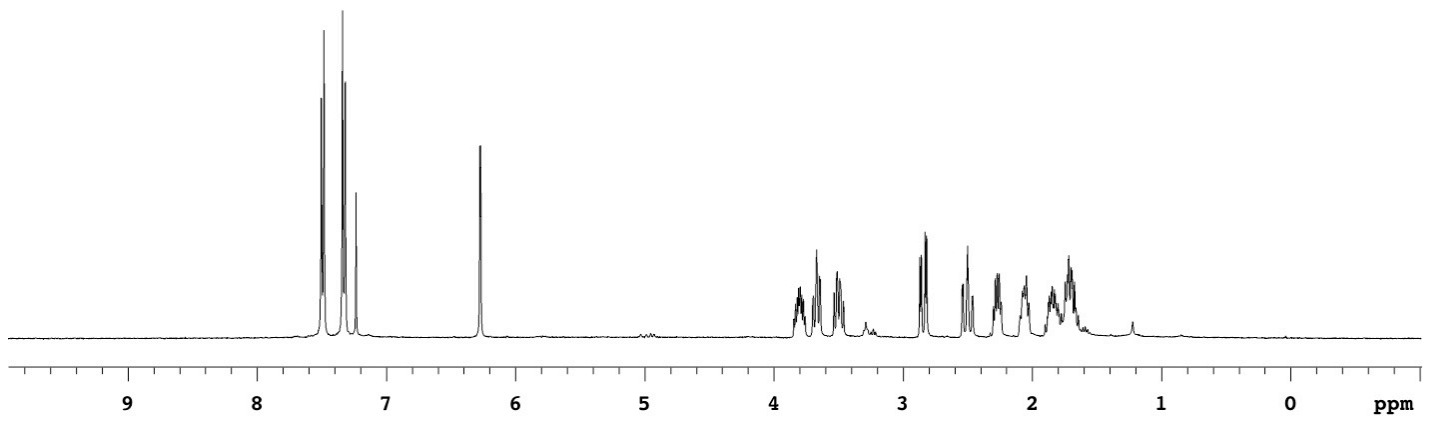

13C OBSERVE

yu2-305-1

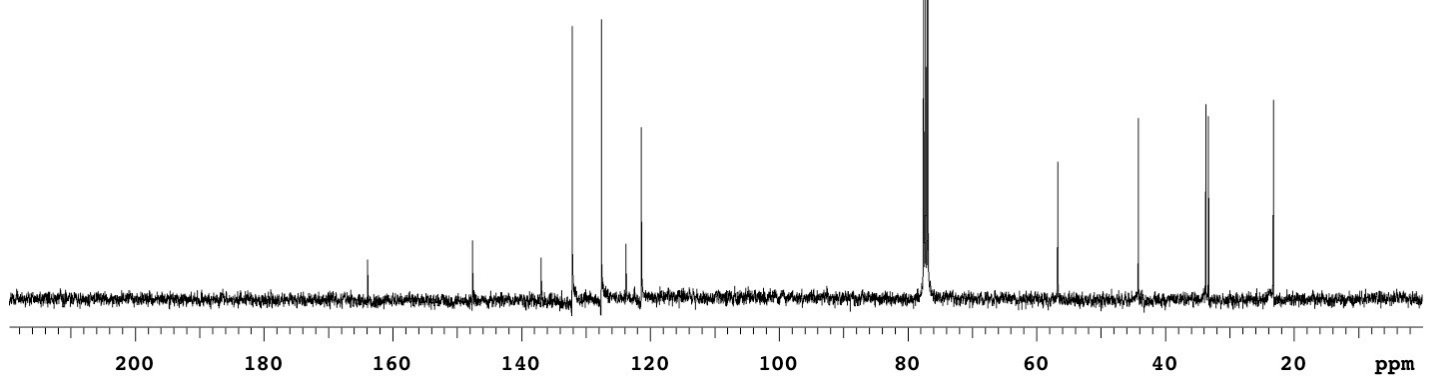




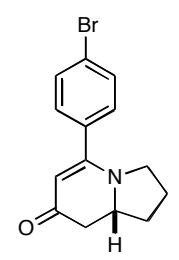

$4 \mathbf{j}$

STANDARD $1 \mathrm{H}$ OBSERvE

yu2-305-2

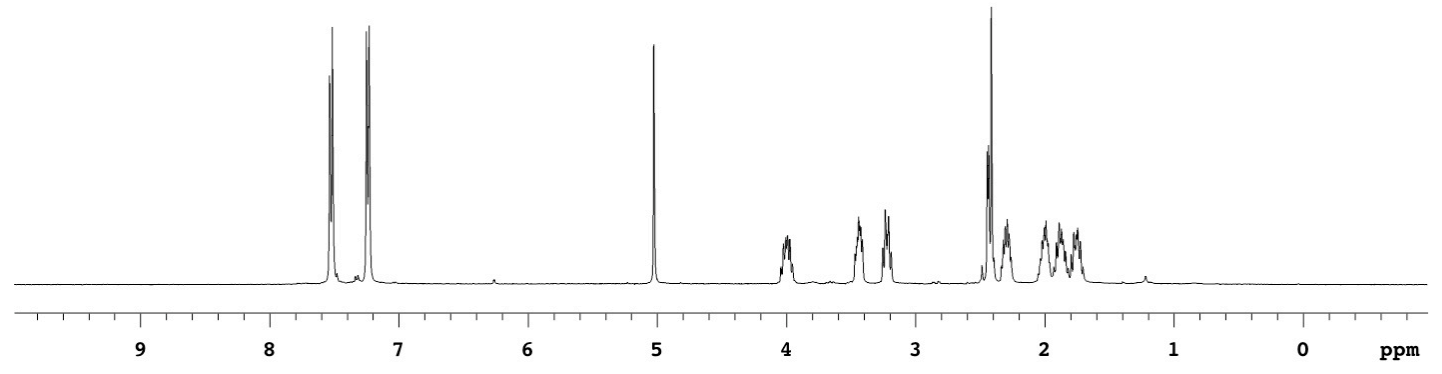

13C OBSERVE

yu2-305-2

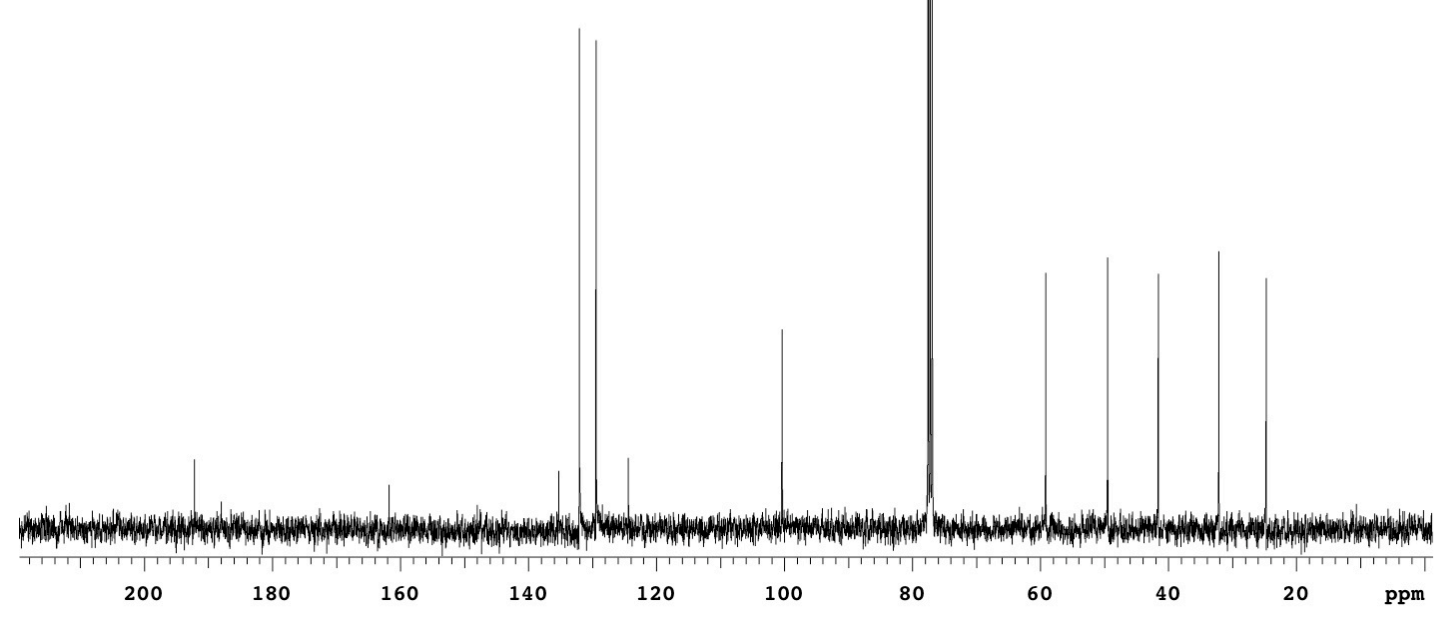

S-26 


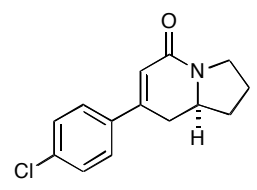

3k

STANDARD 1H OBSERVE

yu2-298-1

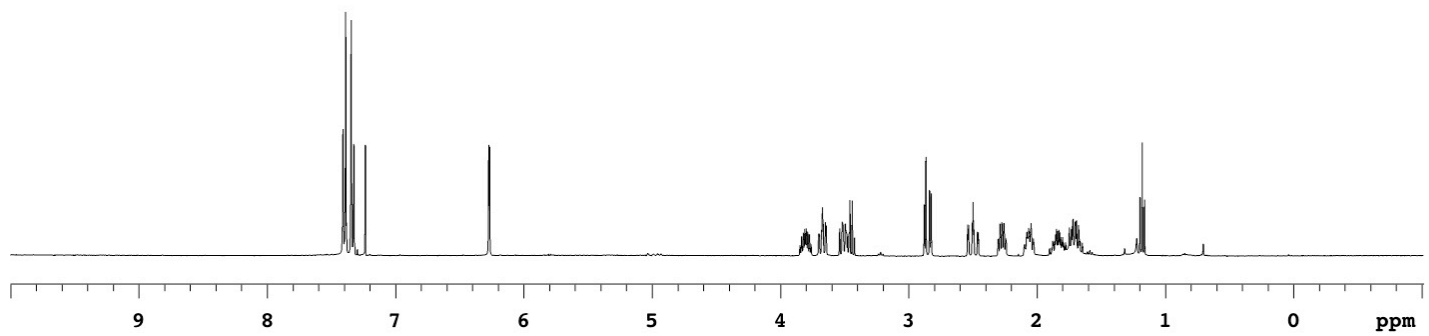

13C OBSERVE

Yu2-298-1

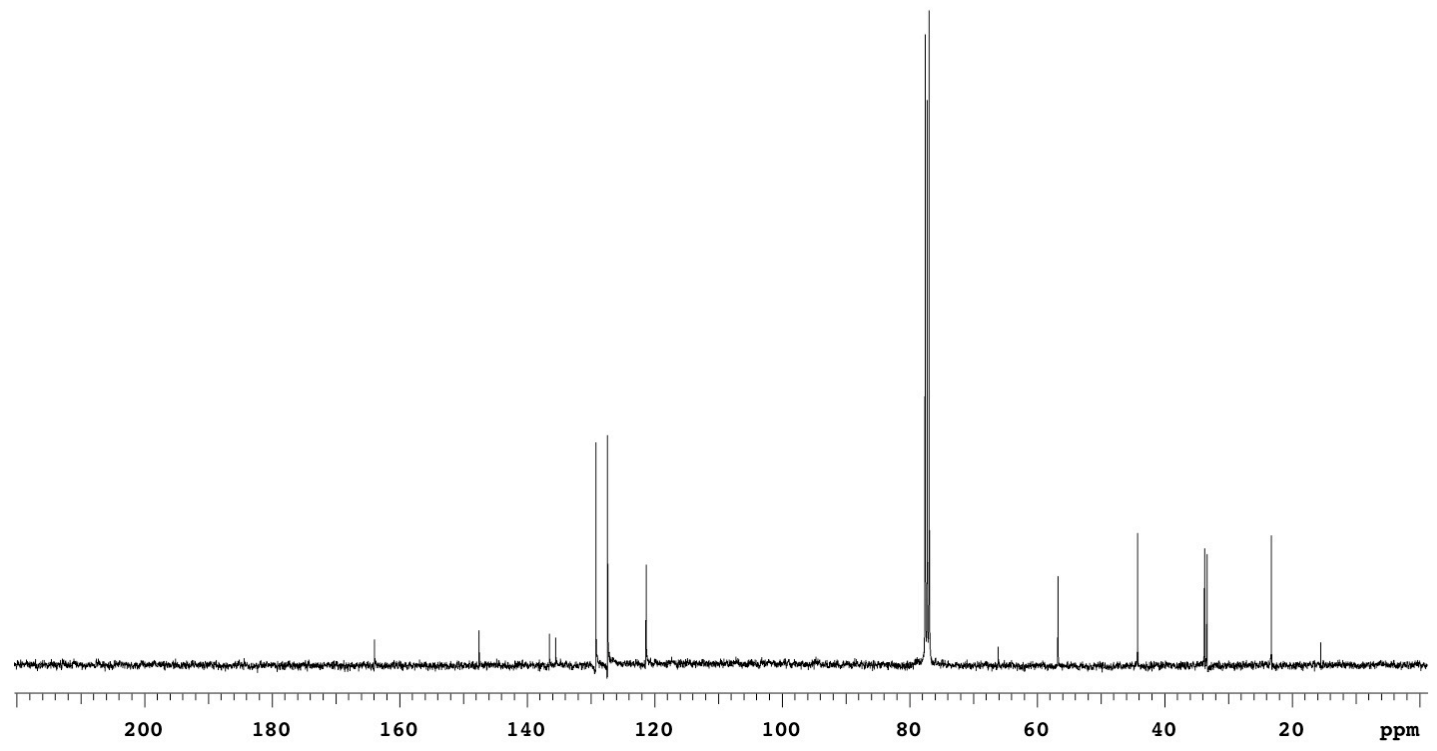




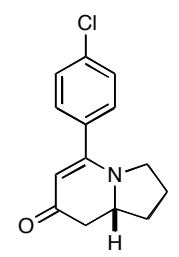

$4 k$

STANDARD 1H OBSERVE

yu2-253-2

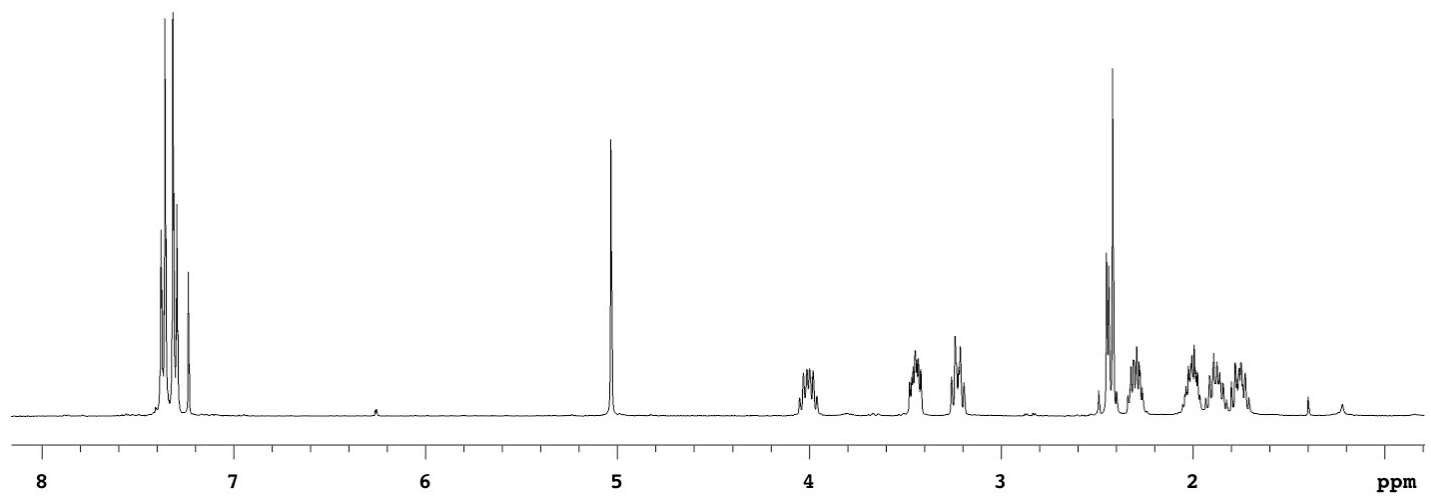

13C OBSERVE

Yu2-253-2

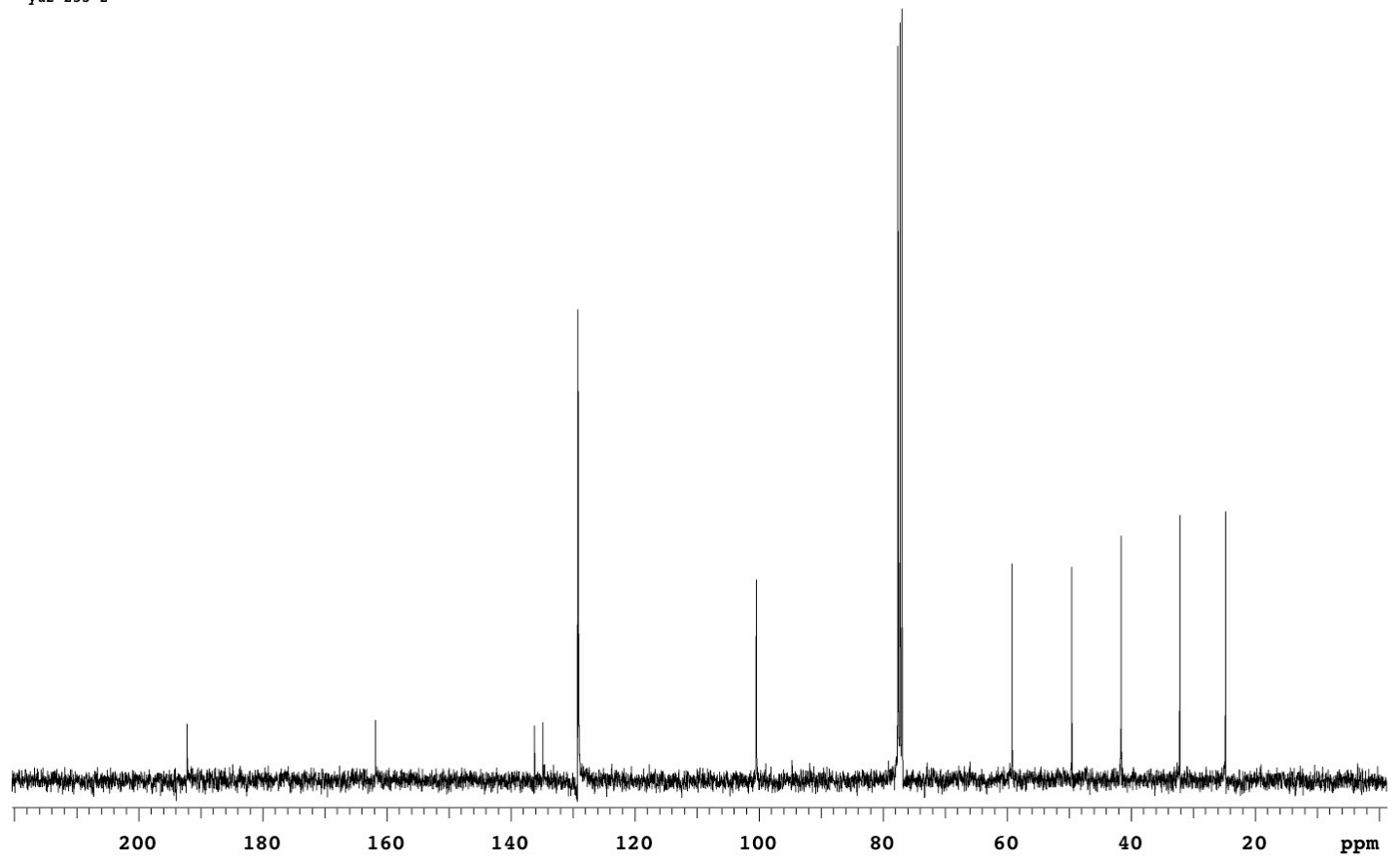

S-28 


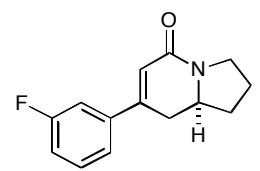

31

yu2-257-1

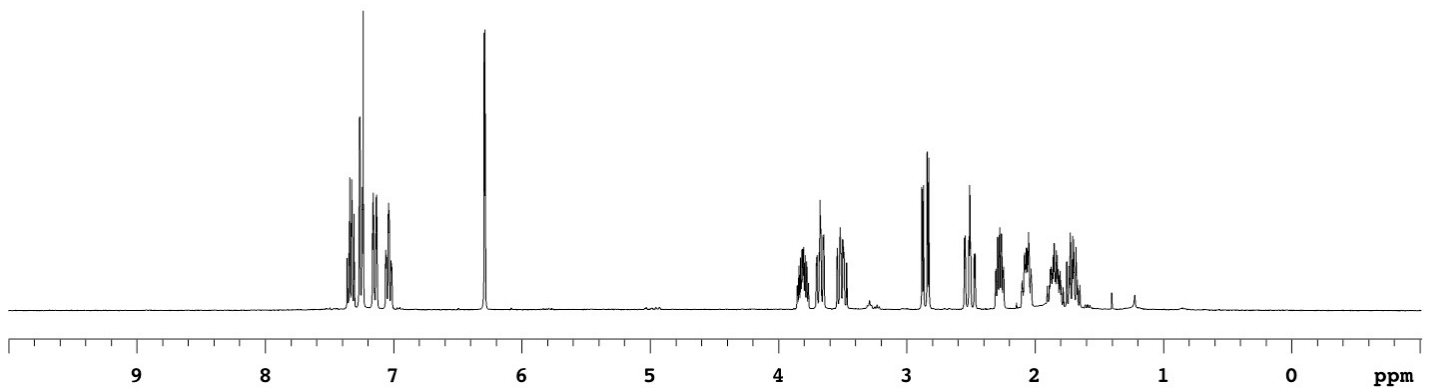

13C OBSERVE

Yu2-257-

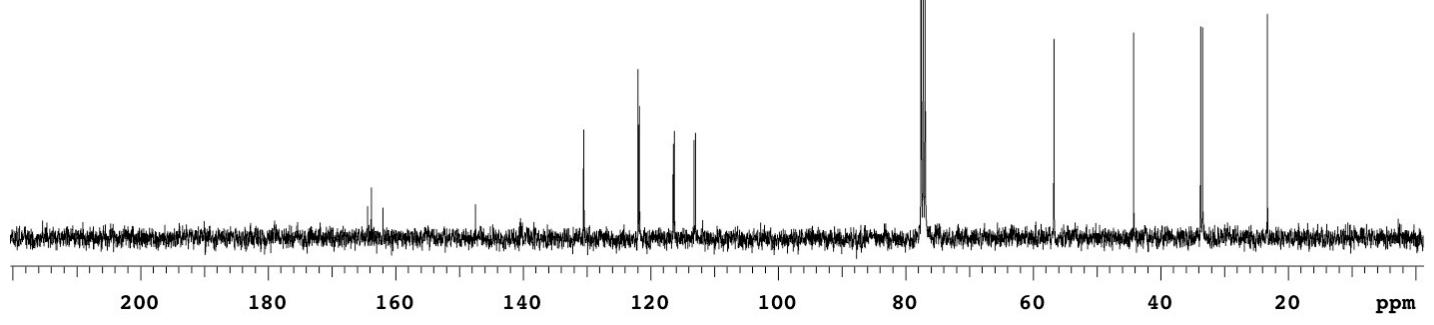




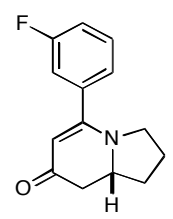

41

STANDARD 1H OBSERVE

yu2-257-2
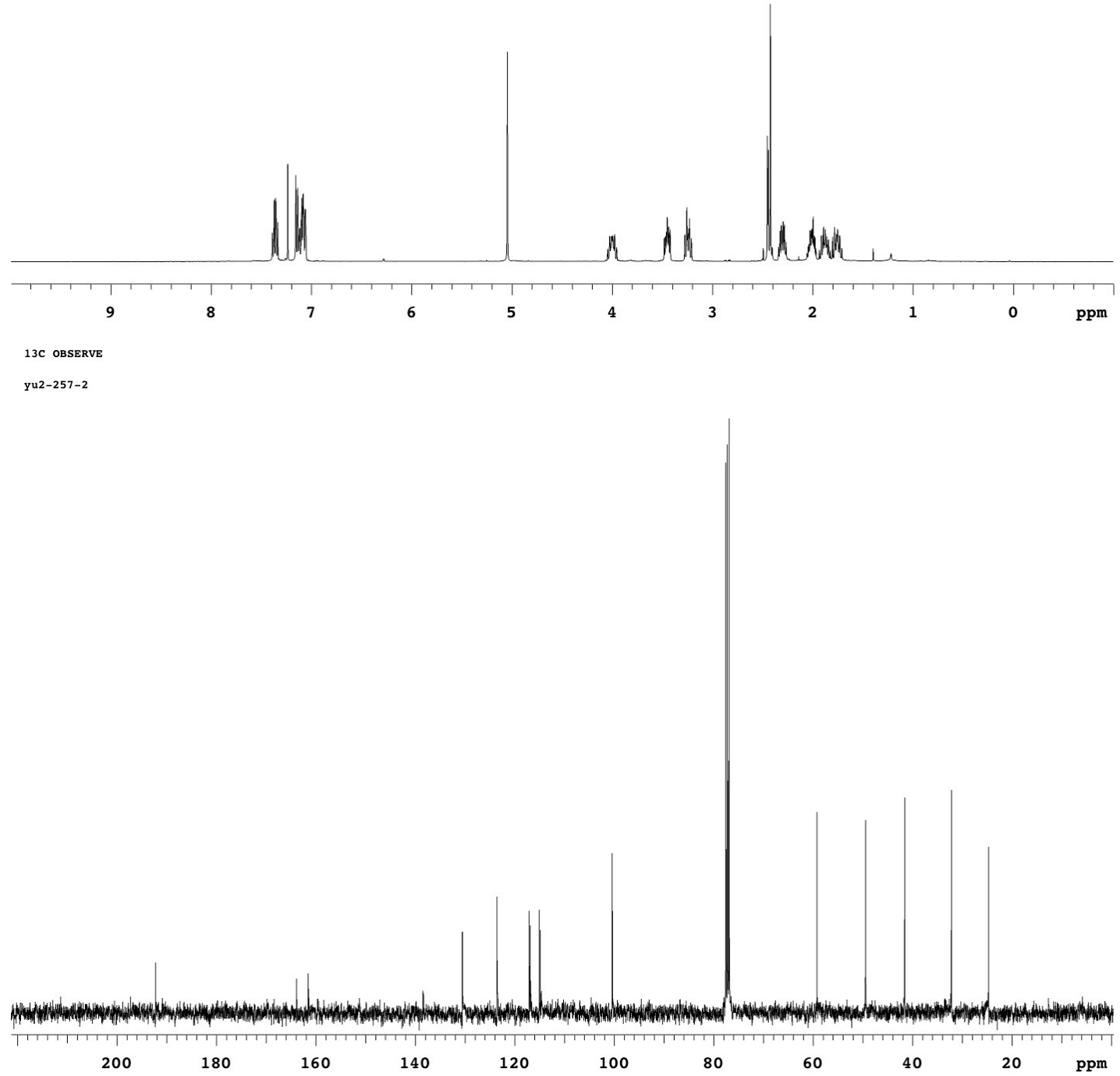

S-30 


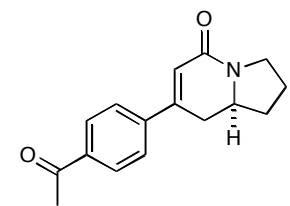

$3 \mathbf{m}$

STANDARD $1 \mathrm{H}$ OBSERVE

yu2-274-1

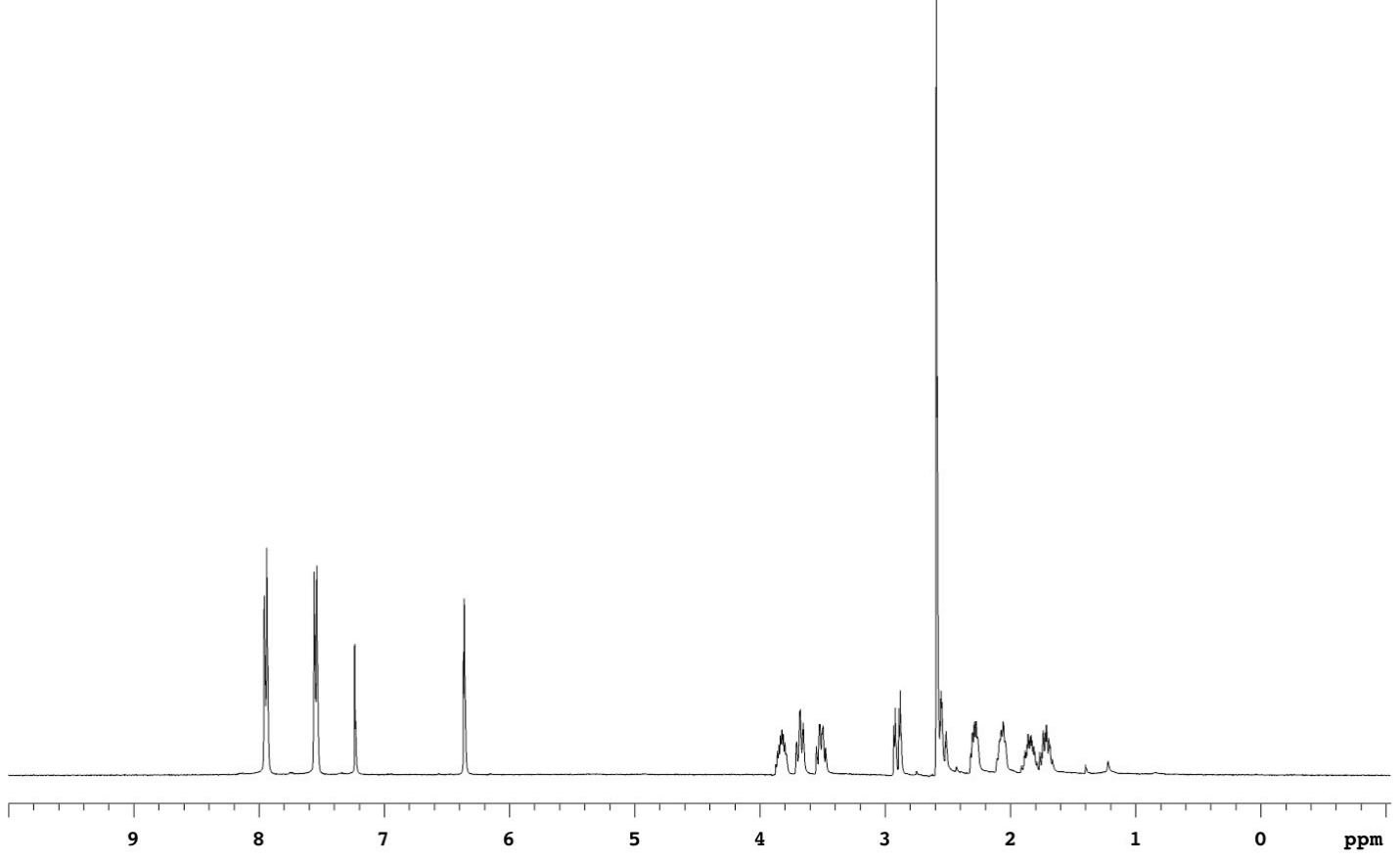

13C OBSERVE

yu2-274-1

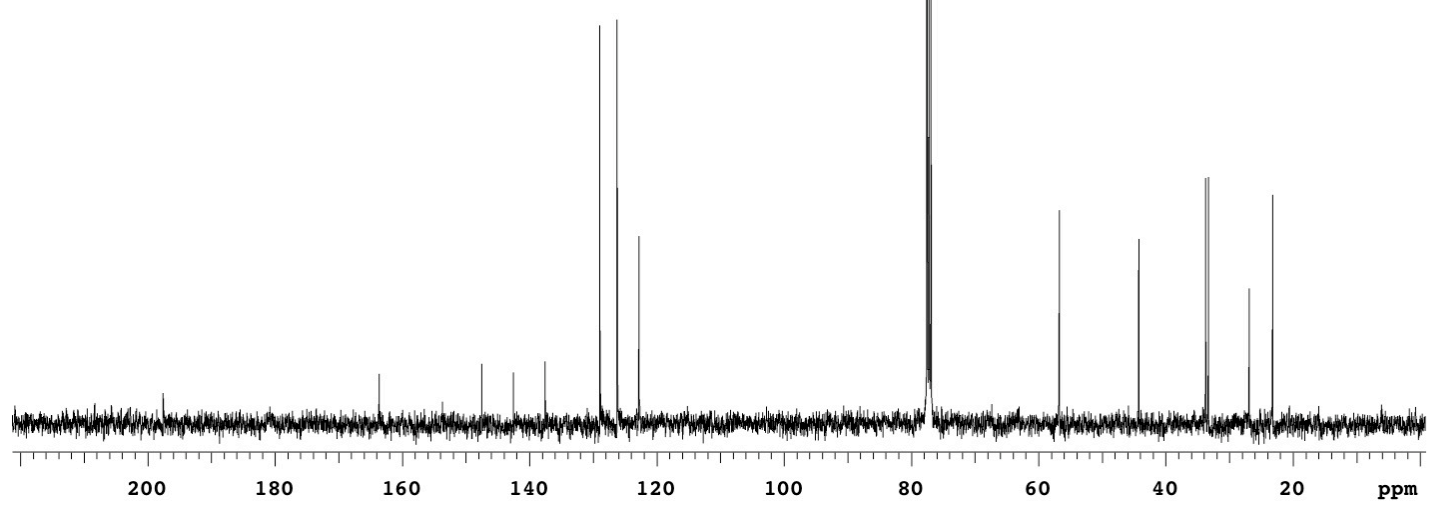




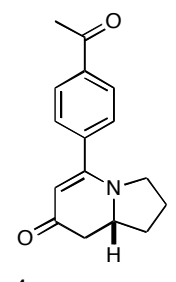

$4 m_{\text {STANDARD } 1 \mathrm{H} \text { OBSERVE }}$

yu2-274-2

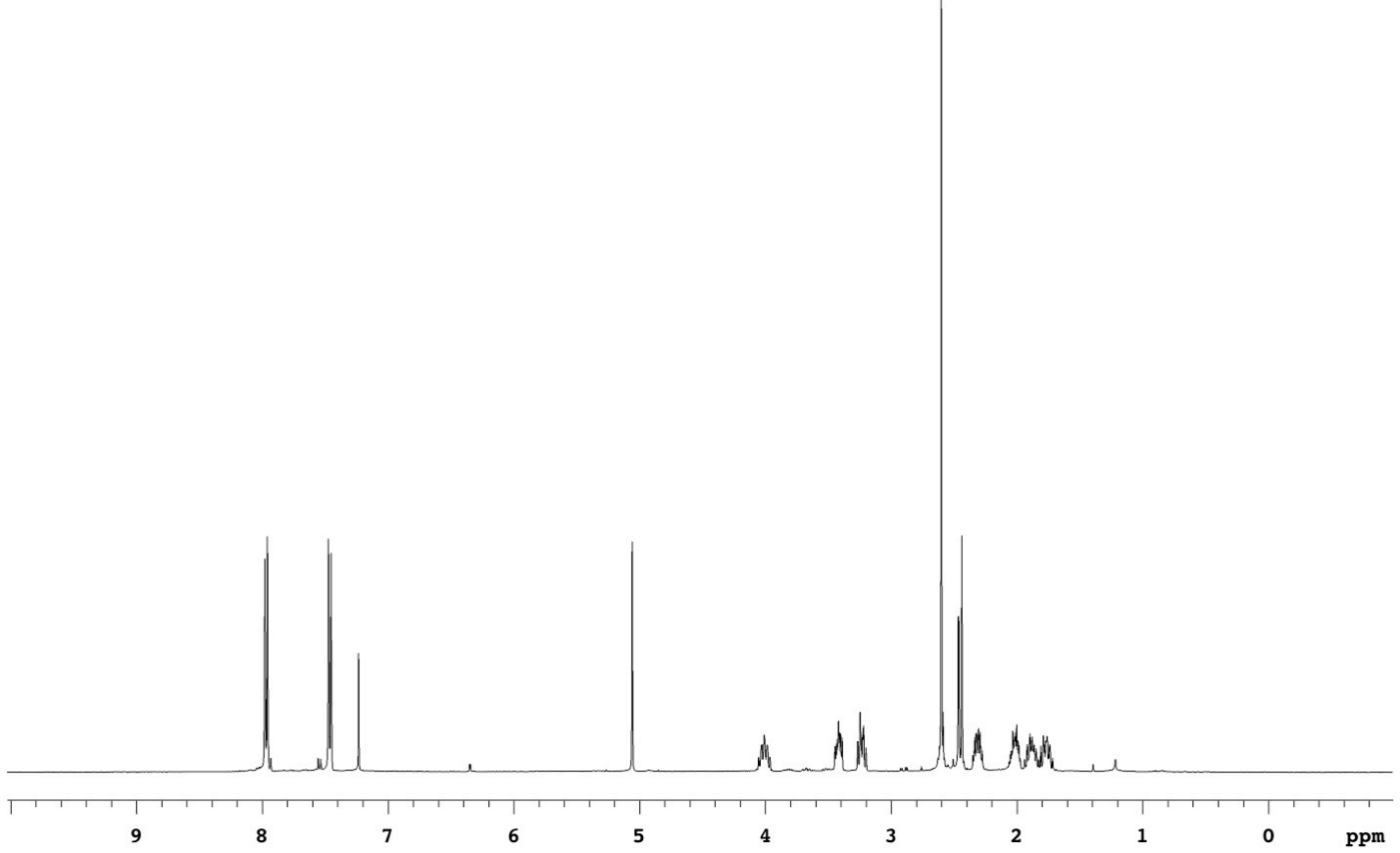

$13 \mathrm{C}$ OBSERVE

yu2-274-2

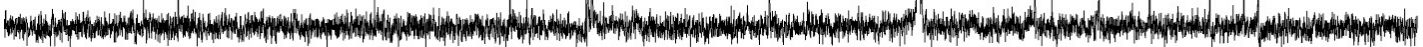

\begin{tabular}{|c|c|c|c|c|c|c|c|c|c|}
\hline 200 & 180 & 160 & 140 & 120 & 100 & 80 & 60 & 40 & 20 \\
\hline
\end{tabular}

S-32 


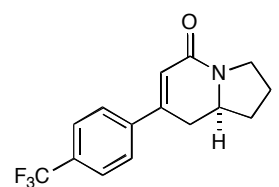

3n

STANDARD 1H OBSERVE

yu2-276-1

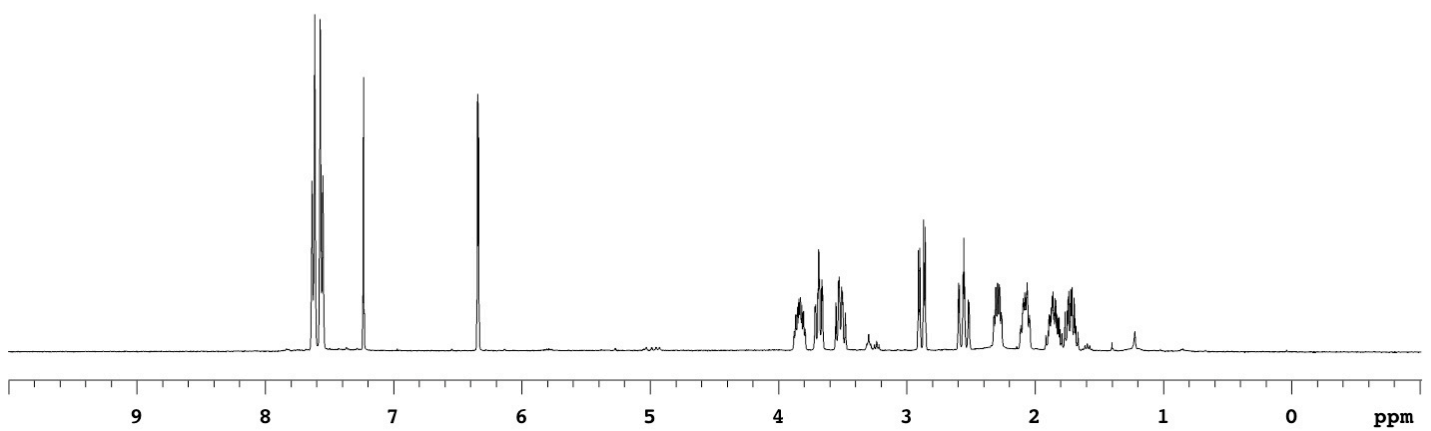

13C OBSERVE

yu2-276-1

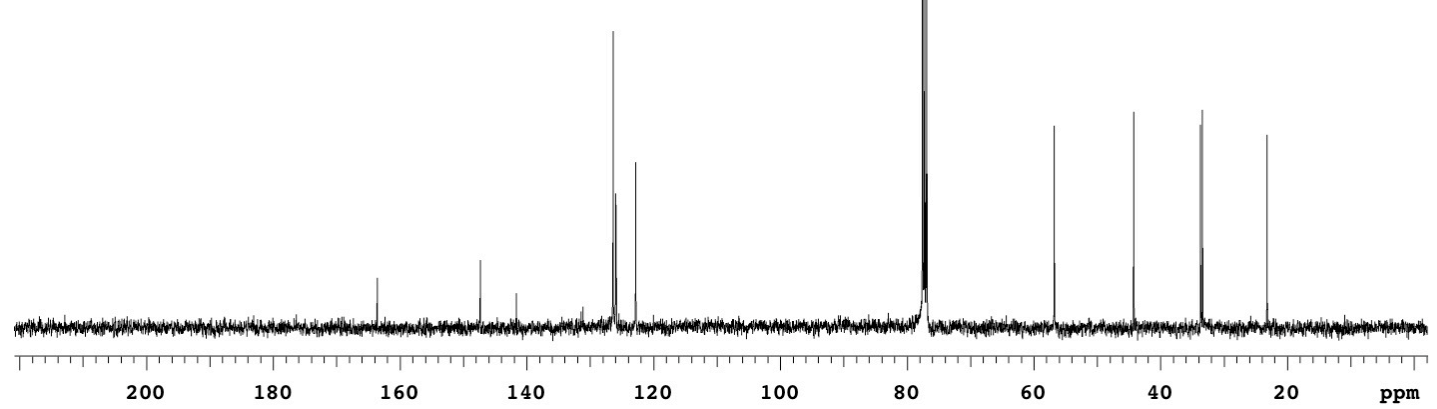

S-33 


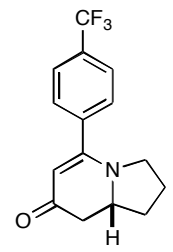

$4 n$

STANDARD 1H OBSERVE

yu2-276-2

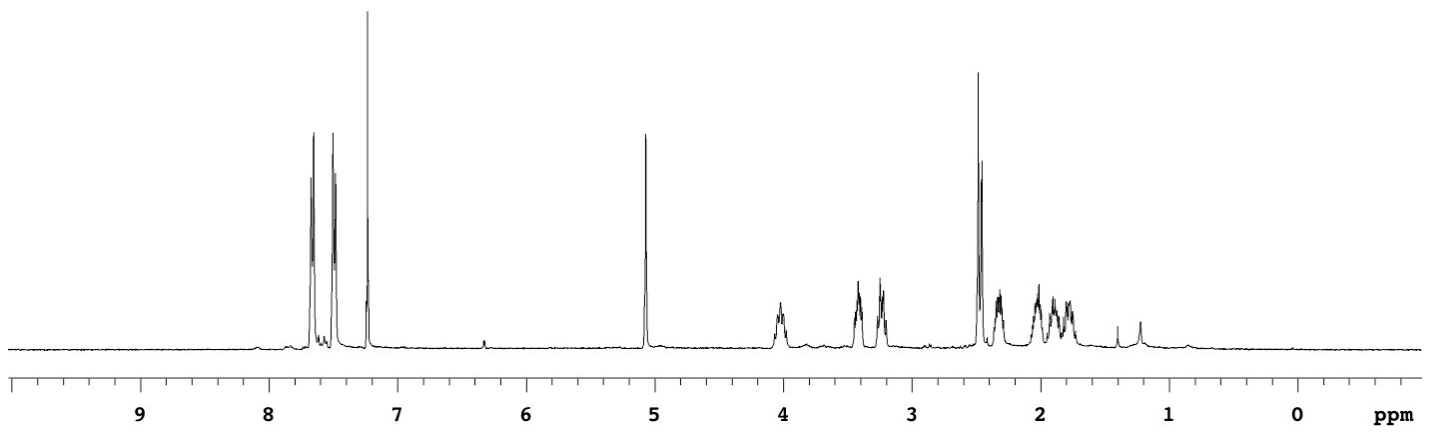

13C OBSERVE

yu2-276-2

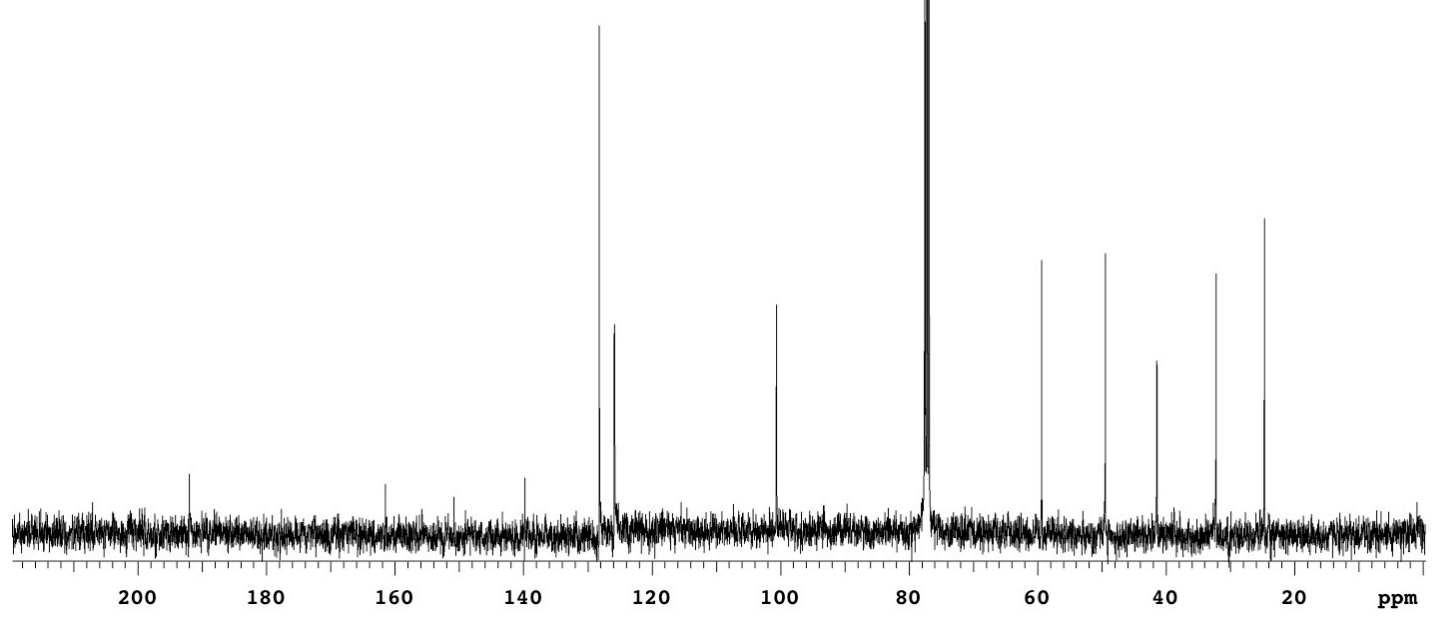

S-34 


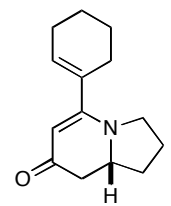

40

STANDARD $1 \mathrm{H}$ OBSERVE

yu2-303
yu2-303

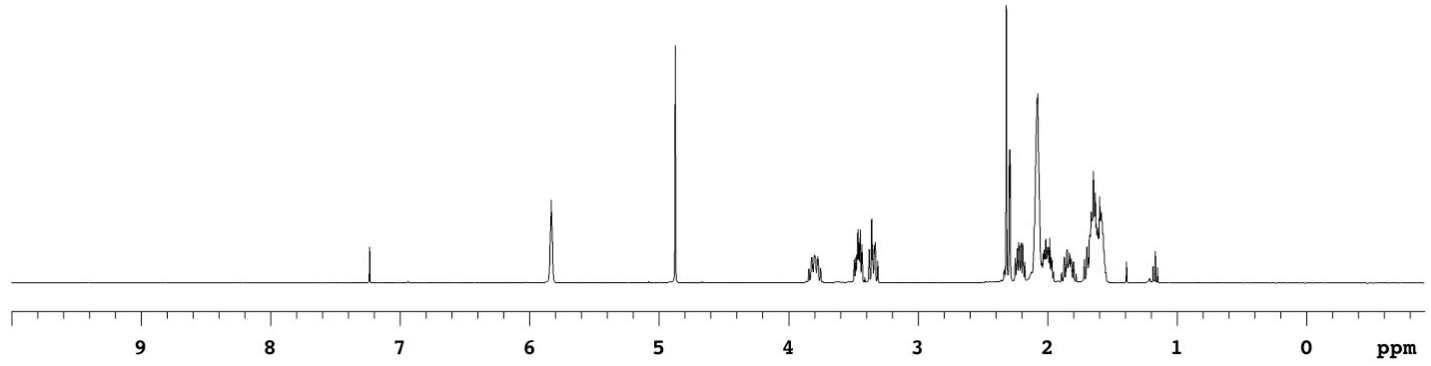

13C OBSERVE

yu2-303

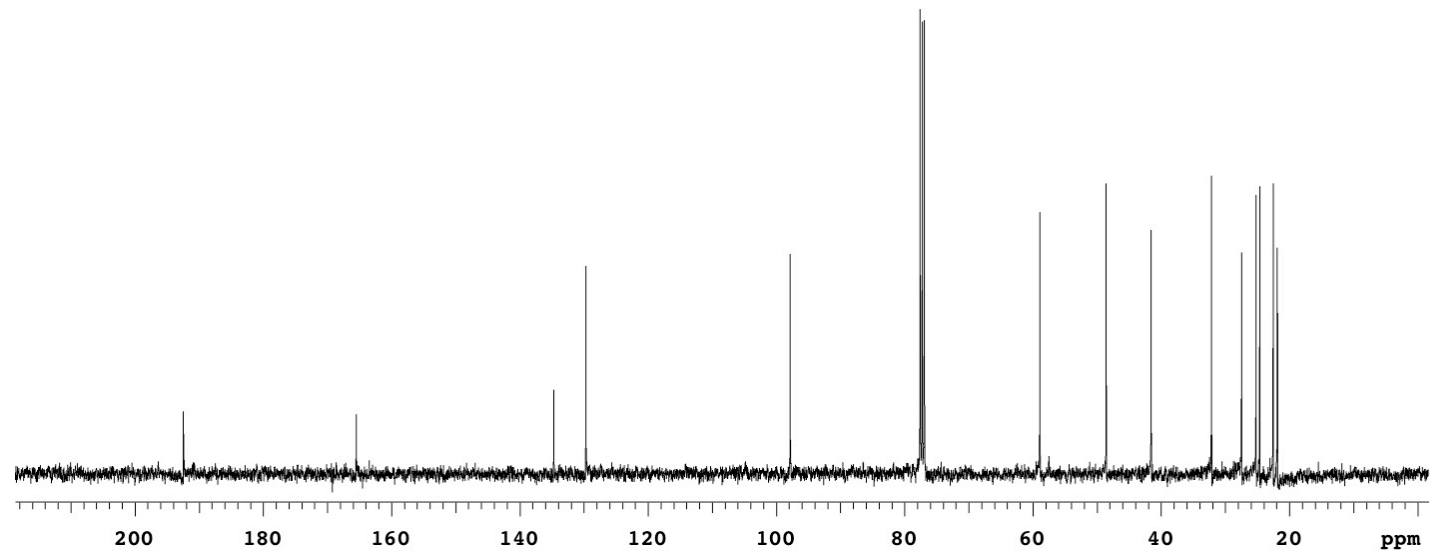

S-35 


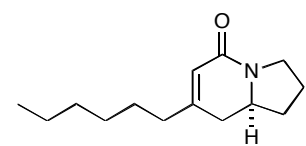

$\mathbf{3 p}_{\text {STANDARD 1H OBSERVE }}$

yu2-208-1
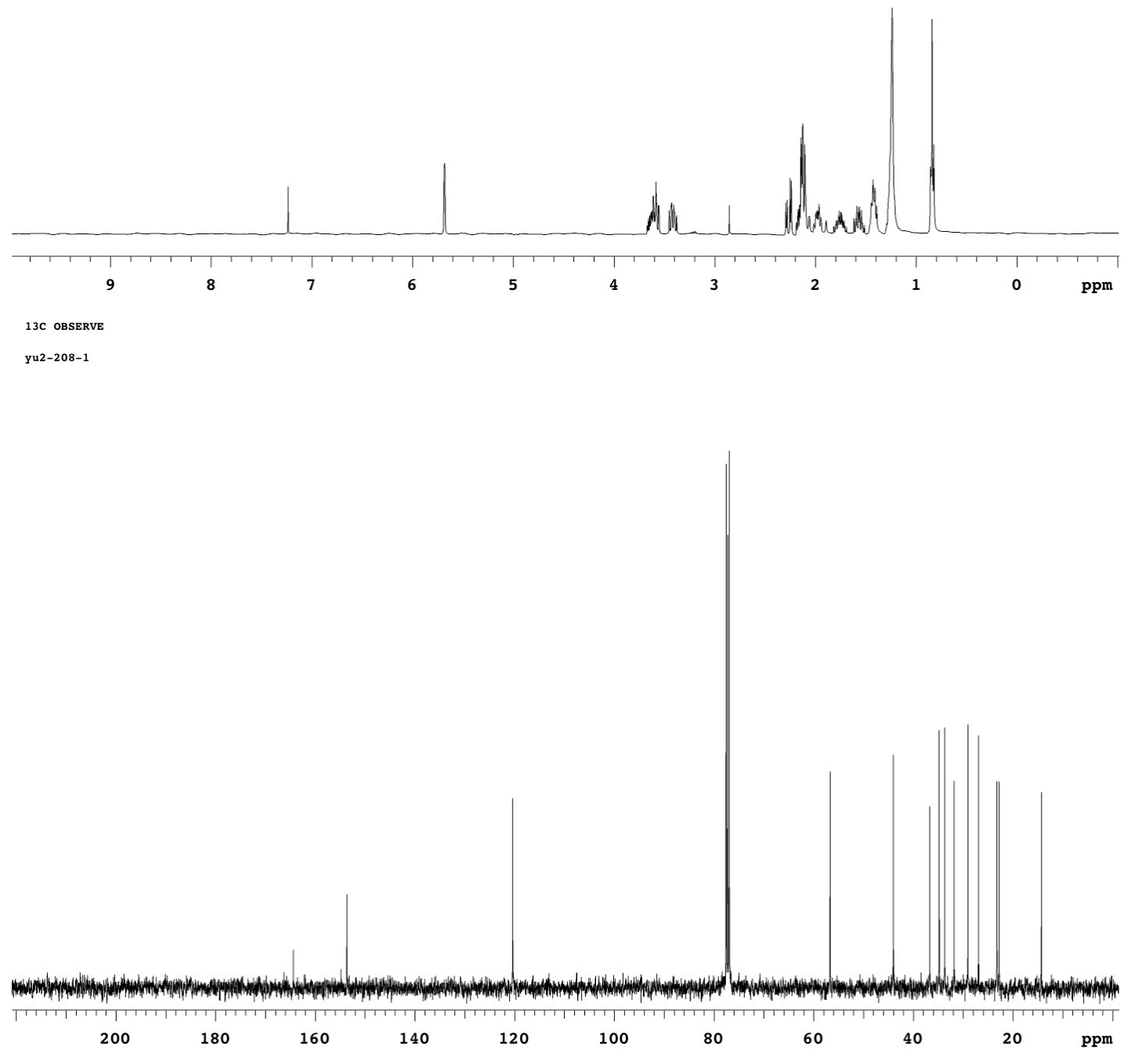

S-36 


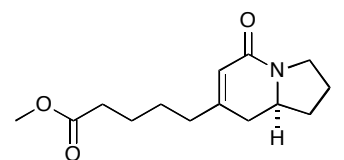

$\mathbf{3 q}_{\text {STANDARD 1H OBSERVE }}$

yu2-255

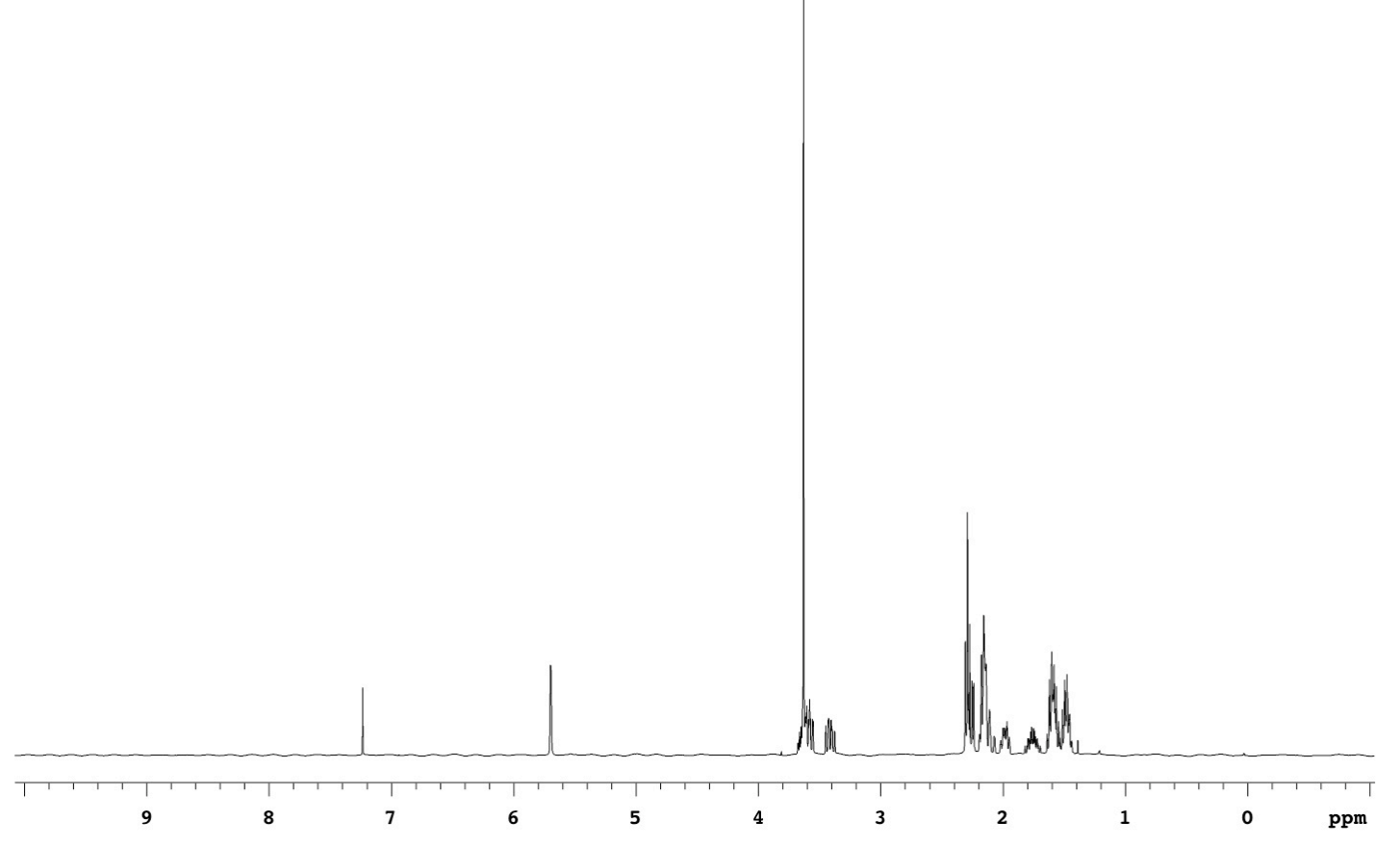

13C OBSERVE

yu2-255-1

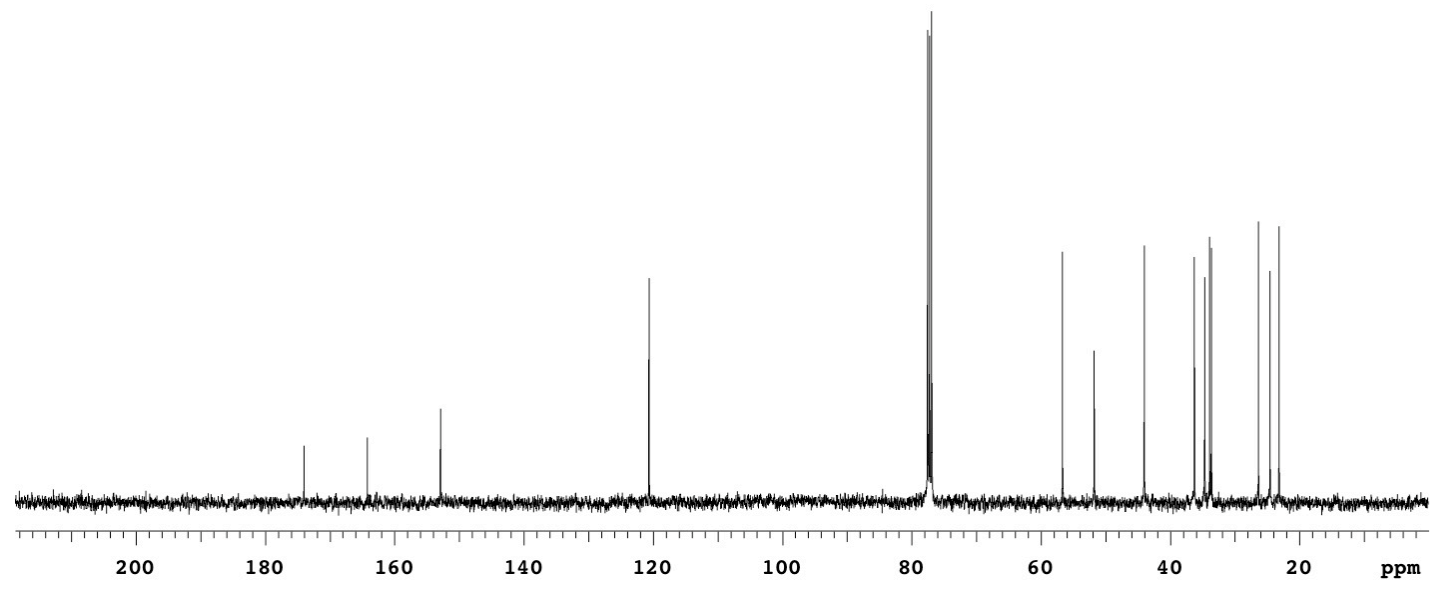




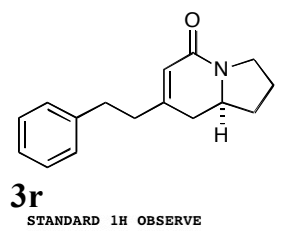

yu2-215-1
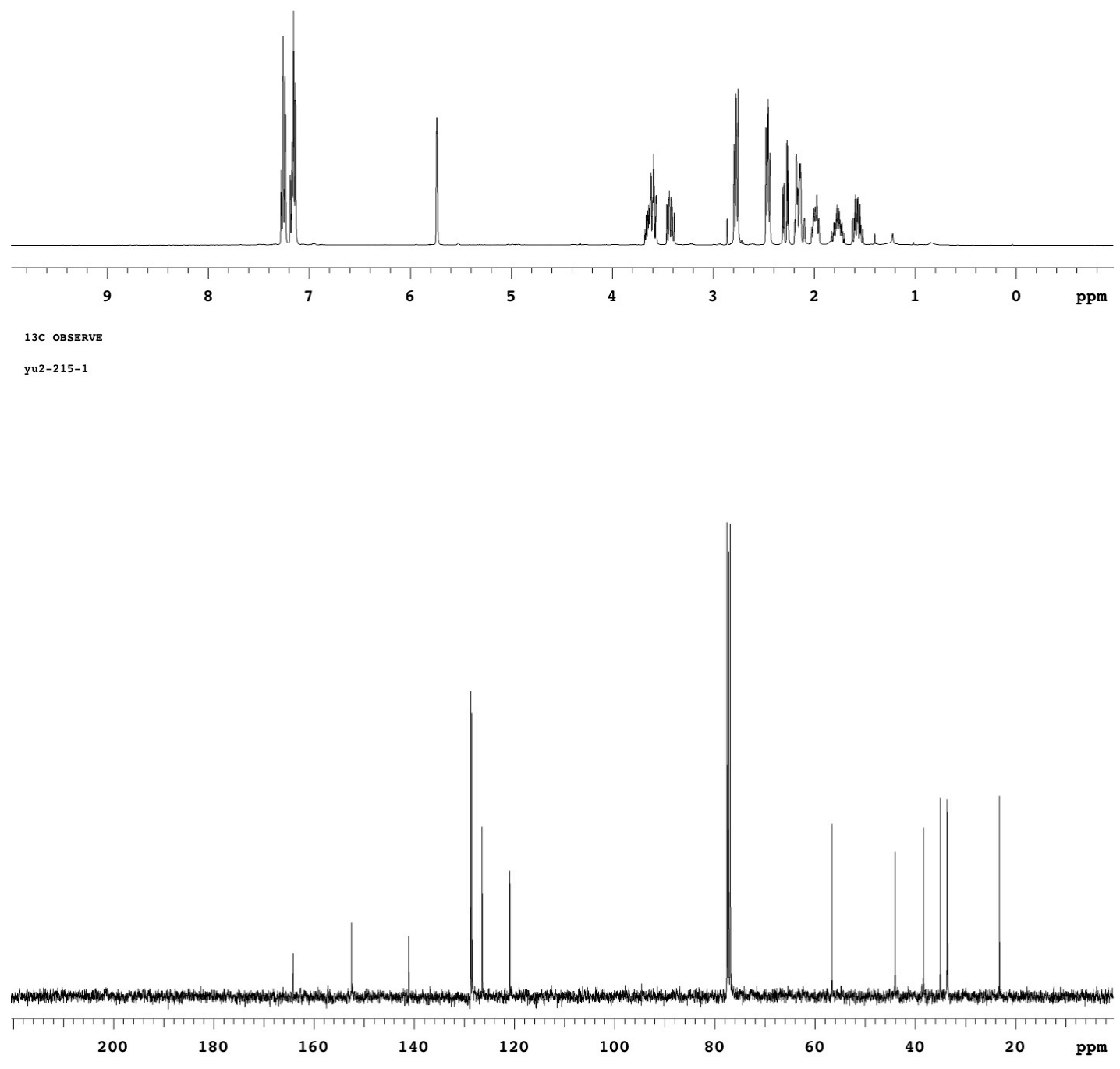


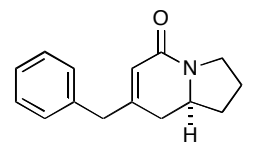

$3 s$

STANDARD 1H OBSERVE

yu2-212-1

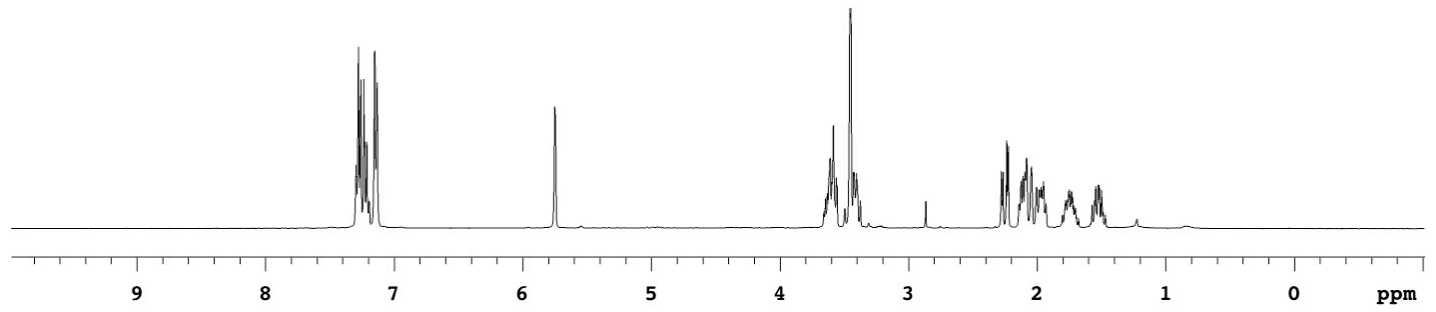

13C OBSERVE

yu2-212-1

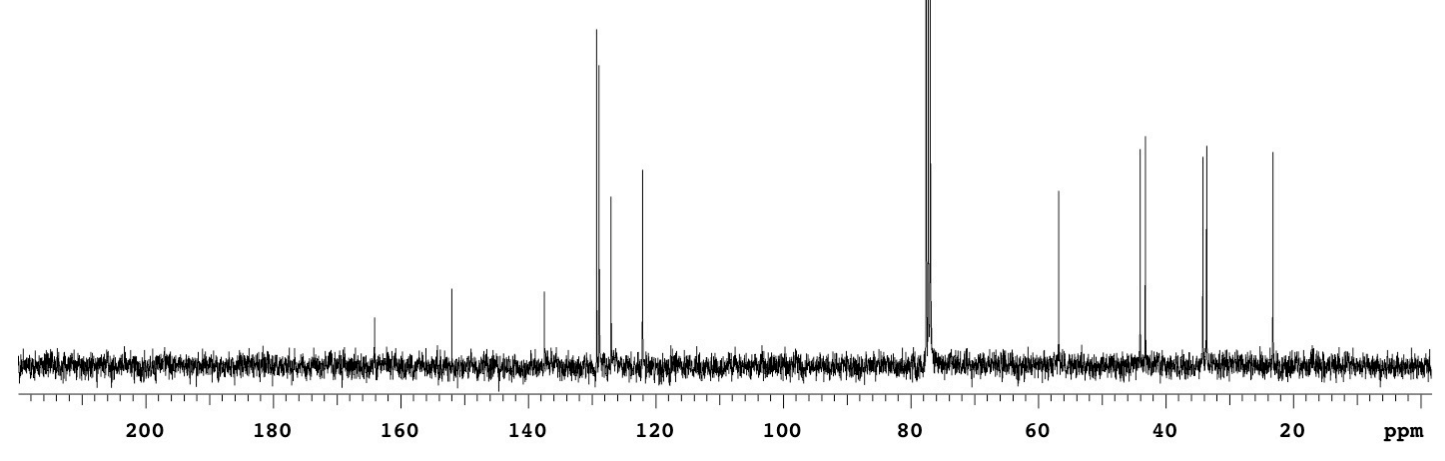




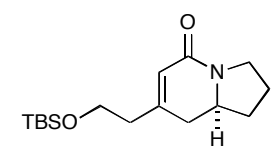

$3 t$

STANDARD $1 \mathrm{H}$ OBSERVE

yu2-214-1

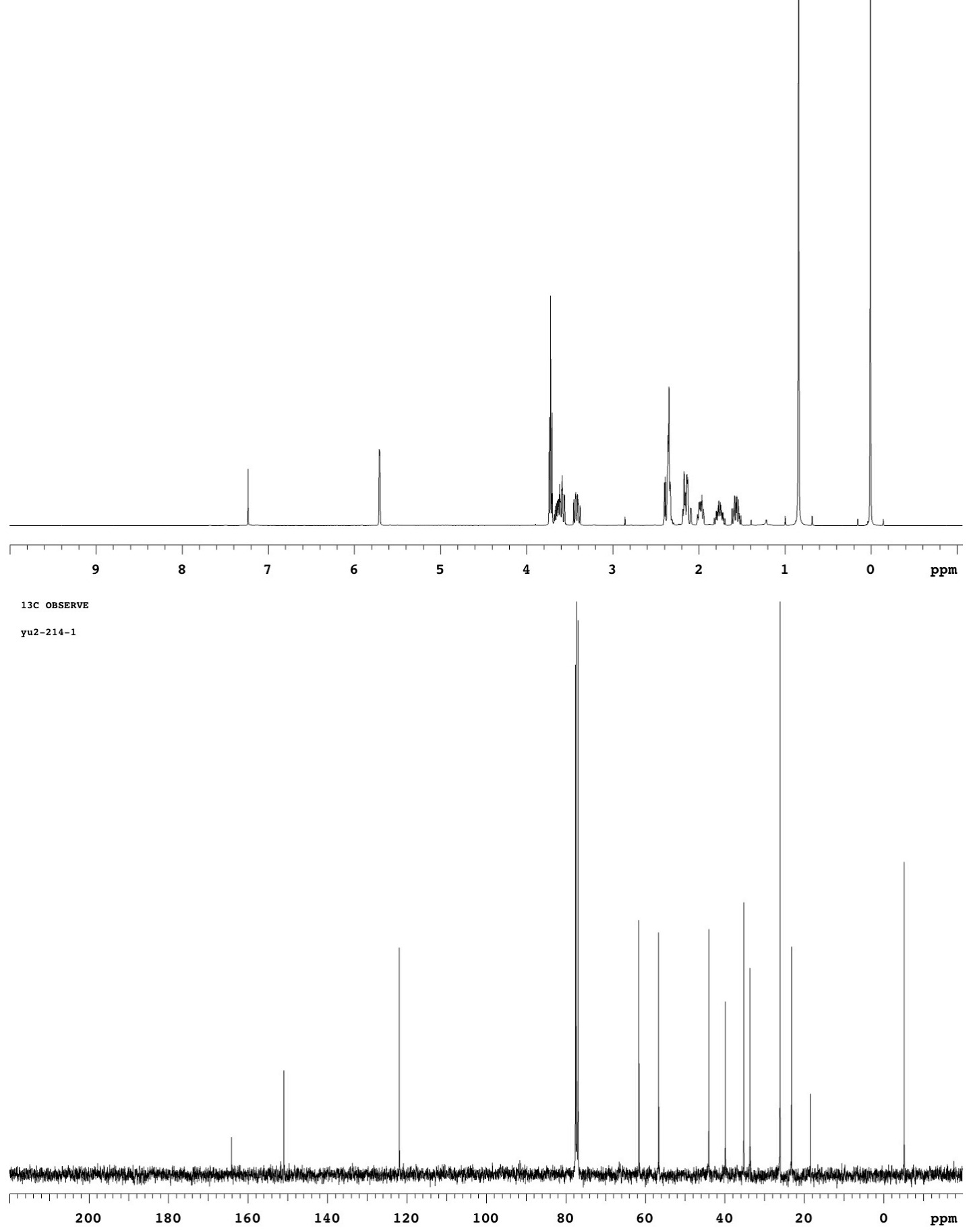




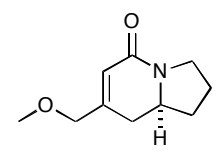

$3 \mathbf{u}$

STANDARD $1 \mathrm{H}$ OBSERVE

Yu2-290

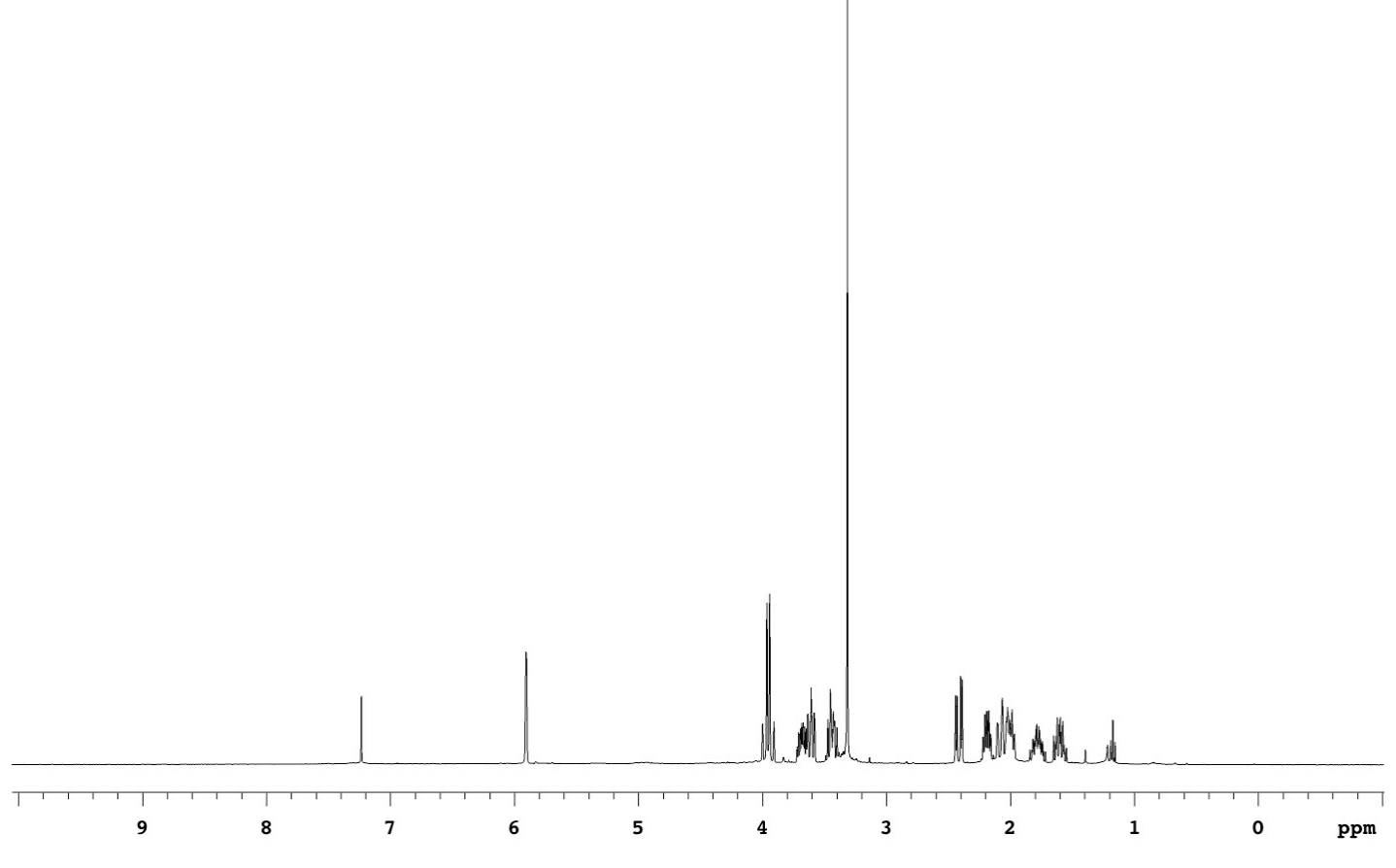
13C OBSERVE

уи2-290

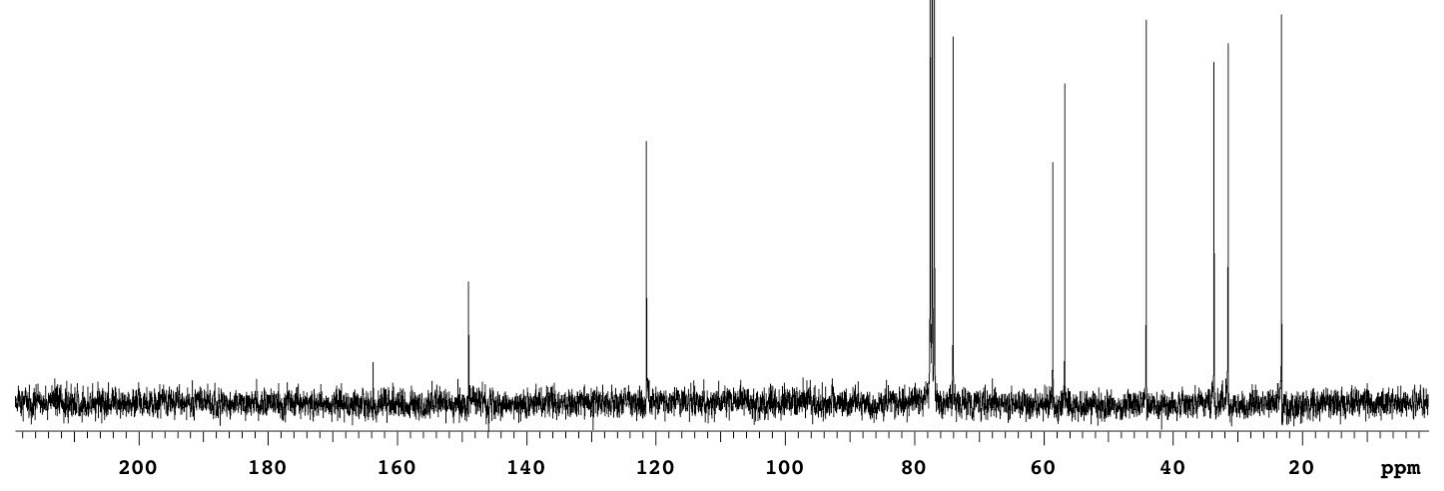




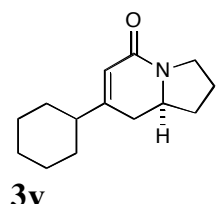

STANDARD $1 \mathrm{H}$ OBSERVE

yu2-211-1

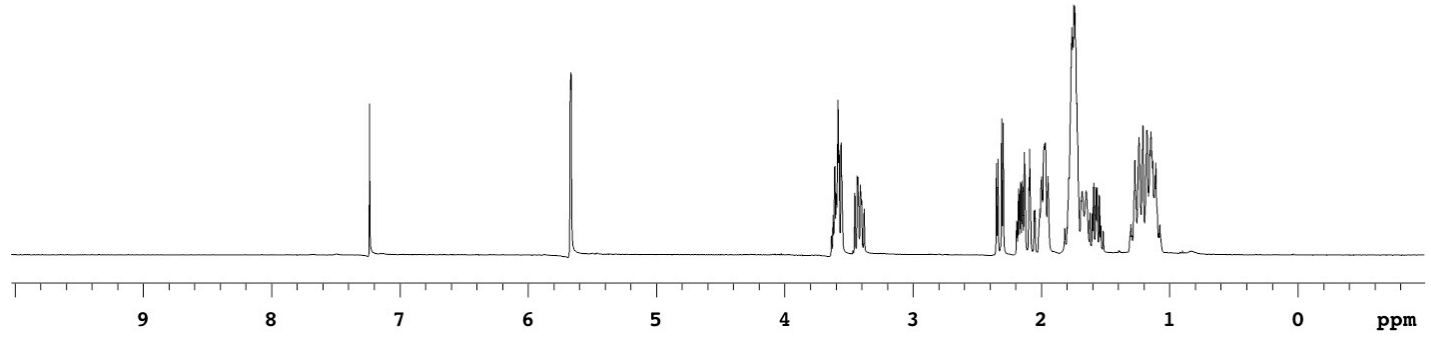

13C OBSERVE

yu2-211-1

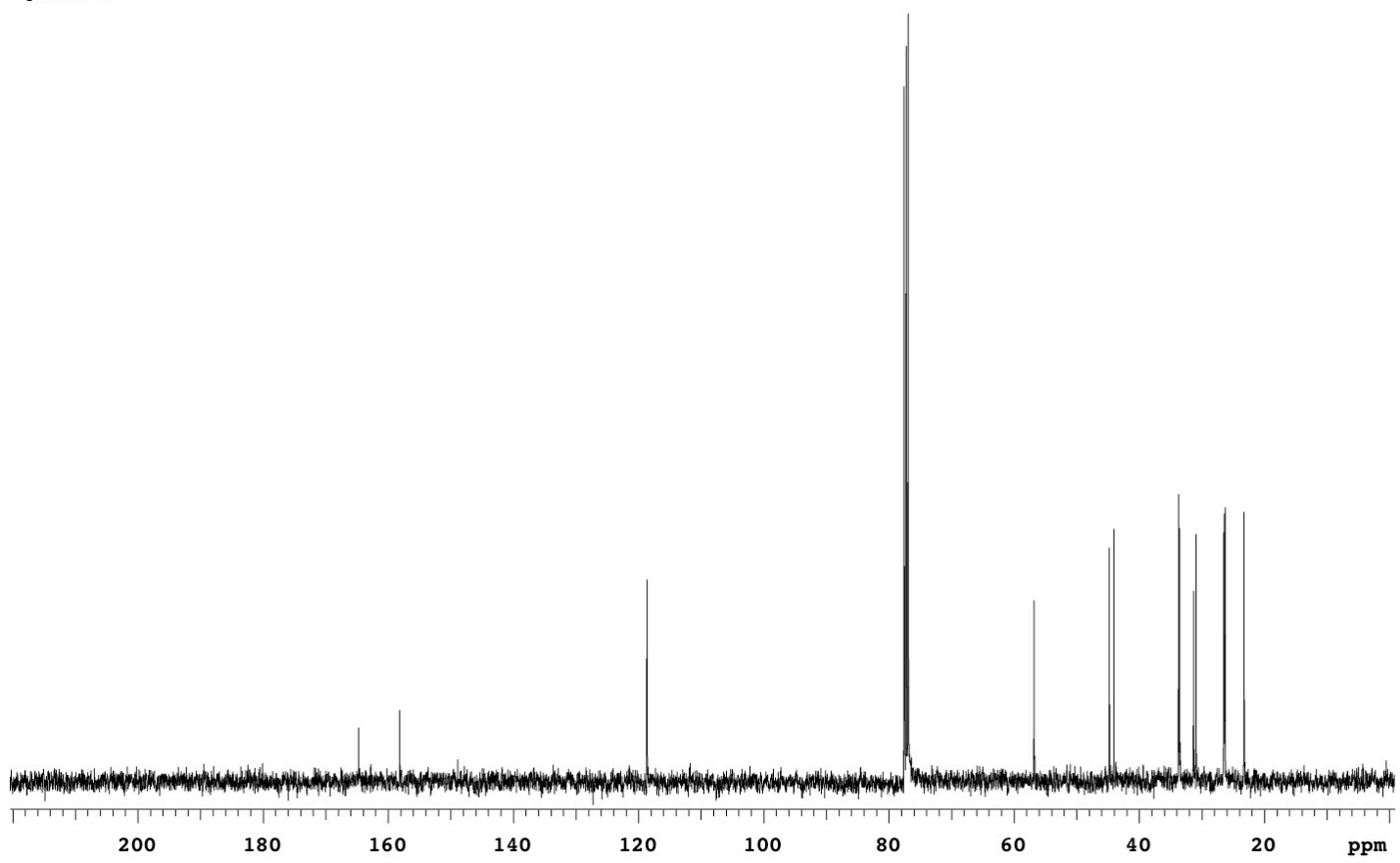




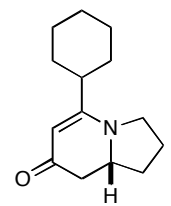

4v

STANDARD 1H OBSERVE

yu2-211-2

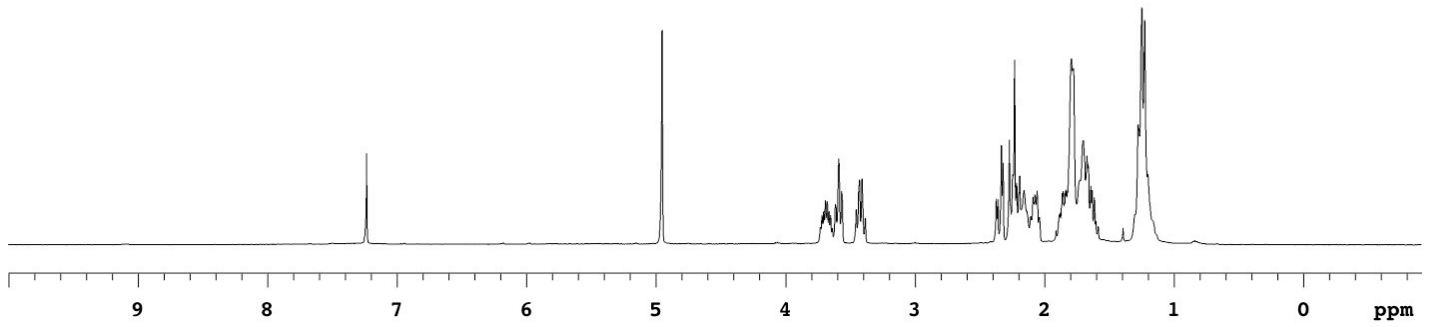
13C OBSERVE

yu2-211-2

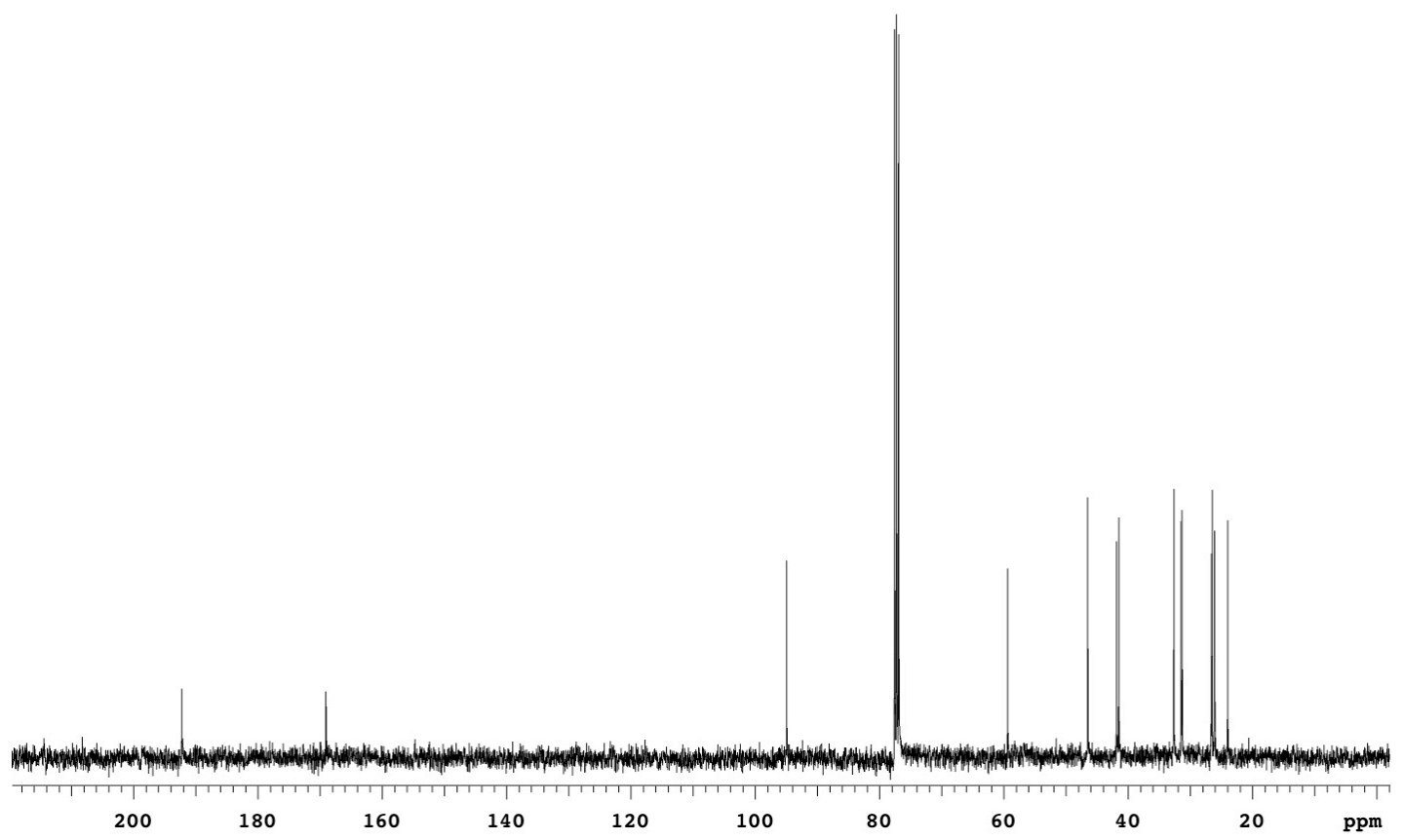




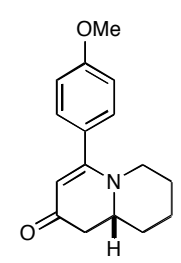

6c

STANDARD 1H OBSERVE

yu2-261-2

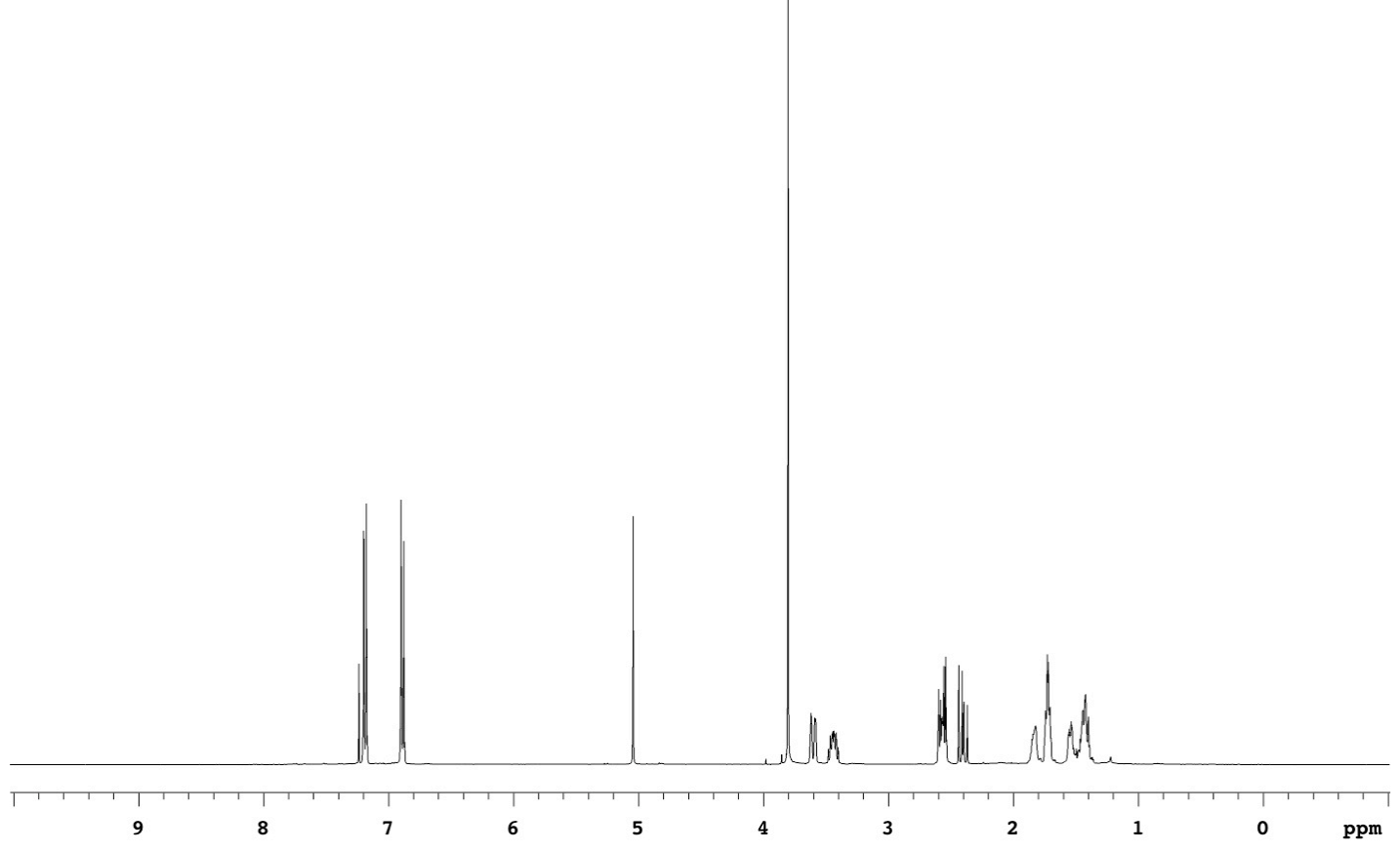

13C OBSERVE

yu2 2 -261-2
yu2-261-2

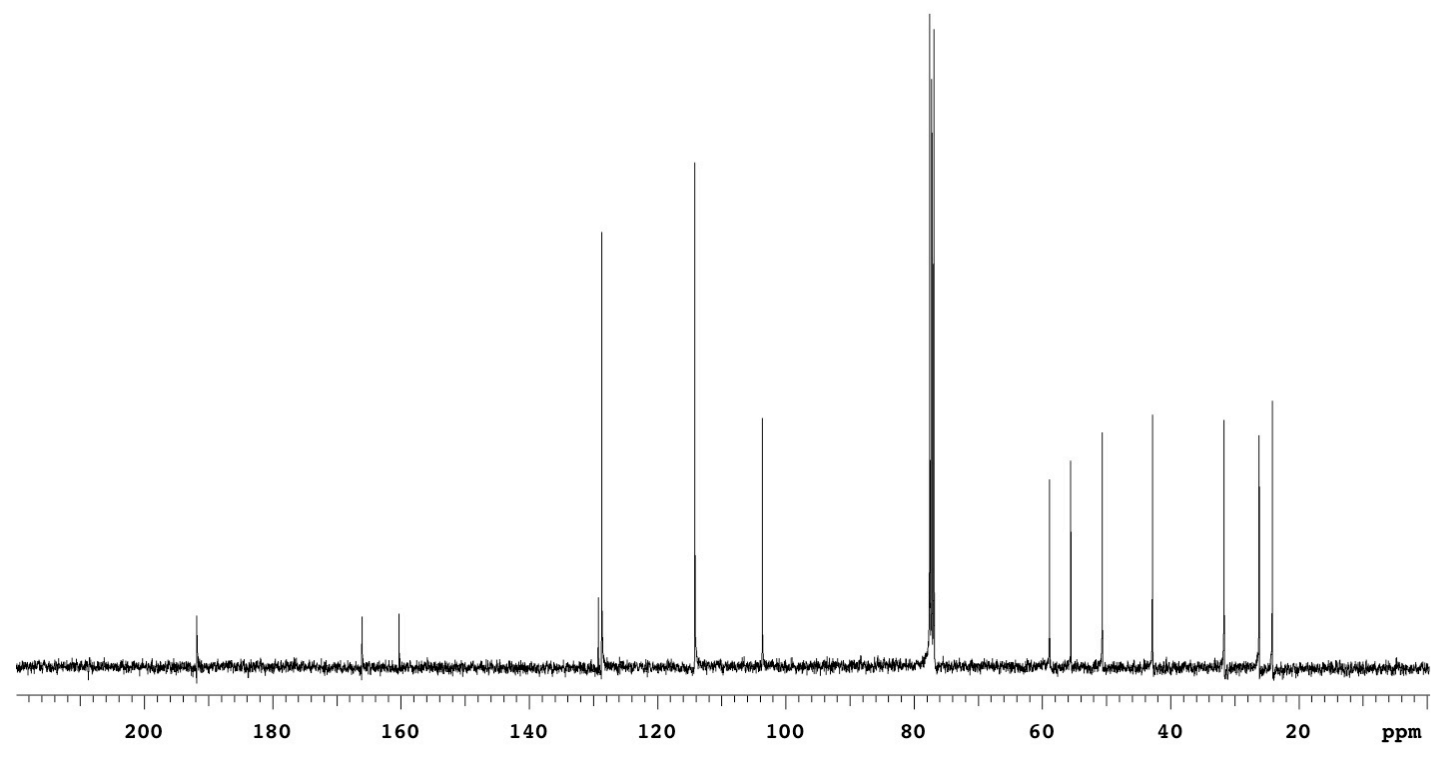




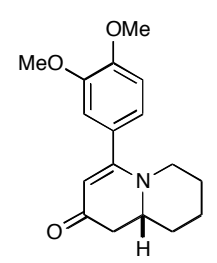

6b

STANDARD $1 \mathrm{H}$ OBSERVE

yu2-279-2

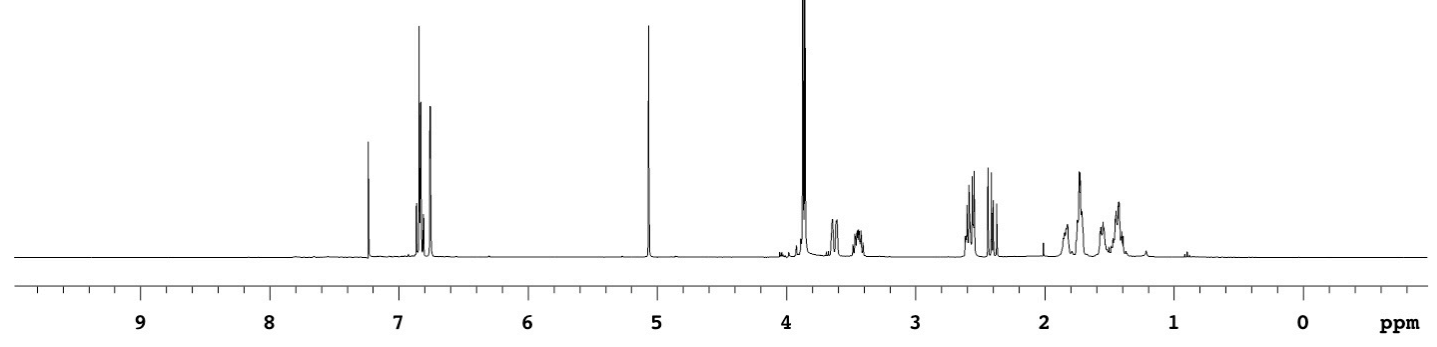

13C OBSERVE

yu2-279-2

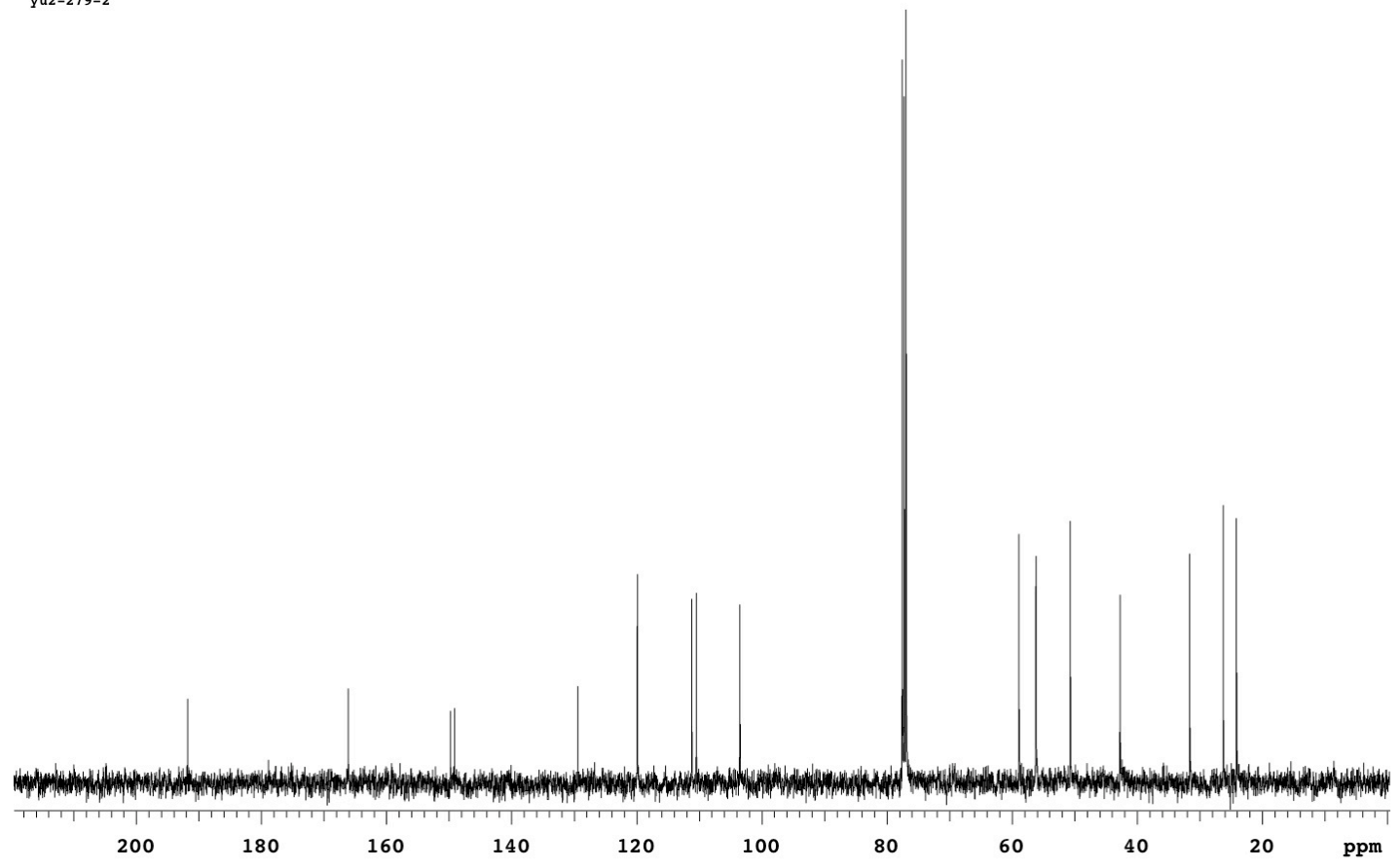




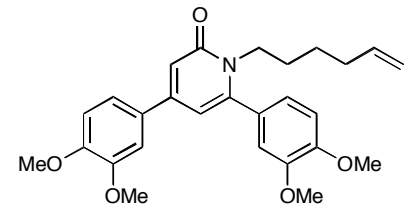

7

STANDARD $1 \mathrm{H}$ OBSERVE

Yu2-297-1

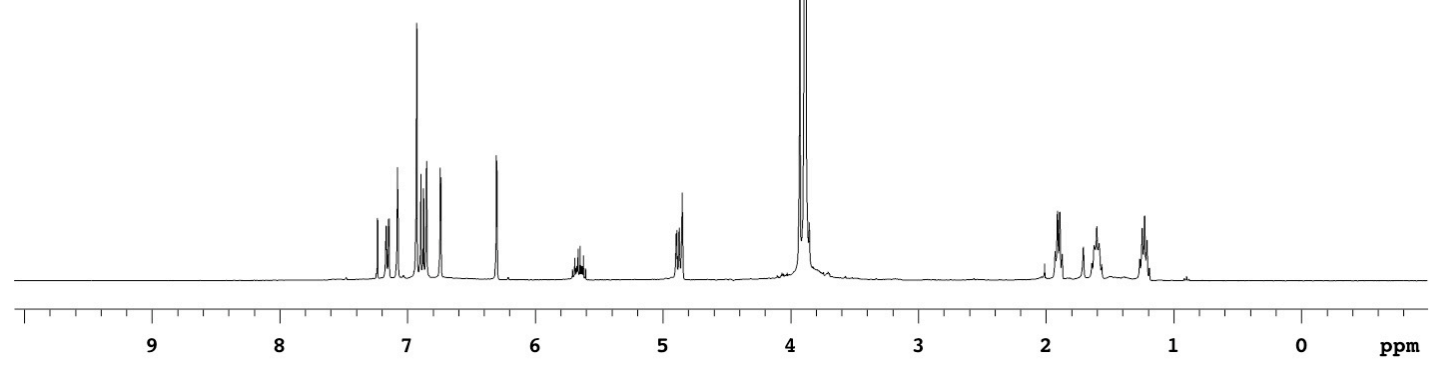

13C OBSERVE

yu2-297-1

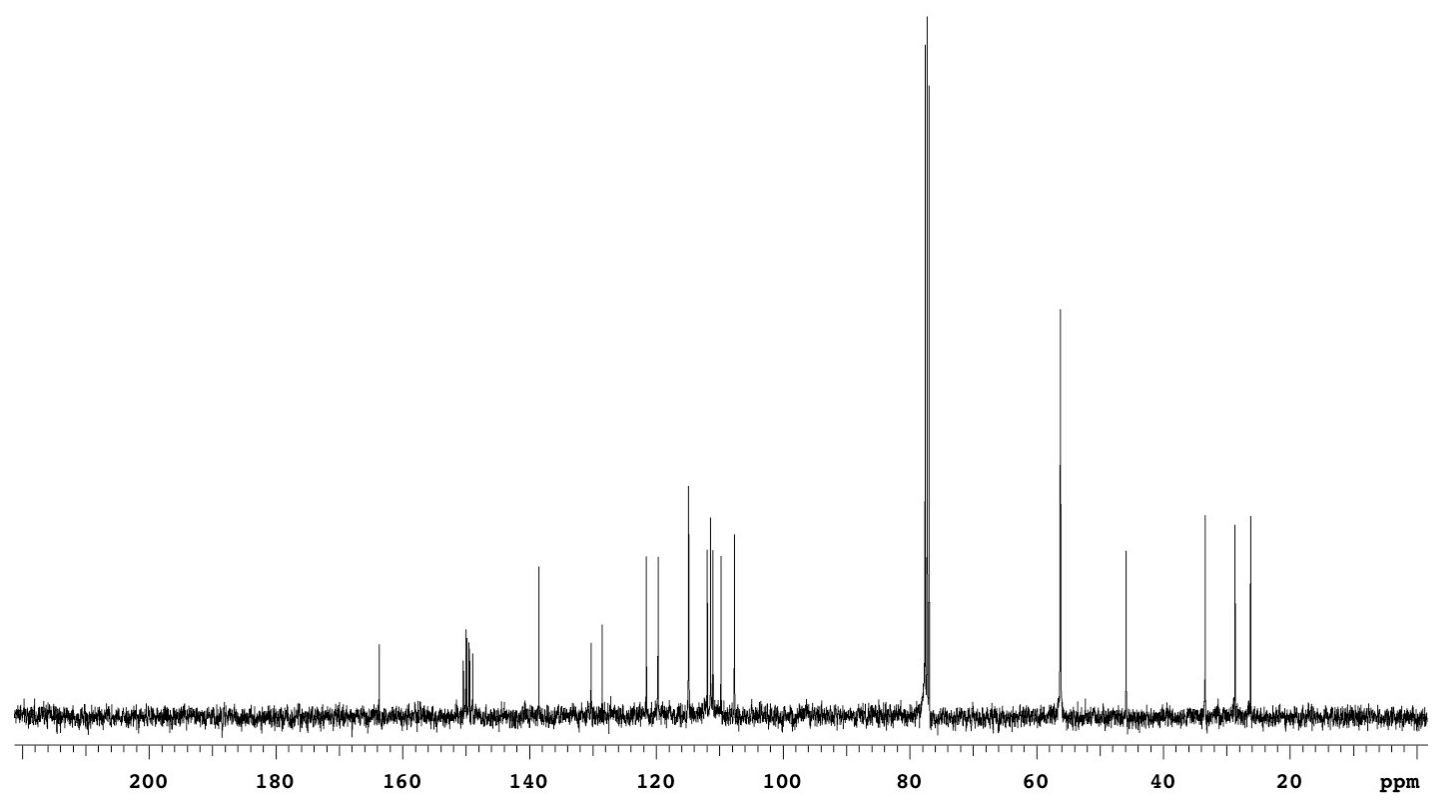




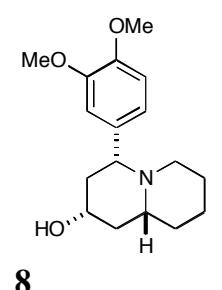

8

STANDARD 1H OBSERVE

yu2-301

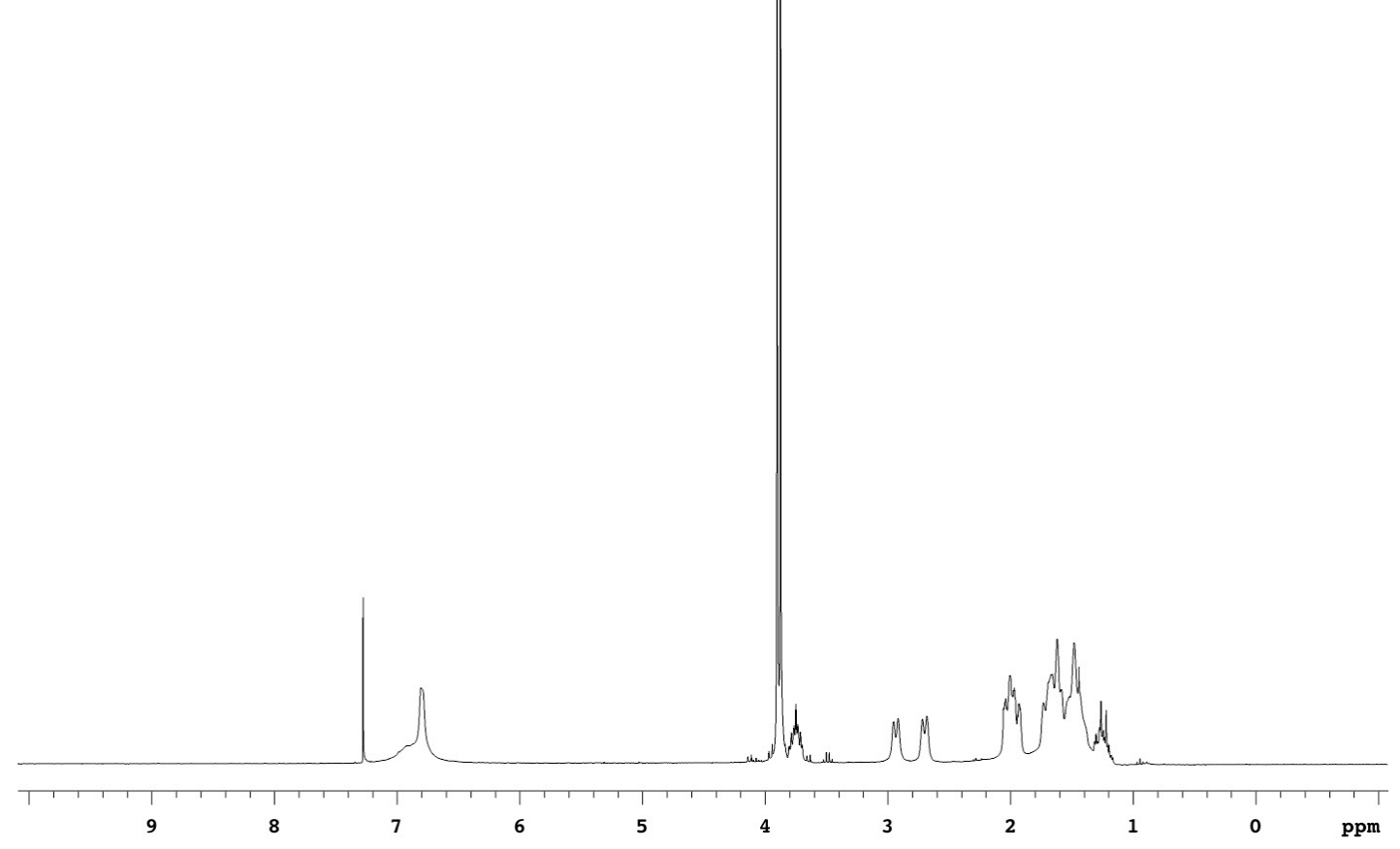

13C OBSERVE

yu2-301c
yu2-301

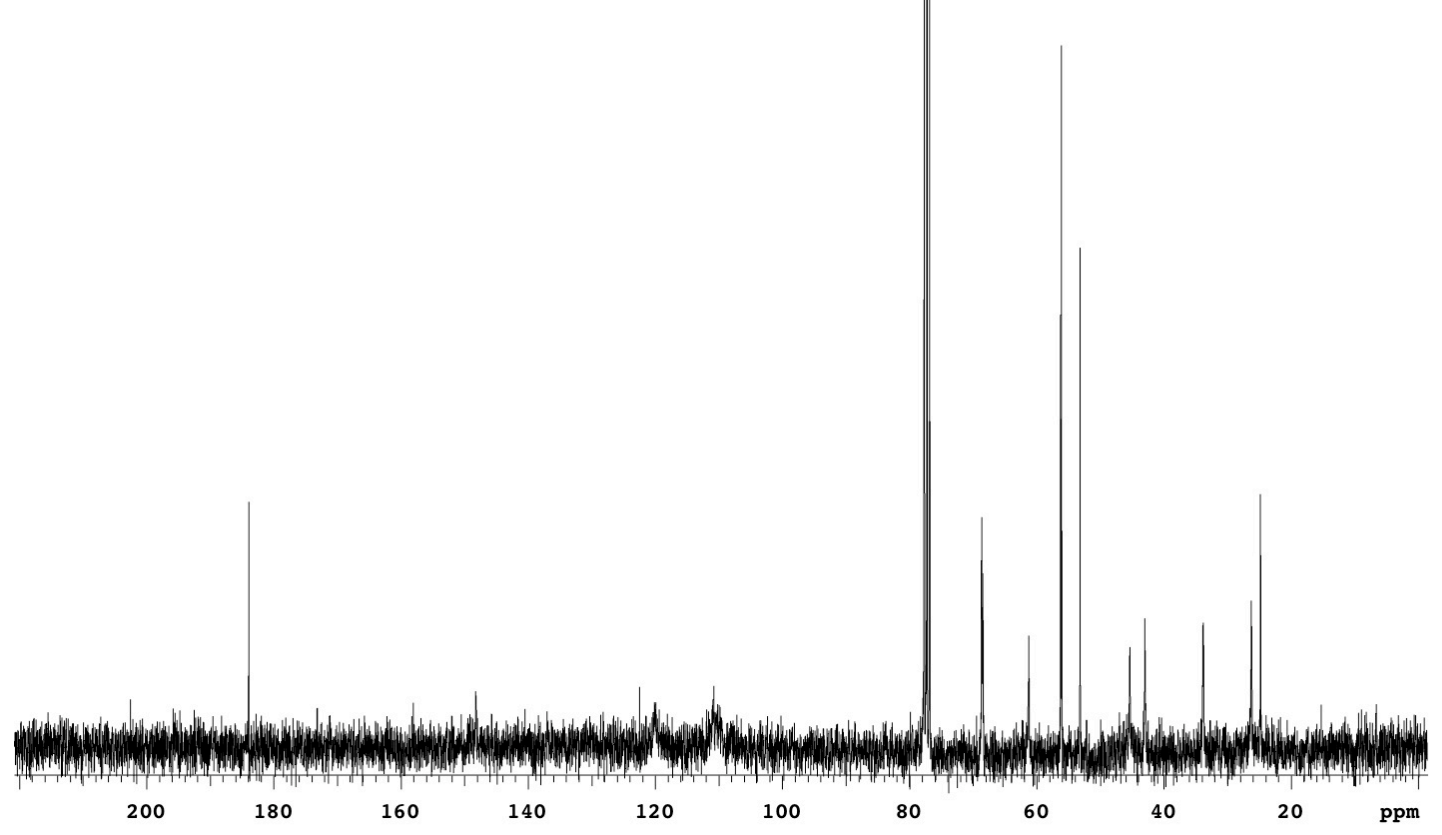




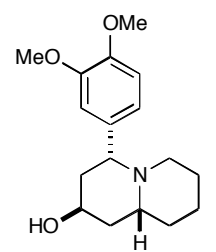

(+)-lasubine II

STANDRRD 1H OBSERVE

yu2-306-2

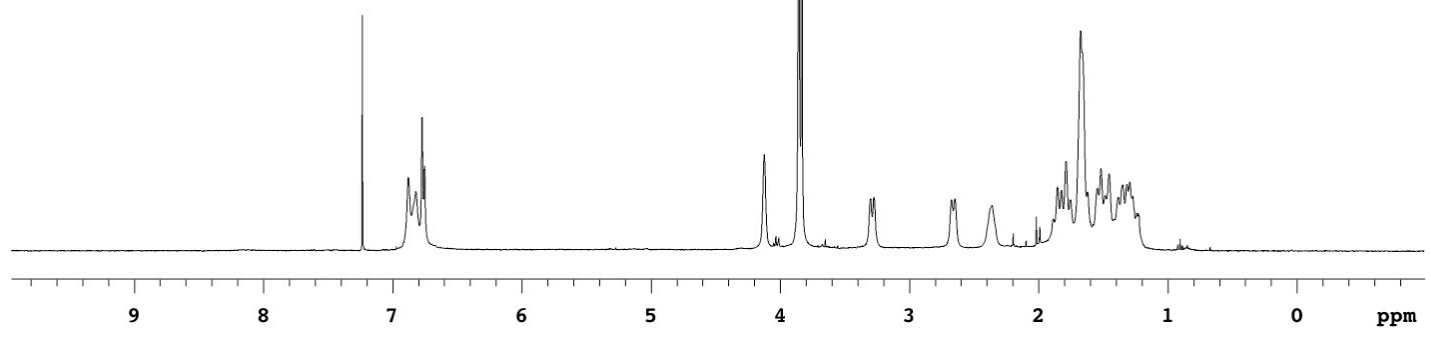

13C OBSERVE

yu2-306-2

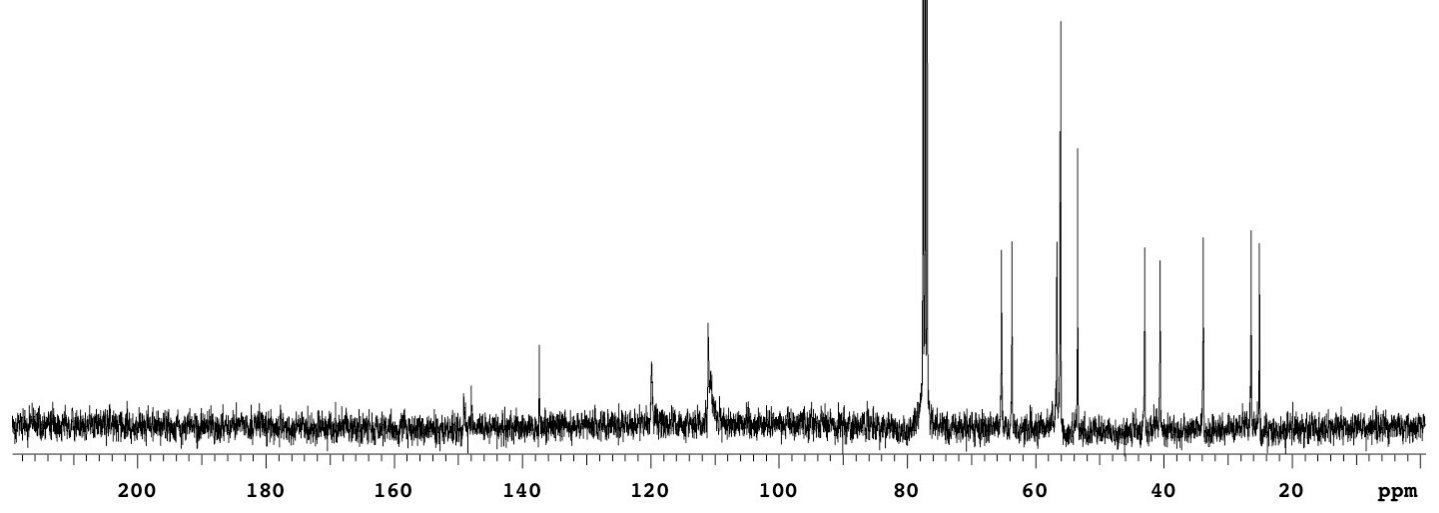


Table 1. Crystal data and structure refinement for $\mathbf{3 j}$.

Identification code

Empirical formula

Formula weight

Temperature

Wavelength

Crystal system

Space group

Unit cell dimensions

Volume

Z

Density (calculated)

Absorption coefficient

$\mathrm{F}(000)$

Crystal size

Theta range for data collection

Index ranges

Reflections collected

Independent reflections

Completeness to theta $=35.41^{\circ}$

Absorption correction

Max. and min. transmission

Refinement method

Data / restraints / parameters

Goodness-of-fit on $\mathrm{F}^{2}$

Final R indices [I $>2 \operatorname{sigma}(\mathrm{I})]$

$\mathrm{R}$ indices (all data)

Absolute structure parameter

Largest diff. peak and hole rovis $22 \mathrm{a}$

C14 H14 Br N O

292.17

373(2) K

$0.71073 \AA$

Monoclinic

P2(1)

$$
\begin{array}{ll}
\mathrm{a}=12.2073(5) \AA & \alpha=90^{\circ} . \\
\mathrm{b}=7.8184(4) \AA & \beta=91.258(3)^{\circ} . \\
\mathrm{c}=12.9408(6) \AA & \gamma=90^{\circ} .
\end{array}
$$

1234.79(10) $\AA^{3}$

4

$1.572 \mathrm{Mg} / \mathrm{m}^{3}$

$3.311 \mathrm{~mm}^{-1}$

592

$0.17 \times 0.15 \times 0.11 \mathrm{~mm}^{3}$

1.57 to $35.41^{\circ}$.

$-19<=\mathrm{h}<=19,-12<=\mathrm{k}<=12,-21<=1<=19$

50738

$10905[\mathrm{R}(\mathrm{int})=0.0466]$

$99.1 \%$

multi-scan

0.7183 and 0.6062

Full-matrix least-squares on $\mathrm{F}^{2}$

$10905 / 1 / 308$

0.940

$\mathrm{R} 1=0.0350, \mathrm{wR} 2=0.0719$

$\mathrm{R} 1=0.0722, \mathrm{wR} 2=0.0811$

$0.005(5)$

0.415 and -0.553 e. $\AA^{-3}$ 
Table 2. Atomic coordinates $\left(\times 10^{4}\right)$ and equivalent isotropic displacement parameters $\left(\AA^{2} \times 10^{3}\right)$ for $\mathbf{3 j}$. $U(e q)$ is defined as one third of the trace of the orthogonalized $\mathrm{U}^{\mathrm{ij}}$ tensor.

\begin{tabular}{|c|c|c|c|c|}
\hline & $\mathrm{x}$ & $\mathrm{y}$ & z & $\mathrm{U}(\mathrm{eq})$ \\
\hline $\operatorname{Br}(1 \mathrm{~A})$ & $11295(1)$ & $11742(1)$ & 7916(1) & $37(1)$ \\
\hline $\mathrm{N}(1 \mathrm{~A})$ & $17817(1)$ & $11759(3)$ & $11237(1)$ & $23(1)$ \\
\hline $\mathrm{O}(1 \mathrm{~A})$ & $16938(1)$ & 11933(3) & $12752(1)$ & $34(1)$ \\
\hline $\mathrm{C}(1 \mathrm{~A})$ & 17819(1) & 11803(3) & 10101(1) & $22(1)$ \\
\hline $\mathrm{C}(2 \mathrm{~A})$ & $18879(1)$ & $10840(3)$ & 9887(2) & $33(1)$ \\
\hline $\mathrm{C}(3 \mathrm{~A})$ & 19650(1) & $11386(4)$ & 10774(2) & $36(1)$ \\
\hline $\mathrm{C}(4 \mathrm{~A})$ & 18903(1) & 11572(3) & 11703(2) & $27(1)$ \\
\hline $\mathrm{C}(5 \mathrm{~A})$ & $16909(1)$ & 11934(3) & 11800(1) & $24(1)$ \\
\hline$C(6 A)$ & $15870(1)$ & $12096(2)$ & 11199(1) & $24(1)$ \\
\hline$C(7 A)$ & $15775(1)$ & 11685(3) & 10195(1) & $18(1)$ \\
\hline $\mathrm{C}(8 \mathrm{~A})$ & $16776(1)$ & $11087(3)$ & 9635(1) & $22(1)$ \\
\hline $\mathrm{C}(9 \mathrm{~A})$ & 14701(1) & 11718(3) & 9646(1) & $19(1)$ \\
\hline$C(10 A)$ & $13749(1)$ & $11235(2)$ & $10149(2)$ & $22(1)$ \\
\hline$C(11 \mathrm{~A})$ & $12736(1)$ & $11285(3)$ & 9643(2) & $26(1)$ \\
\hline$C(12 A)$ & $12675(1)$ & 11793(3) & $8623(2)$ & $24(1)$ \\
\hline$C(13 \mathrm{~A})$ & $13601(1)$ & $12305(3)$ & 8101(2) & $27(1)$ \\
\hline$C(14 \mathrm{~A})$ & $14611(1)$ & $12256(2)$ & $8618(2)$ & $23(1)$ \\
\hline $\operatorname{Br}(1)$ & $8590(1)$ & 9294(1) & 7116(1) & $34(1)$ \\
\hline $\mathrm{N}(1)$ & $2124(1)$ & $9645(2)$ & $3705(1)$ & $24(1)$ \\
\hline $\mathrm{O}(1)$ & $3018(1)$ & 9083(2) & $2230(1)$ & $31(1)$ \\
\hline$C(1)$ & $2117(1)$ & $10323(3)$ & 4764(1) & $24(1)$ \\
\hline$C(2)$ & 996(1) & 9774(3) & $5120(2)$ & $33(1)$ \\
\hline$C(3)$ & 271(1) & 9885(3) & 4142(2) & $31(1)$ \\
\hline$C(4)$ & $1027(1)$ & 9390(4) & $3257(2)$ & $29(1)$ \\
\hline$C(5)$ & $3044(1)$ & 9482(3) & 3159(1) & $24(1)$ \\
\hline$C(6)$ & 4082(1) & 9704(3) & 3743(1) & $24(1)$ \\
\hline$C(7)$ & 4145(1) & $9776(2)$ & 4780(1) & $20(1)$ \\
\hline $\mathrm{C}(8)$ & $3100(1)$ & $9726(3)$ & $5387(1)$ & $25(1)$ \\
\hline$C(9)$ & $5207(1)$ & $9747(2)$ & 5349(1) & $20(1)$ \\
\hline$C(10)$ & $6185(1)$ & $10219(3)$ & 4874(2) & $25(1)$ \\
\hline $\mathrm{C}(11)$ & 7181(1) & $10117(3)$ & $5396(2)$ & $27(1)$ \\
\hline
\end{tabular}


C(12)

C(13)

C(14)
7213(1)

6265(1)

5267(1)
9537(3)

9090(3)

9199(3)
6408(2)

6908(2)

6381(1)
25(1)

26(1)

24(1)

S-51 
Table 3. Bond lengths $[\AA]$ and angles $\left[^{\circ}\right]$ for $\mathbf{3 j}$.

\begin{tabular}{|c|c|}
\hline $\operatorname{Br}(1 \mathrm{~A})-\mathrm{C}(12 \mathrm{~A})$ & $1.9006(17)$ \\
\hline $\mathrm{N}(1 \mathrm{~A})-\mathrm{C}(5 \mathrm{~A})$ & $1.346(2)$ \\
\hline $\mathrm{N}(1 \mathrm{~A})-\mathrm{C}(4 \mathrm{~A})$ & $1.452(2)$ \\
\hline $\mathrm{N}(1 \mathrm{~A})-\mathrm{C}(1 \mathrm{~A})$ & $1.471(2)$ \\
\hline $\mathrm{O}(1 \mathrm{~A})-\mathrm{C}(5 \mathrm{~A})$ & $1.231(2)$ \\
\hline $\mathrm{C}(1 \mathrm{~A})-\mathrm{C}(8 \mathrm{~A})$ & $1.505(2)$ \\
\hline $\mathrm{C}(1 \mathrm{~A})-\mathrm{C}(2 \mathrm{~A})$ & $1.528(3)$ \\
\hline$C(2 A)-C(3 A)$ & $1.529(3)$ \\
\hline $\mathrm{C}(3 \mathrm{~A})-\mathrm{C}(4 \mathrm{~A})$ & $1.532(2)$ \\
\hline $\mathrm{C}(5 \mathrm{~A})-\mathrm{C}(6 \mathrm{~A})$ & $1.480(2)$ \\
\hline $\mathrm{C}(6 \mathrm{~A})-\mathrm{C}(7 \mathrm{~A})$ & $1.340(2)$ \\
\hline $\mathrm{C}(7 \mathrm{~A})-\mathrm{C}(9 \mathrm{~A})$ & $1.477(2)$ \\
\hline $\mathrm{C}(7 \mathrm{~A})-\mathrm{C}(8 \mathrm{~A})$ & $1.509(2)$ \\
\hline $\mathrm{C}(9 \mathrm{~A})-\mathrm{C}(10 \mathrm{~A})$ & $1.396(2)$ \\
\hline $\mathrm{C}(9 \mathrm{~A})-\mathrm{C}(14 \mathrm{~A})$ & $1.397(3)$ \\
\hline$C(10 \mathrm{~A})-\mathrm{C}(11 \mathrm{~A})$ & $1.387(2)$ \\
\hline$C(11 \mathrm{~A})-\mathrm{C}(12 \mathrm{~A})$ & $1.379(3)$ \\
\hline$C(12 A)-C(13 A)$ & $1.388(3)$ \\
\hline$C(13 A)-C(14 A)$ & $1.391(2)$ \\
\hline $\operatorname{Br}(1)-C(12)$ & $1.9053(17)$ \\
\hline $\mathrm{N}(1)-\mathrm{C}(5)$ & $1.347(2)$ \\
\hline $\mathrm{N}(1)-\mathrm{C}(4)$ & $1.461(2)$ \\
\hline $\mathrm{N}(1)-\mathrm{C}(1)$ & $1.468(2)$ \\
\hline $\mathrm{O}(1)-\mathrm{C}(5)$ & $1.242(2)$ \\
\hline $\mathrm{C}(1)-\mathrm{C}(8)$ & $1.506(2)$ \\
\hline$C(1)-C(2)$ & $1.515(2)$ \\
\hline $\mathrm{C}(2)-\mathrm{C}(3)$ & $1.532(3)$ \\
\hline$C(3)-C(4)$ & $1.536(3)$ \\
\hline$C(5)-C(6)$ & $1.471(2)$ \\
\hline$C(6)-C(7)$ & $1.344(3)$ \\
\hline $\mathrm{C}(7)-\mathrm{C}(9)$ & $1.477(2)$ \\
\hline $\mathrm{C}(7)-\mathrm{C}(8)$ & $1.512(2)$ \\
\hline$C(9)-C(14)$ & $1.403(3)$ \\
\hline$C(9)-C(10)$ & $1.404(2)$ \\
\hline
\end{tabular}




\begin{tabular}{|c|c|}
\hline$C(10)-C(11)$ & $1.380(3)$ \\
\hline $\mathrm{C}(11)-\mathrm{C}(12)$ & $1.386(3)$ \\
\hline$C(12)-C(13)$ & $1.383(2)$ \\
\hline$C(13)-C(14)$ & $1.385(2)$ \\
\hline $\mathrm{C}(5 \mathrm{~A})-\mathrm{N}(1 \mathrm{~A})-\mathrm{C}(4 \mathrm{~A})$ & $122.76(15)$ \\
\hline $\mathrm{C}(5 \mathrm{~A})-\mathrm{N}(1 \mathrm{~A})-\mathrm{C}(1 \mathrm{~A})$ & $123.94(14)$ \\
\hline $\mathrm{C}(4 \mathrm{~A})-\mathrm{N}(1 \mathrm{~A})-\mathrm{C}(1 \mathrm{~A})$ & $113.27(13)$ \\
\hline $\mathrm{N}(1 \mathrm{~A})-\mathrm{C}(1 \mathrm{~A})-\mathrm{C}(8 \mathrm{~A})$ & $111.81(14)$ \\
\hline $\mathrm{N}(1 \mathrm{~A})-\mathrm{C}(1 \mathrm{~A})-\mathrm{C}(2 \mathrm{~A})$ & $100.96(14)$ \\
\hline$C(8 \mathrm{~A})-\mathrm{C}(1 \mathrm{~A})-\mathrm{C}(2 \mathrm{~A})$ & $117.18(17)$ \\
\hline$C(1 \mathrm{~A})-\mathrm{C}(2 \mathrm{~A})-\mathrm{C}(3 \mathrm{~A})$ & $103.68(17)$ \\
\hline$C(2 A)-C(3 A)-C(4 A)$ & $104.38(14)$ \\
\hline $\mathrm{N}(1 \mathrm{~A})-\mathrm{C}(4 \mathrm{~A})-\mathrm{C}(3 \mathrm{~A})$ & $103.80(15)$ \\
\hline $\mathrm{O}(1 \mathrm{~A})-\mathrm{C}(5 \mathrm{~A})-\mathrm{N}(1 \mathrm{~A})$ & $122.35(16)$ \\
\hline $\mathrm{O}(1 \mathrm{~A})-\mathrm{C}(5 \mathrm{~A})-\mathrm{C}(6 \mathrm{~A})$ & $122.15(15)$ \\
\hline $\mathrm{N}(1 \mathrm{~A})-\mathrm{C}(5 \mathrm{~A})-\mathrm{C}(6 \mathrm{~A})$ & $115.50(16)$ \\
\hline$C(7 A)-C(6 A)-C(5 A)$ & $122.98(15)$ \\
\hline$C(6 A)-C(7 A)-C(9 A)$ & $121.29(15)$ \\
\hline$C(6 A)-C(7 A)-C(8 A)$ & $119.06(14)$ \\
\hline $\mathrm{C}(9 \mathrm{~A})-\mathrm{C}(7 \mathrm{~A})-\mathrm{C}(8 \mathrm{~A})$ & $119.57(15)$ \\
\hline $\mathrm{C}(1 \mathrm{~A})-\mathrm{C}(8 \mathrm{~A})-\mathrm{C}(7 \mathrm{~A})$ & $112.30(15)$ \\
\hline$C(10 A)-C(9 A)-C(14 A)$ & $118.43(15)$ \\
\hline$C(10 A)-C(9 A)-C(7 A)$ & $120.63(16)$ \\
\hline $\mathrm{C}(14 \mathrm{~A})-\mathrm{C}(9 \mathrm{~A})-\mathrm{C}(7 \mathrm{~A})$ & $120.94(15)$ \\
\hline$C(11 A)-C(10 A)-C(9 A)$ & $121.02(18)$ \\
\hline$C(12 A)-C(11 A)-C(10 A)$ & $119.19(16)$ \\
\hline$C(11 A)-C(12 A)-C(13 A)$ & $121.50(16)$ \\
\hline $\mathrm{C}(11 \mathrm{~A})-\mathrm{C}(12 \mathrm{~A})-\mathrm{Br}(1 \mathrm{~A})$ & $118.88(13)$ \\
\hline $\mathrm{C}(13 \mathrm{~A})-\mathrm{C}(12 \mathrm{~A})-\mathrm{Br}(1 \mathrm{~A})$ & $119.61(15)$ \\
\hline $\mathrm{C}(12 \mathrm{~A})-\mathrm{C}(13 \mathrm{~A})-\mathrm{C}(14 \mathrm{~A})$ & $118.71(19)$ \\
\hline $\mathrm{C}(13 \mathrm{~A})-\mathrm{C}(14 \mathrm{~A})-\mathrm{C}(9 \mathrm{~A})$ & $121.13(16)$ \\
\hline $\mathrm{C}(5)-\mathrm{N}(1)-\mathrm{C}(4)$ & $123.14(16)$ \\
\hline $\mathrm{C}(5)-\mathrm{N}(1)-\mathrm{C}(1)$ & $123.06(15)$ \\
\hline $\mathrm{C}(4)-\mathrm{N}(1)-\mathrm{C}(1)$ & $113.30(13)$ \\
\hline $\mathrm{N}(1)-\mathrm{C}(1)-\mathrm{C}(8)$ & $111.52(14)$ \\
\hline
\end{tabular}




\begin{tabular}{ll}
$\mathrm{N}(1)-\mathrm{C}(1)-\mathrm{C}(2)$ & $101.90(15)$ \\
$\mathrm{C}(8)-\mathrm{C}(1)-\mathrm{C}(2)$ & $117.63(16)$ \\
$\mathrm{C}(1)-\mathrm{C}(2)-\mathrm{C}(3)$ & $103.98(15)$ \\
$\mathrm{C}(2)-\mathrm{C}(3)-\mathrm{C}(4)$ & $104.83(14)$ \\
$\mathrm{N}(1)-\mathrm{C}(4)-\mathrm{C}(3)$ & $103.30(15)$ \\
$\mathrm{O}(1)-\mathrm{C}(5)-\mathrm{N}(1)$ & $121.82(16)$ \\
$\mathrm{O}(1)-\mathrm{C}(5)-\mathrm{C}(6)$ & $122.08(15)$ \\
$\mathrm{N}(1)-\mathrm{C}(5)-\mathrm{C}(6)$ & $116.01(16)$ \\
$\mathrm{C}(7)-\mathrm{C}(6)-\mathrm{C}(5)$ & $123.19(15)$ \\
$\mathrm{C}(6)-\mathrm{C}(7)-\mathrm{C}(9)$ & $121.79(15)$ \\
$\mathrm{C}(6)-\mathrm{C}(7)-\mathrm{C}(8)$ & $119.16(15)$ \\
$\mathrm{C}(9)-\mathrm{C}(7)-\mathrm{C}(8)$ & $118.82(15)$ \\
$\mathrm{C}(1)-\mathrm{C}(8)-\mathrm{C}(7)$ & $112.67(15)$ \\
$\mathrm{C}(14)-\mathrm{C}(9)-\mathrm{C}(10)$ & $118.05(15)$ \\
$\mathrm{C}(14)-\mathrm{C}(9)-\mathrm{C}(7)$ & $120.36(15)$ \\
$\mathrm{C}(10)-\mathrm{C}(9)-\mathrm{C}(7)$ & $121.57(17)$ \\
$\mathrm{C}(11)-\mathrm{C}(10)-\mathrm{C}(9)$ & $121.26(19)$ \\
$\mathrm{C}(10)-\mathrm{C}(11)-\mathrm{C}(12)$ & $119.21(16)$ \\
$\mathrm{C}(13)-\mathrm{C}(12)-\mathrm{C}(11)$ & $121.17(16)$ \\
$\mathrm{C}(13)-\mathrm{C}(12)-\mathrm{Br}(1)$ & $119.26(15)$ \\
$\mathrm{C}(11)-\mathrm{C}(12)-\mathrm{Br}(1)$ & $119.55(13)$ \\
$\mathrm{C}(12)-\mathrm{C}(13)-\mathrm{C}(14)$ & $119.38(18)$ \\
$\mathrm{C}(13)-\mathrm{C}(14)-\mathrm{C}(9)$ & $120.91(15)$ \\
& \\
\hline
\end{tabular}

Symmetry transformations used to generate equivalent atoms: 
Table 4. Anisotropic displacement parameters $\left(\AA^{2} \times 10^{3}\right)$ for $\mathbf{3 j}$. The anisotropic displacement factor exponent takes the form: $-2 \pi^{2}\left[h^{2} a^{* 2} U^{11}+\ldots+2 h k a^{*} b^{*} U^{12}\right]$

\begin{tabular}{|c|c|c|c|c|c|c|}
\hline & $\mathrm{U}^{11}$ & $\mathrm{U}^{22}$ & $\mathrm{U}^{33}$ & $\mathrm{U}^{23}$ & $\mathrm{U}^{13}$ & $\mathrm{U}^{12}$ \\
\hline $\operatorname{Br}(1 \mathrm{~A})$ & 19(1) & $48(1)$ & $45(1)$ & $-8(1)$ & $-6(1)$ & $1(1)$ \\
\hline $\mathrm{N}(1 \mathrm{~A})$ & 19(1) & $35(1)$ & $15(1)$ & $-1(1)$ & $2(1)$ & $2(1)$ \\
\hline $\mathrm{O}(1 \mathrm{~A})$ & $30(1)$ & $59(1)$ & $14(1)$ & $-4(1)$ & $4(1)$ & $5(1)$ \\
\hline$C(1 \mathrm{~A})$ & $21(1)$ & $28(1)$ & $16(1)$ & $3(1)$ & $4(1)$ & $-2(1)$ \\
\hline$C(2 A)$ & $19(1)$ & $58(1)$ & $22(1)$ & $-3(1)$ & $6(1)$ & $3(1)$ \\
\hline$C(3 \mathrm{~A})$ & $18(1)$ & $62(2)$ & $28(1)$ & $-1(1)$ & $3(1)$ & $-5(1)$ \\
\hline$C(4 A)$ & $20(1)$ & $37(1)$ & $24(1)$ & $0(1)$ & $-1(1)$ & $0(1)$ \\
\hline$C(5 \mathrm{~A})$ & $22(1)$ & $34(1)$ & $18(1)$ & $-1(1)$ & $4(1)$ & $2(1)$ \\
\hline$C(6 A)$ & $21(1)$ & $31(1)$ & $20(1)$ & $0(1)$ & $6(1)$ & $2(1)$ \\
\hline $\mathrm{C}(7 \mathrm{~A})$ & $18(1)$ & $20(1)$ & $17(1)$ & $3(1)$ & $4(1)$ & $-1(1)$ \\
\hline $\mathrm{C}(8 \mathrm{~A})$ & $19(1)$ & $29(1)$ & $18(1)$ & $-1(1)$ & $3(1)$ & $4(1)$ \\
\hline $\mathrm{C}(9 \mathrm{~A})$ & $18(1)$ & $20(1)$ & $20(1)$ & $-1(1)$ & $4(1)$ & $0(1)$ \\
\hline$C(10 A)$ & $21(1)$ & $23(1)$ & $23(1)$ & $2(1)$ & $6(1)$ & $1(1)$ \\
\hline$C(11 \mathrm{~A})$ & $17(1)$ & $22(1)$ & $40(1)$ & $-3(1)$ & $8(1)$ & $0(1)$ \\
\hline $\mathrm{C}(12 \mathrm{~A})$ & $17(1)$ & $25(1)$ & $31(1)$ & $-5(1)$ & $-1(1)$ & $0(1)$ \\
\hline$C(13 A)$ & $22(1)$ & $35(1)$ & $22(1)$ & $1(1)$ & $-1(1)$ & $-1(1)$ \\
\hline $\mathrm{C}(14 \mathrm{~A})$ & $20(1)$ & $29(1)$ & $21(1)$ & $1(1)$ & $2(1)$ & $-2(1)$ \\
\hline $\operatorname{Br}(1)$ & $18(1)$ & $47(1)$ & $37(1)$ & $-8(1)$ & $-6(1)$ & $0(1)$ \\
\hline $\mathrm{N}(1)$ & $18(1)$ & $36(1)$ & $16(1)$ & $-3(1)$ & $0(1)$ & $4(1)$ \\
\hline $\mathrm{O}(1)$ & $27(1)$ & $50(1)$ & $17(1)$ & $-2(1)$ & $1(1)$ & $7(1)$ \\
\hline $\mathrm{C}(1)$ & $20(1)$ & $34(1)$ & $18(1)$ & $-2(1)$ & $4(1)$ & $3(1)$ \\
\hline$C(2)$ & $20(1)$ & $56(2)$ & $22(1)$ & $-1(1)$ & $4(1)$ & $1(1)$ \\
\hline$C(3)$ & $18(1)$ & $47(1)$ & $29(1)$ & $-5(1)$ & $2(1)$ & $5(1)$ \\
\hline$C(4)$ & $18(1)$ & $43(1)$ & $26(1)$ & $-8(1)$ & $-3(1)$ & $7(1)$ \\
\hline$C(5)$ & $20(1)$ & $31(1)$ & 19(1) & $2(1)$ & $3(1)$ & $5(1)$ \\
\hline$C(6)$ & $18(1)$ & $37(1)$ & $18(1)$ & $1(1)$ & $5(1)$ & $1(1)$ \\
\hline$C(7)$ & $18(1)$ & $22(1)$ & $20(1)$ & $2(1)$ & $4(1)$ & $0(1)$ \\
\hline$C(8)$ & $18(1)$ & $41(1)$ & $16(1)$ & $-3(1)$ & $4(1)$ & $0(1)$ \\
\hline$C(9)$ & $17(1)$ & $23(1)$ & $21(1)$ & $-2(1)$ & $3(1)$ & $0(1)$ \\
\hline$C(10)$ & $20(1)$ & $32(1)$ & $23(1)$ & $3(1)$ & $6(1)$ & $-1(1)$ \\
\hline $\mathrm{C}(11)$ & $18(1)$ & $33(1)$ & $30(1)$ & $1(1)$ & $6(1)$ & $-2(1)$ \\
\hline
\end{tabular}




\begin{tabular}{lllllll}
$\mathrm{C}(12)$ & $15(1)$ & $26(1)$ & $32(1)$ & $-6(1)$ & $-2(1)$ & $-1(1)$ \\
$\mathrm{C}(13)$ & $20(1)$ & $35(1)$ & $23(1)$ & $1(1)$ & $-2(1)$ & $-4(1)$ \\
$\mathrm{C}(14)$ & $17(1)$ & $35(1)$ & $19(1)$ & $2(1)$ & $1(1)$ & $-4(1)$ \\
\hline
\end{tabular}


Table 5. Hydrogen coordinates ( $\left.\times 10^{4}\right)$ and isotropic displacement parameters $\left(\AA^{2} \times 10^{3}\right)$ for $\mathbf{3 j}$.

\begin{tabular}{|c|c|c|c|c|}
\hline & $\mathrm{x}$ & $\mathrm{y}$ & z & $\mathrm{U}(\mathrm{eq})$ \\
\hline $\mathrm{H}(1 \mathrm{AA})$ & 17899 & 12992 & 9875 & 26 \\
\hline $\mathrm{H}(2 \mathrm{AA})$ & 19170 & 11168 & 9224 & 39 \\
\hline $\mathrm{H}(2 \mathrm{AB})$ & 18761 & 9614 & 9893 & 39 \\
\hline $\mathrm{H}(3 \mathrm{AA})$ & 20005 & 12463 & 10618 & 43 \\
\hline $\mathrm{H}(3 \mathrm{AB})$ & 20208 & 10525 & 10905 & 43 \\
\hline $\mathrm{H}(4 \mathrm{AA})$ & 18944 & 10566 & 12141 & 32 \\
\hline $\mathrm{H}(4 \mathrm{AB})$ & 19101 & 12570 & 12111 & 32 \\
\hline $\mathrm{H}(6 \mathrm{AA})$ & 15254 & 12501 & 11532 & 29 \\
\hline $\mathrm{H}(8 \mathrm{AA})$ & 16808 & 9847 & 9656 & 26 \\
\hline $\mathrm{H}(8 \mathrm{AB})$ & 16715 & 11432 & 8916 & 26 \\
\hline $\mathrm{H}(10 \mathrm{~A})$ & 13795 & 10873 & 10833 & 27 \\
\hline $\mathrm{H}(11 \mathrm{~A})$ & 12104 & 10981 & 9988 & 31 \\
\hline $\mathrm{H}(13 \mathrm{~A})$ & 13546 & 12674 & 7419 & 32 \\
\hline $\mathrm{H}(14 \mathrm{~A})$ & 15237 & 12588 & 8274 & 28 \\
\hline $\mathrm{H}(1 \mathrm{~A})$ & 2133 & 11574 & 4729 & 29 \\
\hline $\mathrm{H}(2 \mathrm{~A})$ & 733 & 10536 & 5652 & 39 \\
\hline $\mathrm{H}(2 \mathrm{~B})$ & 1016 & 8616 & 5389 & 39 \\
\hline $\mathrm{H}(3 \mathrm{~A})$ & -11 & 11035 & 4046 & 37 \\
\hline $\mathrm{H}(3 \mathrm{~B})$ & -342 & 9098 & 4180 & 37 \\
\hline $\mathrm{H}(4 \mathrm{~A})$ & 916 & 8208 & 3053 & 35 \\
\hline $\mathrm{H}(4 \mathrm{~B})$ & 904 & 10121 & 2660 & 35 \\
\hline $\mathrm{H}(6 \mathrm{~A})$ & 4726 & 9799 & 3377 & 29 \\
\hline $\mathrm{H}(8 \mathrm{~A})$ & 2975 & 8564 & 5620 & 30 \\
\hline $\mathrm{H}(8 \mathrm{~B})$ & 3188 & 10444 & 5994 & 30 \\
\hline $\mathrm{H}(10 \mathrm{~B})$ & 6162 & 10608 & 4195 & 30 \\
\hline $\mathrm{H}(11 \mathrm{~B})$ & 7823 & 10433 & 5072 & 32 \\
\hline $\mathrm{H}(13 \mathrm{~B})$ & 6297 & 8719 & 7590 & 31 \\
\hline $\mathrm{H}(14 \mathrm{~B})$ & 4628 & 8905 & 6716 & 29 \\
\hline
\end{tabular}




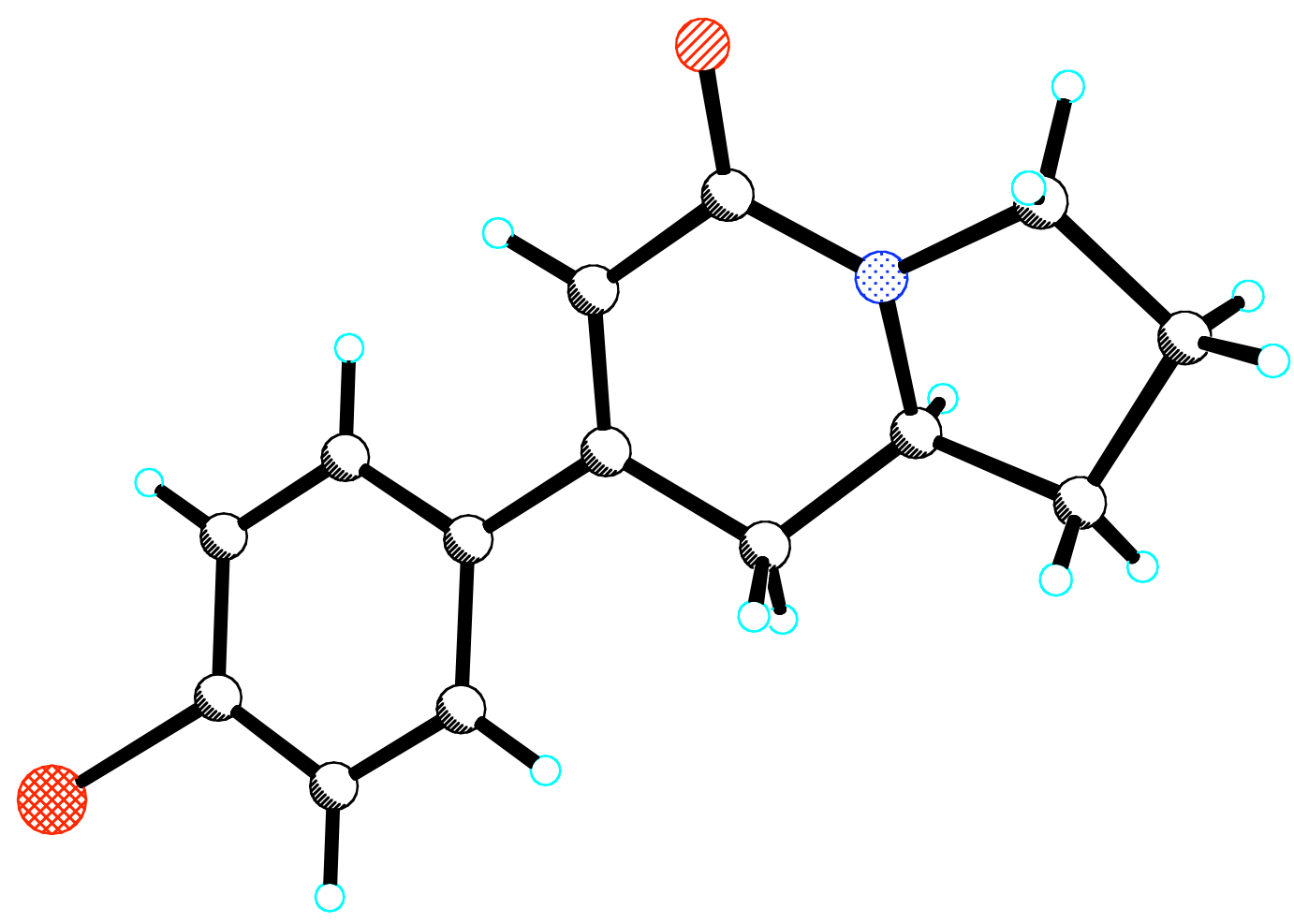

Figure 1 Br1 


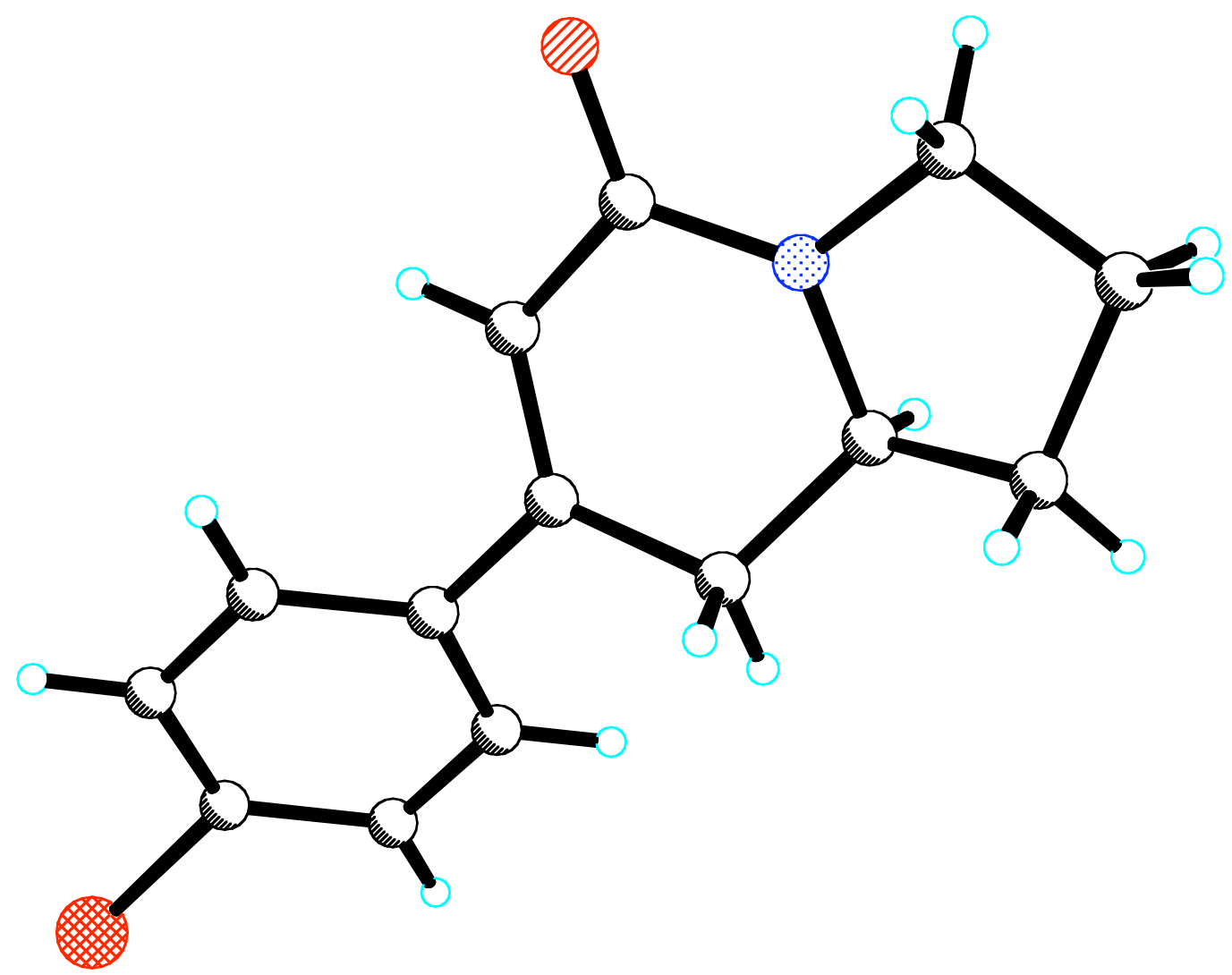

Figure 2 br1a 
Table 1. Crystal data and structure refinement for $\mathbf{4 j}$.

Identification code

Empirical formula

Formula weight

Temperature

Wavelength

Crystal system

Space group

Unit cell dimensions

Volume

Z

Density (calculated)

Absorption coefficient

$\mathrm{F}(000)$

Crystal size

Theta range for data collection

Index ranges

Reflections collected

Independent reflections

Completeness to theta $=31.53^{\circ}$

Absorption correction

Max. and min. transmission

Refinement method

Data / restraints / parameters

Goodness-of-fit on $\mathrm{F}^{2}$

Final $R$ indices [I $>2 \operatorname{sigma}(\mathrm{I})]$

$\mathrm{R}$ indices (all data)

Absolute structure parameter

Largest diff. peak and hole rovis $23 \mathrm{a}$

C14 H14 Br N O

292.17

$373(2) \mathrm{K}$

$0.71073 \AA$

Monoclinic

P2(1)

$$
\begin{array}{ll}
\mathrm{a}=12.5864(8) \AA & \alpha=90^{\circ} . \\
\mathrm{b}=8.6454(6) \AA & \beta=100.668(4)^{\circ} . \\
\mathrm{c}=17.4451(12) \AA & \gamma=90^{\circ} .
\end{array}
$$

$1865.5(2) \AA^{3}$

6

$1.560 \mathrm{Mg} / \mathrm{m}^{3}$

$3.288 \mathrm{~mm}^{-1}$

888

$0.13 \times 0.09 \times 0.09 \mathrm{~mm}^{3}$

1.65 to $31.53^{\circ}$.

$-18<=\mathrm{h}<=18,-12<=\mathrm{k}<=12,-25<=\mathrm{l}<=25$

55388

$12412[\mathrm{R}(\mathrm{int})=0.0498]$

$99.7 \%$

multi-scan

0.7541 and 0.6783

Full-matrix least-squares on $\mathrm{F}^{2}$

12412 / 1 / 461

0.744

$\mathrm{R} 1=0.0327, \mathrm{wR} 2=0.0806$

$\mathrm{R} 1=0.0482, \mathrm{wR} 2=0.0895$

$-0.006(5)$

0.583 and -0.511 e. $\AA^{-3}$ 
Table 2. Atomic coordinates $\left(\times 10^{4}\right)$ and equivalent isotropic displacement parameters $\left(\AA^{2} \times 10^{3}\right)$ for $\mathbf{4 j}$. $U(e q)$ is defined as one third of the trace of the orthogonalized $U^{i j}$ tensor.

\begin{tabular}{|c|c|c|c|c|}
\hline & $\mathrm{x}$ & $\mathrm{y}$ & $\mathrm{z}$ & $\mathrm{U}(\mathrm{eq})$ \\
\hline $\operatorname{Br}(1)$ & 1341(1) & $7216(1)$ & $6210(1)$ & $28(1)$ \\
\hline $\mathrm{N}(1)$ & $-2477(2)$ & $7614(2)$ & 2761(1) & $17(1)$ \\
\hline $\mathrm{O}(1)$ & $-992(2)$ & $9676(2)$ & $1119(1)$ & $20(1)$ \\
\hline$C(1)$ & $-1439(2)$ & $8026(3)$ & 2933(1) & $16(1)$ \\
\hline$C(2)$ & $-933(2)$ & $8835(3)$ & 2412(1) & $18(1)$ \\
\hline$C(3)$ & $-1423(2)$ & $9005(3)$ & 1610(1) & $17(1)$ \\
\hline$C(4)$ & $-2506(2)$ & $8183(3)$ & 1364(1) & $19(1)$ \\
\hline$C(5)$ & $-3156(2)$ & $8132(3)$ & 2016(1) & $18(1)$ \\
\hline$C(6)$ & $-4096(2)$ & $6989(3)$ & 1899(1) & $20(1)$ \\
\hline$C(7)$ & $-4206(2)$ & $6535(3)$ & $2730(2)$ & $21(1)$ \\
\hline $\mathrm{C}(8)$ & $-3043(2)$ & $6476(3)$ & 3173(1) & $20(1)$ \\
\hline$C(9)$ & $-808(2)$ & 7681(3) & 3729(1) & $17(1)$ \\
\hline$C(10)$ & $-1182(2)$ & $8187(3)$ & 4394(1) & $21(1)$ \\
\hline$C(11)$ & $-539(2)$ & $8024(3)$ & 5129(1) & $22(1)$ \\
\hline$C(12)$ & $463(2)$ & $7337(3)$ & 5196(1) & $20(1)$ \\
\hline$C(13)$ & $849(2)$ & $6772(3)$ & 4552(1) & $20(1)$ \\
\hline$C(14)$ & $192(2)$ & $6954(3)$ & 3819(1) & $18(1)$ \\
\hline $\operatorname{Br}(1 \mathrm{~A})$ & 2976(1) & $5554(1)$ & $13079(1)$ & $20(1)$ \\
\hline $\mathrm{N}(1 \mathrm{~A})$ & $238(2)$ & $7837(2)$ & 9512(1) & $14(1)$ \\
\hline $\mathrm{O}(1 \mathrm{~A})$ & $2189(2)$ & $9322(2)$ & $8017(1)$ & $28(1)$ \\
\hline$C(1 \mathrm{~A})$ & $1309(2)$ & 7987(3) & 9751(1) & $15(1)$ \\
\hline $\mathrm{C}(2 \mathrm{~A})$ & 1974(2) & $8625(3)$ & 9283(1) & $20(1)$ \\
\hline$C(3 \mathrm{~A})$ & $1605(2)$ & $8835(3)$ & 8463(1) & $21(1)$ \\
\hline$C(4 A)$ & $460(2)$ & $8293(3)$ & 8144(1) & $20(1)$ \\
\hline$C(5 A)$ & $-288(2)$ & $8421(3)$ & $8735(1)$ & $17(1)$ \\
\hline$C(6 A)$ & $-1326(2)$ & $7471(3)$ & 8553(1) & $21(1)$ \\
\hline$C(7 A)$ & $-1598(2)$ & $7136(3)$ & 9353(1) & $21(1)$ \\
\hline $\mathrm{C}(8 \mathrm{~A})$ & $-488(2)$ & $6830(3)$ & 9863(1) & $17(1)$ \\
\hline $\mathrm{C}(9 \mathrm{~A})$ & 1764(2) & $7455(3)$ & $10557(1)$ & $15(1)$ \\
\hline$C(10 A)$ & 1341(2) & $8031(3)$ & $11190(1)$ & $17(1)$ \\
\hline $\mathrm{C}(11 \mathrm{~A})$ & 1734(2) & 7483(3) & 11940(1) & $17(1)$ \\
\hline
\end{tabular}




\begin{tabular}{lrrrr}
$\mathrm{C}(12 \mathrm{~A})$ & $2546(2)$ & $6368(3)$ & $12059(1)$ & $17(1)$ \\
$\mathrm{C}(13 \mathrm{~A})$ & $2998(2)$ & $5823(3)$ & $11437(1)$ & $18(1)$ \\
$\mathrm{C}(14 \mathrm{~A})$ & $2599(2)$ & $6373(3)$ & $10687(1)$ & $17(1)$ \\
$\mathrm{Br}(1 \mathrm{~B})$ & $5095(1)$ & $7231(1)$ & $9810(1)$ & $41(1)$ \\
$\mathrm{N}(1 \mathrm{~B})$ & $6128(2)$ & $5254(2)$ & $6176(1)$ & $19(1)$ \\
$\mathrm{O}(1 \mathrm{~B})$ & $3274(2)$ & $4358(3)$ & $4680(1)$ & $30(1)$ \\
$\mathrm{C}(1 \mathrm{~B})$ & $5172(2)$ & $5403(3)$ & $6412(1)$ & $18(1)$ \\
$\mathrm{C}(2 \mathrm{~B})$ & $4204(2)$ & $5029(3)$ & $5935(1)$ & $23(1)$ \\
$\mathrm{C}(3 \mathrm{~B})$ & $4132(2)$ & $4687(3)$ & $5123(1)$ & $22(1)$ \\
$\mathrm{C}(4 \mathrm{~B})$ & $5192(2)$ & $4883(4)$ & $4817(2)$ & $29(1)$ \\
$\mathrm{C}(5 \mathrm{~B})$ & $6177(2)$ & $4495(4)$ & $5430(2)$ & $27(1)$ \\
$\mathrm{C}(6 \mathrm{~B})$ & $7266(2)$ & $4964(4)$ & $5261(2)$ & $29(1)$ \\
$\mathrm{C}(7 \mathrm{~B})$ & $7955(2)$ & $5175(4)$ & $6078(2)$ & $31(1)$ \\
$\mathrm{C}(8 \mathrm{~B})$ & $7174(2)$ & $5950(3)$ & $6527(2)$ & $24(1)$ \\
$\mathrm{C}(9 \mathrm{~B})$ & $5165(2)$ & $5896(3)$ & $7230(1)$ & $18(1)$ \\
$\mathrm{C}(10 \mathrm{~B})$ & $5825(2)$ & $5115(3)$ & $7847(1)$ & $20(1)$ \\
$\mathrm{C}(11 \mathrm{~B})$ & $5780(2)$ & $5502(4)$ & $8614(1)$ & $24(1)$ \\
$\mathrm{C}(12 \mathrm{~B})$ & $5103(2)$ & $6672(3)$ & $8757(2)$ & $24(1)$ \\
$\mathrm{C}(13 \mathrm{~B})$ & $4435(2)$ & $7461(3)$ & $8163(2)$ & $23(1)$ \\
$\mathrm{C}(14 \mathrm{~B})$ & $4473(2)$ & $7053(3)$ & $7392(1)$ & $22(1)$ \\
& & & & \\
\hline
\end{tabular}


Table 3. Bond lengths $[\AA]$ and angles $\left[^{\circ}\right]$ for $\mathbf{4 j}$.

\begin{tabular}{|c|c|}
\hline $\operatorname{Br}(1)-C(12)$ & $1.906(2)$ \\
\hline $\mathrm{N}(1)-\mathrm{C}(1)$ & $1.334(3)$ \\
\hline $\mathrm{N}(1)-\mathrm{C}(8)$ & $1.477(3)$ \\
\hline $\mathrm{N}(1)-\mathrm{C}(5)$ & $1.487(3)$ \\
\hline $\mathrm{O}(1)-\mathrm{C}(3)$ & $1.240(3)$ \\
\hline $\mathrm{C}(1)-\mathrm{C}(2)$ & $1.391(3)$ \\
\hline$C(1)-C(9)$ & $1.496(3)$ \\
\hline $\mathrm{C}(2)-\mathrm{C}(3)$ & $1.429(3)$ \\
\hline$C(3)-C(4)$ & $1.527(3)$ \\
\hline $\mathrm{C}(4)-\mathrm{C}(5)$ & $1.519(3)$ \\
\hline$C(5)-C(6)$ & $1.526(3)$ \\
\hline$C(6)-C(7)$ & $1.532(3)$ \\
\hline$C(7)-C(8)$ & $1.525(4)$ \\
\hline$C(9)-C(14)$ & $1.389(3)$ \\
\hline$C(9)-C(10)$ & $1.400(3)$ \\
\hline$C(10)-C(11)$ & $1.391(3)$ \\
\hline$C(11)-C(12)$ & $1.380(4)$ \\
\hline$C(12)-C(13)$ & $1.393(3)$ \\
\hline$C(13)-C(14)$ & $1.397(3)$ \\
\hline $\mathrm{Br}(1 \mathrm{~A})-\mathrm{C}(12 \mathrm{~A})$ & $1.896(2)$ \\
\hline $\mathrm{N}(1 \mathrm{~A})-\mathrm{C}(1 \mathrm{~A})$ & $1.342(3)$ \\
\hline $\mathrm{N}(1 \mathrm{~A})-\mathrm{C}(8 \mathrm{~A})$ & $1.475(3)$ \\
\hline$N(1 \mathrm{~A})-\mathrm{C}(5 \mathrm{~A})$ & $1.482(3)$ \\
\hline $\mathrm{O}(1 \mathrm{~A})-\mathrm{C}(3 \mathrm{~A})$ & $1.239(3)$ \\
\hline$C(1 \mathrm{~A})-\mathrm{C}(2 \mathrm{~A})$ & $1.387(3)$ \\
\hline$C(1 \mathrm{~A})-\mathrm{C}(9 \mathrm{~A})$ & $1.490(3)$ \\
\hline$C(2 A)-C(3 A)$ & $1.431(3)$ \\
\hline$C(3 A)-C(4 A)$ & $1.520(4)$ \\
\hline$C(4 A)-C(5 A)$ & $1.523(3)$ \\
\hline$C(5 A)-C(6 A)$ & $1.526(3)$ \\
\hline$C(6 A)-C(7 A)$ & $1.526(3)$ \\
\hline$C(7 A)-C(8 A)$ & $1.533(3)$ \\
\hline $\mathrm{C}(9 \mathrm{~A})-\mathrm{C}(14 \mathrm{~A})$ & $1.394(3)$ \\
\hline$C(9 A)-C(10 A)$ & $1.403(3)$ \\
\hline
\end{tabular}




\begin{tabular}{|c|c|}
\hline$C(10 A)-C(11 A)$ & $1.394(3)$ \\
\hline$C(11 \mathrm{~A})-\mathrm{C}(12 \mathrm{~A})$ & $1.392(3)$ \\
\hline$C(12 A)-C(13 A)$ & $1.398(3)$ \\
\hline$C(13 A)-C(14 A)$ & $1.396(3)$ \\
\hline $\mathrm{Br}(1 \mathrm{~B})-\mathrm{C}(12 \mathrm{~B})$ & $1.902(3)$ \\
\hline $\mathrm{N}(1 \mathrm{~B})-\mathrm{C}(1 \mathrm{~B})$ & $1.348(3)$ \\
\hline $\mathrm{N}(1 \mathrm{~B})-\mathrm{C}(5 \mathrm{~B})$ & $1.469(3)$ \\
\hline $\mathrm{N}(1 \mathrm{~B})-\mathrm{C}(8 \mathrm{~B})$ & $1.474(3)$ \\
\hline $\mathrm{O}(1 \mathrm{~B})-\mathrm{C}(3 \mathrm{~B})$ & $1.239(3)$ \\
\hline$C(1 B)-C(2 B)$ & $1.381(3)$ \\
\hline$C(1 B)-C(9 B)$ & $1.490(3)$ \\
\hline$C(2 B)-C(3 B)$ & $1.433(3)$ \\
\hline$C(3 B)-C(4 B)$ & $1.535(4)$ \\
\hline $\mathrm{C}(4 \mathrm{~B})-\mathrm{C}(5 \mathrm{~B})$ & $1.517(4)$ \\
\hline $\mathrm{C}(5 \mathrm{~B})-\mathrm{C}(6 \mathrm{~B})$ & $1.510(4)$ \\
\hline$C(6 B)-C(7 B)$ & $1.536(4)$ \\
\hline $\mathrm{C}(7 \mathrm{~B})-\mathrm{C}(8 \mathrm{~B})$ & $1.521(4)$ \\
\hline $\mathrm{C}(9 \mathrm{~B})-\mathrm{C}(14 \mathrm{~B})$ & $1.390(3)$ \\
\hline $\mathrm{C}(9 \mathrm{~B})-\mathrm{C}(10 \mathrm{~B})$ & $1.405(3)$ \\
\hline$C(10 B)-C(11 B)$ & $1.389(3)$ \\
\hline $\mathrm{C}(11 \mathrm{~B})-\mathrm{C}(12 \mathrm{~B})$ & $1.375(4)$ \\
\hline$C(12 B)-C(13 B)$ & $1.386(4)$ \\
\hline$C(13 B)-C(14 B)$ & $1.400(3)$ \\
\hline $\mathrm{C}(1)-\mathrm{N}(1)-\mathrm{C}(8)$ & $127.5(2)$ \\
\hline $\mathrm{C}(1)-\mathrm{N}(1)-\mathrm{C}(5)$ & 119.77(19) \\
\hline $\mathrm{C}(8)-\mathrm{N}(1)-\mathrm{C}(5)$ & $112.06(19)$ \\
\hline $\mathrm{N}(1)-\mathrm{C}(1)-\mathrm{C}(2)$ & $122.3(2)$ \\
\hline $\mathrm{N}(1)-\mathrm{C}(1)-\mathrm{C}(9)$ & $119.0(2)$ \\
\hline $\mathrm{C}(2)-\mathrm{C}(1)-\mathrm{C}(9)$ & $118.6(2)$ \\
\hline $\mathrm{C}(1)-\mathrm{C}(2)-\mathrm{C}(3)$ & $121.7(2)$ \\
\hline $\mathrm{O}(1)-\mathrm{C}(3)-\mathrm{C}(2)$ & $124.0(2)$ \\
\hline $\mathrm{O}(1)-\mathrm{C}(3)-\mathrm{C}(4)$ & $120.4(2)$ \\
\hline$C(2)-C(3)-C(4)$ & $115.3(2)$ \\
\hline $\mathrm{C}(5)-\mathrm{C}(4)-\mathrm{C}(3)$ & $112.31(19)$ \\
\hline $\mathrm{N}(1)-\mathrm{C}(5)-\mathrm{C}(4)$ & $111.21(19)$ \\
\hline
\end{tabular}




\begin{tabular}{|c|c|}
\hline$N(1)-C(5)-C(6)$ & $103.15(19)$ \\
\hline$C(4)-C(5)-C(6)$ & $115.5(2)$ \\
\hline$C(5)-C(6)-C(7)$ & $104.08(19)$ \\
\hline$C(8)-C(7)-C(6)$ & $104.01(19)$ \\
\hline $\mathrm{N}(1)-\mathrm{C}(8)-\mathrm{C}(7)$ & $103.60(19)$ \\
\hline$C(14)-C(9)-C(10)$ & $119.0(2)$ \\
\hline$C(14)-C(9)-C(1)$ & $120.6(2)$ \\
\hline $\mathrm{C}(10)-\mathrm{C}(9)-\mathrm{C}(1)$ & $120.3(2)$ \\
\hline$C(11)-C(10)-C(9)$ & $120.3(2)$ \\
\hline $\mathrm{C}(12)-\mathrm{C}(11)-\mathrm{C}(10)$ & $119.2(2)$ \\
\hline$C(11)-C(12)-C(13)$ & $122.2(2)$ \\
\hline$C(11)-C(12)-B r(1)$ & $117.81(18)$ \\
\hline$C(13)-C(12)-B r(1)$ & $120.01(19)$ \\
\hline $\mathrm{C}(12)-\mathrm{C}(13)-\mathrm{C}(14)$ & $117.6(2)$ \\
\hline $\mathrm{C}(9)-\mathrm{C}(14)-\mathrm{C}(13)$ & $121.7(2)$ \\
\hline $\mathrm{C}(1 \mathrm{~A})-\mathrm{N}(1 \mathrm{~A})-\mathrm{C}(8 \mathrm{~A})$ & $126.32(19)$ \\
\hline $\mathrm{C}(1 \mathrm{~A})-\mathrm{N}(1 \mathrm{~A})-\mathrm{C}(5 \mathrm{~A})$ & $120.21(19)$ \\
\hline $\mathrm{C}(8 \mathrm{~A})-\mathrm{N}(1 \mathrm{~A})-\mathrm{C}(5 \mathrm{~A})$ & $112.03(18)$ \\
\hline $\mathrm{N}(1 \mathrm{~A})-\mathrm{C}(1 \mathrm{~A})-\mathrm{C}(2 \mathrm{~A})$ & $122.5(2)$ \\
\hline $\mathrm{N}(1 \mathrm{~A})-\mathrm{C}(1 \mathrm{~A})-\mathrm{C}(9 \mathrm{~A})$ & $116.8(2)$ \\
\hline $\mathrm{C}(2 \mathrm{~A})-\mathrm{C}(1 \mathrm{~A})-\mathrm{C}(9 \mathrm{~A})$ & $120.6(2)$ \\
\hline $\mathrm{C}(1 \mathrm{~A})-\mathrm{C}(2 \mathrm{~A})-\mathrm{C}(3 \mathrm{~A})$ & $121.3(2)$ \\
\hline $\mathrm{O}(1 \mathrm{~A})-\mathrm{C}(3 \mathrm{~A})-\mathrm{C}(2 \mathrm{~A})$ & $123.4(2)$ \\
\hline $\mathrm{O}(1 \mathrm{~A})-\mathrm{C}(3 \mathrm{~A})-\mathrm{C}(4 \mathrm{~A})$ & $120.5(2)$ \\
\hline $\mathrm{C}(2 \mathrm{~A})-\mathrm{C}(3 \mathrm{~A})-\mathrm{C}(4 \mathrm{~A})$ & $115.9(2)$ \\
\hline$C(3 A)-C(4 A)-C(5 A)$ & $113.1(2)$ \\
\hline $\mathrm{N}(1 \mathrm{~A})-\mathrm{C}(5 \mathrm{~A})-\mathrm{C}(4 \mathrm{~A})$ & $111.56(19)$ \\
\hline $\mathrm{N}(1 \mathrm{~A})-\mathrm{C}(5 \mathrm{~A})-\mathrm{C}(6 \mathrm{~A})$ & $103.12(18)$ \\
\hline $\mathrm{C}(4 \mathrm{~A})-\mathrm{C}(5 \mathrm{~A})-\mathrm{C}(6 \mathrm{~A})$ & $115.6(2)$ \\
\hline$C(7 A)-C(6 A)-C(5 A)$ & $103.86(19)$ \\
\hline $\mathrm{C}(6 \mathrm{~A})-\mathrm{C}(7 \mathrm{~A})-\mathrm{C}(8 \mathrm{~A})$ & $103.26(19)$ \\
\hline $\mathrm{N}(1 \mathrm{~A})-\mathrm{C}(8 \mathrm{~A})-\mathrm{C}(7 \mathrm{~A})$ & $102.99(18)$ \\
\hline $\mathrm{C}(14 \mathrm{~A})-\mathrm{C}(9 \mathrm{~A})-\mathrm{C}(10 \mathrm{~A})$ & $119.7(2)$ \\
\hline $\mathrm{C}(14 \mathrm{~A})-\mathrm{C}(9 \mathrm{~A})-\mathrm{C}(1 \mathrm{~A})$ & $120.4(2)$ \\
\hline$C(10 A)-C(9 A)-C(1 A)$ & $119.9(2)$ \\
\hline$C(11 A)-C(10 A)-C(9 A)$ & $119.9(2)$ \\
\hline
\end{tabular}




\begin{tabular}{|c|c|}
\hline$C(12 A)-C(11 A)-C(10 A)$ & $119.9(2)$ \\
\hline$C(11 A)-C(12 A)-C(13 A)$ & $120.7(2)$ \\
\hline$C(11 \mathrm{~A})-\mathrm{C}(12 \mathrm{~A})-\mathrm{Br}(1 \mathrm{~A})$ & $118.11(18)$ \\
\hline$C(13 \mathrm{~A})-\mathrm{C}(12 \mathrm{~A})-\mathrm{Br}(1 \mathrm{~A})$ & $121.12(18)$ \\
\hline$C(14 A)-C(13 A)-C(12 A)$ & $119.1(2)$ \\
\hline$C(9 A)-C(14 A)-C(13 A)$ & $120.7(2)$ \\
\hline$C(1 B)-N(1 B)-C(5 B)$ & $120.0(2)$ \\
\hline$C(1 B)-N(1 B)-C(8 B)$ & $127.8(2)$ \\
\hline $\mathrm{C}(5 \mathrm{~B})-\mathrm{N}(1 \mathrm{~B})-\mathrm{C}(8 \mathrm{~B})$ & $111.7(2)$ \\
\hline $\mathrm{N}(1 \mathrm{~B})-\mathrm{C}(1 \mathrm{~B})-\mathrm{C}(2 \mathrm{~B})$ & $122.1(2)$ \\
\hline $\mathrm{N}(1 \mathrm{~B})-\mathrm{C}(1 \mathrm{~B})-\mathrm{C}(9 \mathrm{~B})$ & $118.9(2)$ \\
\hline$C(2 B)-C(1 B)-C(9 B)$ & $118.9(2)$ \\
\hline$C(1 B)-C(2 B)-C(3 B)$ & $122.1(2)$ \\
\hline $\mathrm{O}(1 \mathrm{~B})-\mathrm{C}(3 \mathrm{~B})-\mathrm{C}(2 \mathrm{~B})$ & $123.5(2)$ \\
\hline $\mathrm{O}(1 \mathrm{~B})-\mathrm{C}(3 \mathrm{~B})-\mathrm{C}(4 \mathrm{~B})$ & $121.4(2)$ \\
\hline$C(2 B)-C(3 B)-C(4 B)$ & $114.9(2)$ \\
\hline $\mathrm{C}(5 \mathrm{~B})-\mathrm{C}(4 \mathrm{~B})-\mathrm{C}(3 \mathrm{~B})$ & $112.4(2)$ \\
\hline $\mathrm{N}(1 \mathrm{~B})-\mathrm{C}(5 \mathrm{~B})-\mathrm{C}(6 \mathrm{~B})$ & $104.0(2)$ \\
\hline N(1B)-C(5B)-C(4B) & $111.3(2)$ \\
\hline $\mathrm{C}(6 \mathrm{~B})-\mathrm{C}(5 \mathrm{~B})-\mathrm{C}(4 \mathrm{~B})$ & $117.2(2)$ \\
\hline $\mathrm{C}(5 \mathrm{~B})-\mathrm{C}(6 \mathrm{~B})-\mathrm{C}(7 \mathrm{~B})$ & $103.2(2)$ \\
\hline$C(8 B)-C(7 B)-C(6 B)$ & $102.8(2)$ \\
\hline N(1B)-C(8B)-C(7B) & $102.7(2)$ \\
\hline $\mathrm{C}(14 \mathrm{~B})-\mathrm{C}(9 \mathrm{~B})-\mathrm{C}(10 \mathrm{~B})$ & $119.5(2)$ \\
\hline $\mathrm{C}(14 \mathrm{~B})-\mathrm{C}(9 \mathrm{~B})-\mathrm{C}(1 \mathrm{~B})$ & $121.1(2)$ \\
\hline $\mathrm{C}(10 \mathrm{~B})-\mathrm{C}(9 \mathrm{~B})-\mathrm{C}(1 \mathrm{~B})$ & $119.3(2)$ \\
\hline C(11B)-C(10B)-C(9B) & $119.9(2)$ \\
\hline$C(12 B)-C(11 B)-C(10 B)$ & $119.2(2)$ \\
\hline$C(11 B)-C(12 B)-C(13 B)$ & $122.5(2)$ \\
\hline$C(11 B)-C(12 B)-B r(1 B)$ & $118.4(2)$ \\
\hline$C(13 B)-C(12 B)-B r(1 B)$ & $119.1(2)$ \\
\hline $\mathrm{C}(12 \mathrm{~B})-\mathrm{C}(13 \mathrm{~B})-\mathrm{C}(14 \mathrm{~B})$ & $118.0(2)$ \\
\hline $\mathrm{C}(9 \mathrm{~B})-\mathrm{C}(14 \mathrm{~B})-\mathrm{C}(13 \mathrm{~B})$ & $120.8(2)$ \\
\hline
\end{tabular}

Symmetry transformations used to generate equivalent atoms: 
S-67 
Table 4. Anisotropic displacement parameters $\left(\AA^{2} \times 10^{3}\right)$ for $4 \mathbf{j}$. The anisotropic displacement factor exponent takes the form: $-2 \pi^{2}\left[h^{2} a^{* 2} U^{11}+\ldots+2 h k a^{*} b^{*} U^{12}\right]$

\begin{tabular}{|c|c|c|c|c|c|c|}
\hline & $\mathrm{U}^{11}$ & $\mathrm{U}^{22}$ & $\mathrm{U}^{33}$ & $\mathrm{U}^{23}$ & $\mathrm{U}^{13}$ & $\mathrm{U}^{12}$ \\
\hline $\operatorname{Br}(1)$ & $33(1)$ & $33(1)$ & $14(1)$ & $3(1)$ & $-3(1)$ & $-2(1)$ \\
\hline $\mathrm{N}(1)$ & $20(1)$ & $19(1)$ & $12(1)$ & $2(1)$ & $4(1)$ & $1(1)$ \\
\hline $\mathrm{O}(1)$ & $25(1)$ & $18(1)$ & $18(1)$ & $4(1)$ & $7(1)$ & $1(1)$ \\
\hline $\mathrm{C}(1)$ & $19(1)$ & $16(1)$ & $12(1)$ & $-3(1)$ & $2(1)$ & $2(1)$ \\
\hline$C(2)$ & $20(1)$ & $18(1)$ & $16(1)$ & $-1(1)$ & $5(1)$ & $-3(1)$ \\
\hline$C(3)$ & $18(1)$ & $16(1)$ & $17(1)$ & $1(1)$ & $6(1)$ & $3(1)$ \\
\hline$C(4)$ & $21(1)$ & $24(1)$ & $12(1)$ & $2(1)$ & $2(1)$ & $-1(1)$ \\
\hline$C(5)$ & $20(1)$ & $20(1)$ & $15(1)$ & $3(1)$ & $2(1)$ & $2(1)$ \\
\hline$C(6)$ & $16(1)$ & $25(1)$ & $18(1)$ & $1(1)$ & $4(1)$ & $-1(1)$ \\
\hline$C(7)$ & $22(1)$ & $21(1)$ & $21(1)$ & $0(1)$ & $8(1)$ & $-2(1)$ \\
\hline$C(8)$ & $21(1)$ & $24(1)$ & $17(1)$ & $4(1)$ & $8(1)$ & $-1(1)$ \\
\hline$C(9)$ & $20(1)$ & $17(1)$ & $14(1)$ & $1(1)$ & $2(1)$ & $-2(1)$ \\
\hline$C(10)$ & $23(1)$ & $22(1)$ & $17(1)$ & $-1(1)$ & $3(1)$ & $3(1)$ \\
\hline$C(11)$ & $31(1)$ & $21(1)$ & $15(1)$ & $-1(1)$ & $6(1)$ & $-1(1)$ \\
\hline$C(12)$ & $26(1)$ & $19(1)$ & $12(1)$ & $2(1)$ & $0(1)$ & $-2(1)$ \\
\hline$C(13)$ & $21(1)$ & $20(1)$ & $18(1)$ & $3(1)$ & $2(1)$ & $1(1)$ \\
\hline$C(14)$ & $24(1)$ & $15(1)$ & $14(1)$ & $1(1)$ & $4(1)$ & $0(1)$ \\
\hline $\operatorname{Br}(1 \mathrm{~A})$ & $21(1)$ & $25(1)$ & $12(1)$ & $3(1)$ & $-1(1)$ & $-1(1)$ \\
\hline $\mathrm{N}(1 \mathrm{~A})$ & $16(1)$ & $14(1)$ & 11(1) & $1(1)$ & $1(1)$ & $0(1)$ \\
\hline $\mathrm{O}(1 \mathrm{~A})$ & $31(1)$ & $34(1)$ & $21(1)$ & $7(1)$ & $9(1)$ & $-5(1)$ \\
\hline$C(1 \mathrm{~A})$ & $18(1)$ & $12(1)$ & $13(1)$ & $-2(1)$ & $1(1)$ & $0(1)$ \\
\hline $\mathrm{C}(2 \mathrm{~A})$ & $18(1)$ & $23(1)$ & $16(1)$ & $2(1)$ & $2(1)$ & $-6(1)$ \\
\hline$C(3 \mathrm{~A})$ & $25(1)$ & $18(1)$ & $20(1)$ & $3(1)$ & $6(1)$ & $-2(1)$ \\
\hline$C(4 A)$ & $26(1)$ & $22(1)$ & $12(1)$ & $3(1)$ & $2(1)$ & $0(1)$ \\
\hline$C(5 \mathrm{~A})$ & $18(1)$ & $16(1)$ & $14(1)$ & $2(1)$ & $-1(1)$ & $0(1)$ \\
\hline$C(6 A)$ & $19(1)$ & $21(1)$ & $19(1)$ & $0(1)$ & $-4(1)$ & $0(1)$ \\
\hline$C(7 A)$ & $15(1)$ & $22(1)$ & $25(1)$ & $1(1)$ & $2(1)$ & $2(1)$ \\
\hline $\mathrm{C}(8 \mathrm{~A})$ & $20(1)$ & $14(1)$ & $17(1)$ & $1(1)$ & $2(1)$ & $-2(1)$ \\
\hline $\mathrm{C}(9 \mathrm{~A})$ & $17(1)$ & $15(1)$ & $13(1)$ & $1(1)$ & $2(1)$ & $-2(1)$ \\
\hline$C(10 A)$ & $18(1)$ & $16(1)$ & $16(1)$ & $-1(1)$ & $0(1)$ & $-1(1)$ \\
\hline$C(11 \mathrm{~A})$ & $20(1)$ & $20(1)$ & $13(1)$ & $-1(1)$ & $3(1)$ & $-3(1)$ \\
\hline
\end{tabular}




\begin{tabular}{lllllll}
$\mathrm{C}(12 \mathrm{~A})$ & $19(1)$ & $19(1)$ & $13(1)$ & $2(1)$ & $0(1)$ & $-3(1)$ \\
$\mathrm{C}(13 \mathrm{~A})$ & $17(1)$ & $20(1)$ & $17(1)$ & $1(1)$ & $1(1)$ & $-1(1)$ \\
$\mathrm{C}(14 \mathrm{~A})$ & $18(1)$ & $21(1)$ & $13(1)$ & $1(1)$ & $5(1)$ & $-1(1)$ \\
$\mathrm{Br}(1 \mathrm{~B})$ & $32(1)$ & $72(1)$ & $20(1)$ & $-16(1)$ & $7(1)$ & $-9(1)$ \\
$\mathrm{N}(1 \mathrm{~B})$ & $19(1)$ & $22(1)$ & $17(1)$ & $-3(1)$ & $4(1)$ & $0(1)$ \\
$\mathrm{O}(1 \mathrm{~B})$ & $28(1)$ & $44(1)$ & $16(1)$ & $2(1)$ & $-1(1)$ & $-6(1)$ \\
$\mathrm{C}(1 \mathrm{~B})$ & $19(1)$ & $18(1)$ & $17(1)$ & $0(1)$ & $5(1)$ & $1(1)$ \\
$\mathrm{C}(2 \mathrm{~B})$ & $19(1)$ & $32(1)$ & $16(1)$ & $-3(1)$ & $3(1)$ & $-4(1)$ \\
$\mathrm{C}(3 \mathrm{~B})$ & $25(1)$ & $25(1)$ & $15(1)$ & $5(1)$ & $2(1)$ & $0(1)$ \\
$\mathrm{C}(4 \mathrm{~B})$ & $28(2)$ & $43(2)$ & $17(1)$ & $-1(1)$ & $4(1)$ & $3(1)$ \\
$\mathrm{C}(5 \mathrm{~B})$ & $28(1)$ & $37(2)$ & $17(1)$ & $-4(1)$ & $7(1)$ & $5(1)$ \\
$\mathrm{C}(6 \mathrm{~B})$ & $24(1)$ & $47(2)$ & $18(1)$ & $7(1)$ & $8(1)$ & $11(1)$ \\
$\mathrm{C}(7 \mathrm{~B})$ & $24(1)$ & $45(2)$ & $26(1)$ & $4(1)$ & $8(1)$ & $3(1)$ \\
$\mathrm{C}(8 \mathrm{~B})$ & $18(1)$ & $28(1)$ & $27(1)$ & $1(1)$ & $5(1)$ & $-1(1)$ \\
$\mathrm{C}(9 \mathrm{~B})$ & $17(1)$ & $19(1)$ & $18(1)$ & $-2(1)$ & $4(1)$ & $-2(1)$ \\
$\mathrm{C}(10 \mathrm{~B})$ & $16(1)$ & $23(1)$ & $20(1)$ & $0(1)$ & $2(1)$ & $-1(1)$ \\
$\mathrm{C}(11 \mathrm{~B})$ & $20(1)$ & $33(1)$ & $16(1)$ & $5(1)$ & $-1(1)$ & $-1(1)$ \\
$\mathrm{C}(12 \mathrm{~B})$ & $22(1)$ & $34(1)$ & $18(1)$ & $-7(1)$ & $4(1)$ & $-11(1)$ \\
$\mathrm{C}(13 \mathrm{~B})$ & $24(1)$ & $21(1)$ & $25(1)$ & $-6(1)$ & $8(1)$ & $-2(1)$ \\
$\mathrm{C}(14 \mathrm{~B})$ & $22(1)$ & $21(1)$ & $22(1)$ & $-1(1)$ & $4(1)$ & $1(1)$ \\
& & & & & & \\
\hline
\end{tabular}


Table 5. Hydrogen coordinates ( $\left.\times 10^{4}\right)$ and isotropic displacement parameters $\left(\AA^{2} \times 10^{3}\right)$ for $\mathbf{4 j}$.

\begin{tabular}{|c|c|c|c|c|}
\hline & $\mathrm{x}$ & $\mathrm{y}$ & $\mathrm{z}$ & $\mathrm{U}(\mathrm{eq})$ \\
\hline $\mathrm{H}(2 \mathrm{~A})$ & -259 & 9277 & 2590 & 21 \\
\hline $\mathrm{H}(4 \mathrm{~A})$ & -2924 & 8713 & 918 & 23 \\
\hline $\mathrm{H}(4 \mathrm{~B})$ & -2378 & 7134 & 1205 & 23 \\
\hline $\mathrm{H}(5 \mathrm{~A})$ & -3434 & 9170 & 2088 & 22 \\
\hline $\mathrm{H}(6 \mathrm{~A})$ & -3937 & 6092 & 1606 & 24 \\
\hline $\mathrm{H}(6 \mathrm{~B})$ & -4754 & 7471 & 1626 & 24 \\
\hline $\mathrm{H}(7 \mathrm{~A})$ & -4622 & 7299 & 2955 & 25 \\
\hline $\mathrm{H}(7 \mathrm{~B})$ & -4554 & 5534 & 2736 & 25 \\
\hline $\mathrm{H}(8 \mathrm{~A})$ & -3001 & 6764 & 3715 & 24 \\
\hline $\mathrm{H}(8 \mathrm{~B})$ & -2738 & 5450 & 3150 & 24 \\
\hline $\mathrm{H}(10 \mathrm{~A})$ & -1864 & 8634 & 4344 & 25 \\
\hline $\mathrm{H}(11 \mathrm{~A})$ & -782 & 8375 & 5570 & 26 \\
\hline $\mathrm{H}(13 \mathrm{~A})$ & 1520 & 6291 & 4607 & 24 \\
\hline $\mathrm{H}(14 \mathrm{~A})$ & 429 & 6578 & 3381 & 21 \\
\hline $\mathrm{H}(2 \mathrm{AA})$ & 2672 & 8923 & 9507 & 23 \\
\hline $\mathrm{H}(4 \mathrm{AA})$ & 482 & 7224 & 7979 & 24 \\
\hline $\mathrm{H}(4 \mathrm{AB})$ & 165 & 8905 & 7688 & 24 \\
\hline $\mathrm{H}(5 \mathrm{AA})$ & -478 & 9511 & 8785 & 20 \\
\hline H(6AA) & -1900 & 8054 & 8232 & 25 \\
\hline $\mathrm{H}(6 \mathrm{AB})$ & -1211 & 6519 & 8285 & 25 \\
\hline H(7AA) & -2063 & 6238 & 9336 & 25 \\
\hline $\mathrm{H}(7 \mathrm{AB})$ & -1949 & 8016 & 9545 & 25 \\
\hline $\mathrm{H}(8 \mathrm{AA})$ & -483 & 7110 & 10402 & 20 \\
\hline $\mathrm{H}(8 \mathrm{AB})$ & -284 & 5751 & 9839 & 20 \\
\hline $\mathrm{H}(10 \mathrm{C})$ & 799 & 8777 & 11109 & 20 \\
\hline $\mathrm{H}(11 \mathrm{C})$ & 1455 & 7862 & 12361 & 21 \\
\hline $\mathrm{H}(13 \mathrm{C})$ & 3557 & 5104 & 11522 & 22 \\
\hline $\mathrm{H}(14 \mathrm{C})$ & 2893 & 6014 & 10269 & 20 \\
\hline $\mathrm{H}(2 \mathrm{BA})$ & 3578 & 4999 & 6147 & 27 \\
\hline $\mathrm{H}(4 \mathrm{BA})$ & 5178 & 4215 & 4368 & 35 \\
\hline
\end{tabular}




\begin{tabular}{lllll}
$\mathrm{H}(4 \mathrm{BB})$ & 5248 & 5943 & 4647 & 35 \\
$\mathrm{H}(5 \mathrm{BA})$ & 6186 & 3374 & 5514 & 33 \\
$\mathrm{H}(6 \mathrm{BA})$ & 7216 & 5921 & 4966 & 35 \\
$\mathrm{H}(6 \mathrm{BB})$ & 7563 & 4164 & 4973 & 35 \\
$\mathrm{H}(7 \mathrm{BA})$ & 8201 & 4187 & 6308 & 37 \\
$\mathrm{H}(7 \mathrm{BB})$ & 8577 & 5828 & 6061 & 37 \\
$\mathrm{H}(8 \mathrm{BA})$ & 7360 & 5721 & 7080 & 29 \\
$\mathrm{H}(8 \mathrm{BB})$ & 7165 & 7062 & 6454 & 29 \\
$\mathrm{H}(10 \mathrm{~B})$ & 6292 & 4342 & 7743 & 24 \\
$\mathrm{H}(11 \mathrm{~B})$ & 6203 & 4975 & 9025 & 28 \\
$\mathrm{H}(13 \mathrm{~B})$ & 3974 & 8237 & 8274 & 27 \\
$\mathrm{H}(14 \mathrm{~B})$ & 4031 & 7564 & 6984 & 26 \\
\hline
\end{tabular}
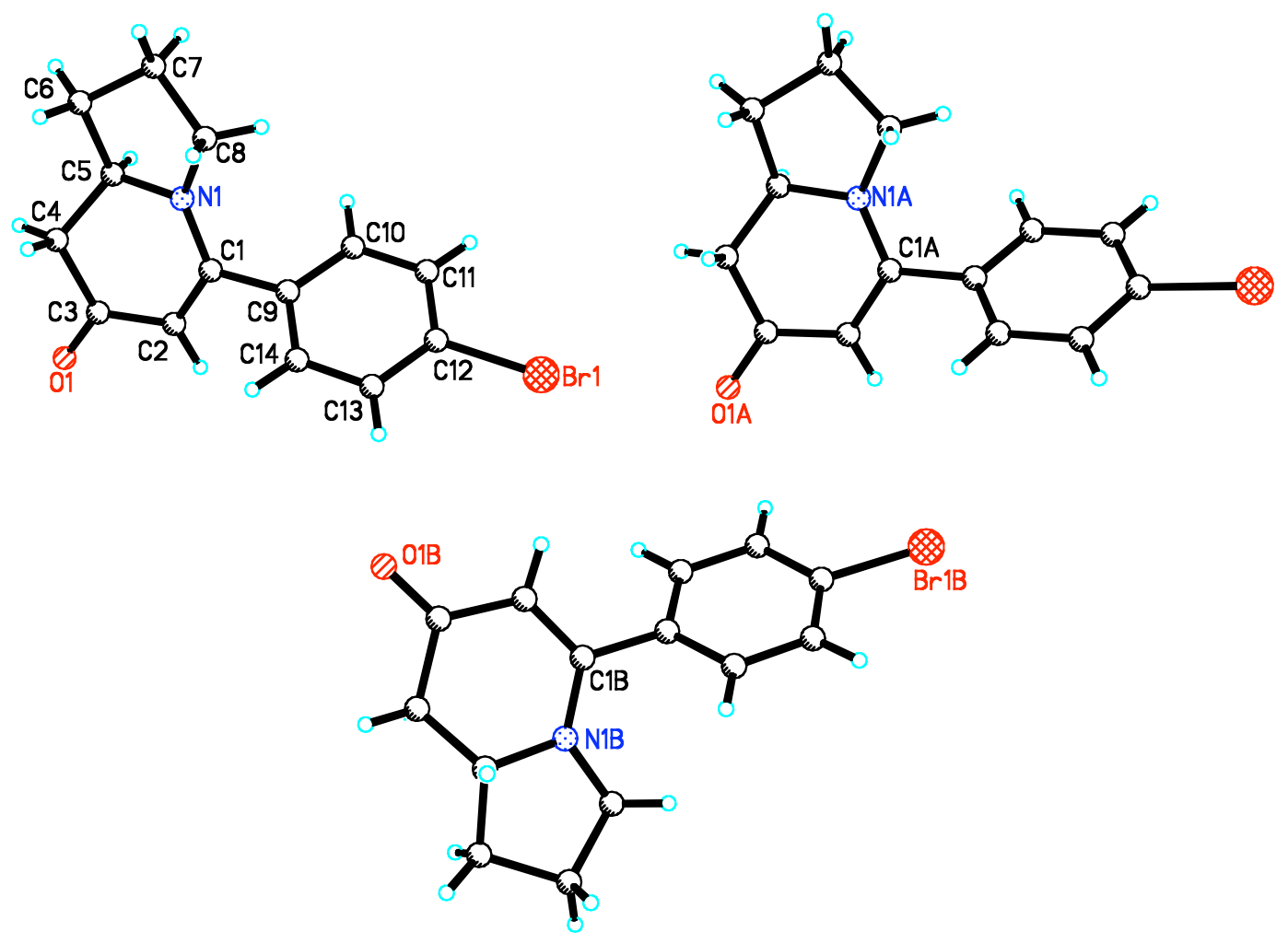


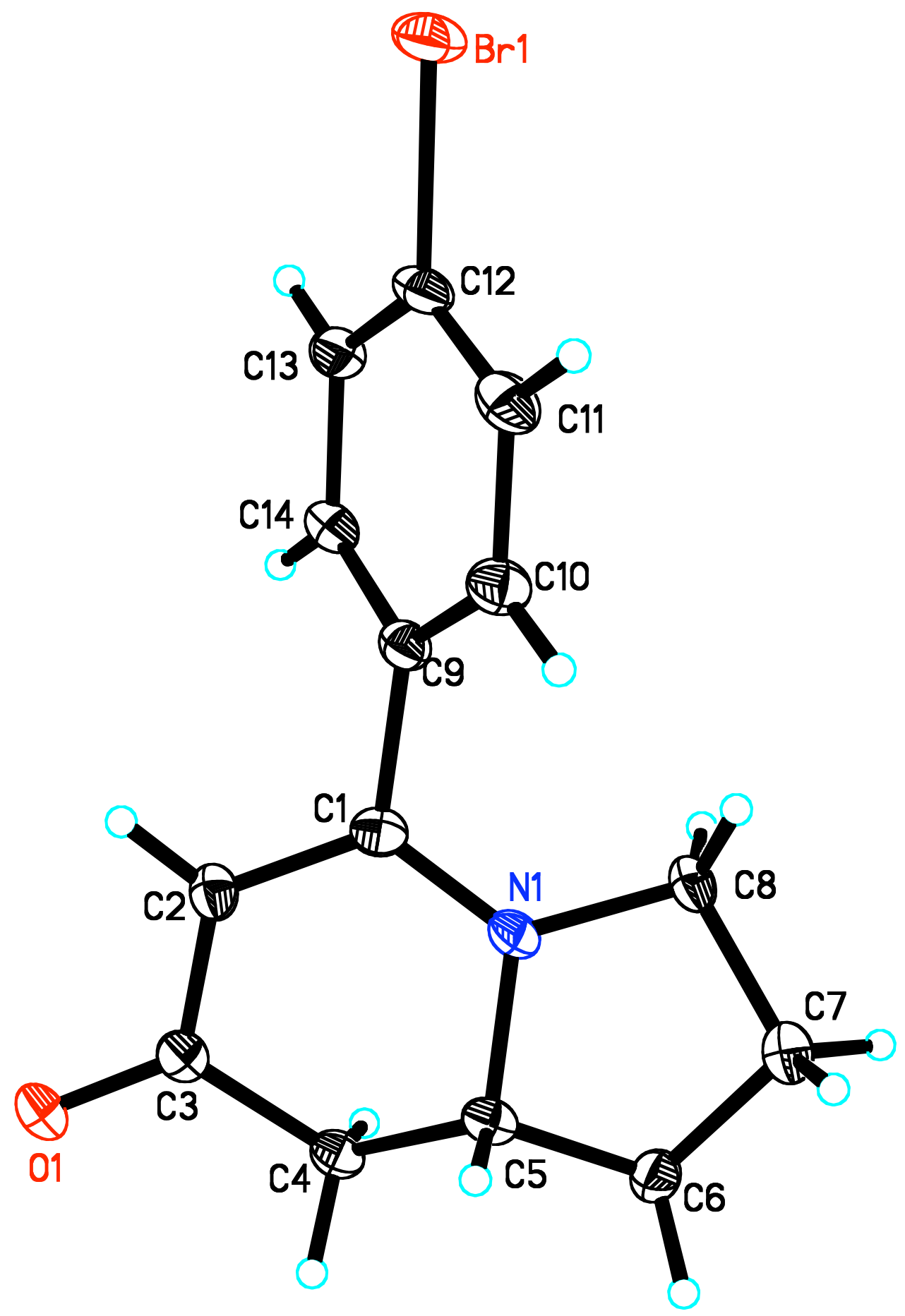




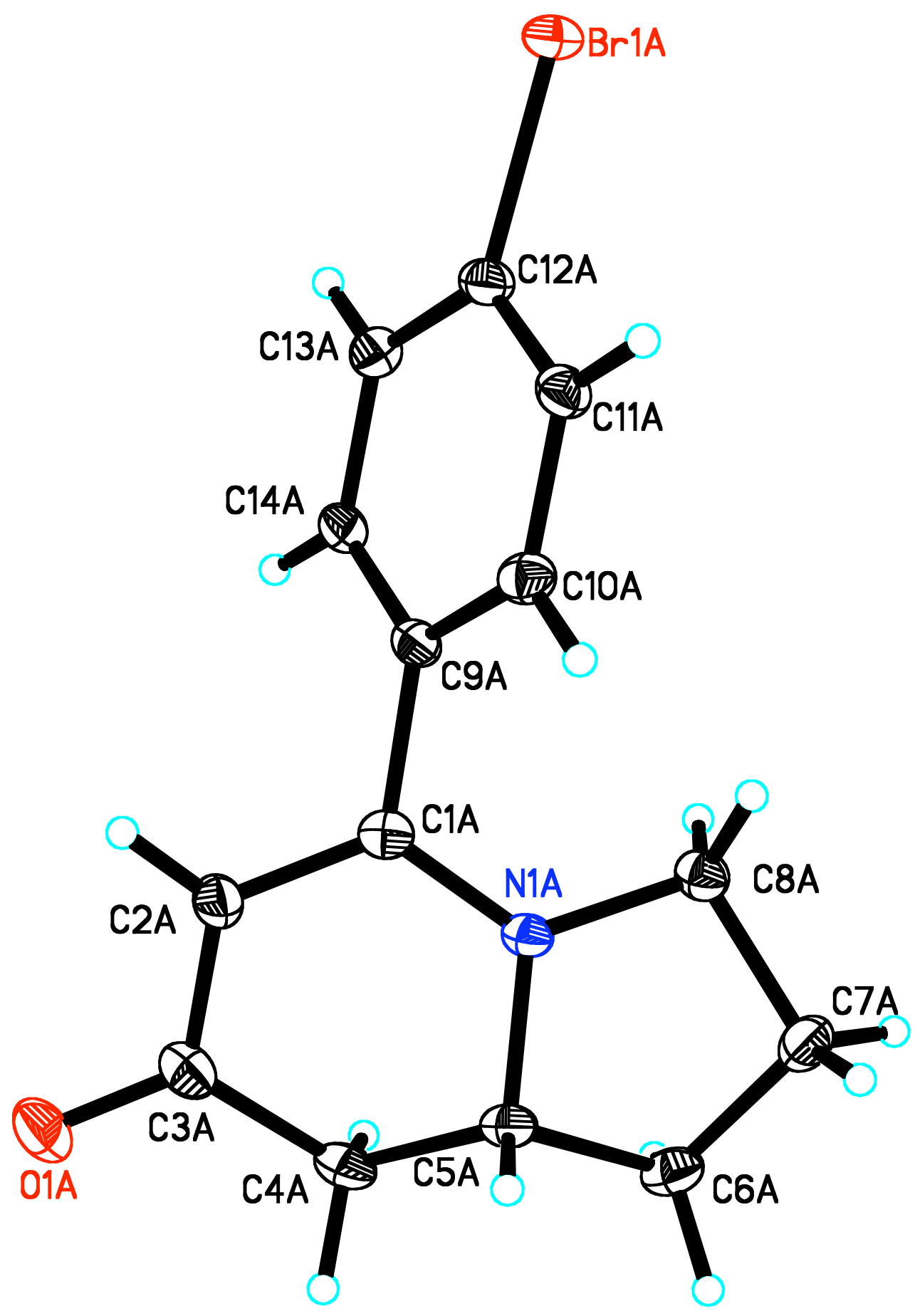




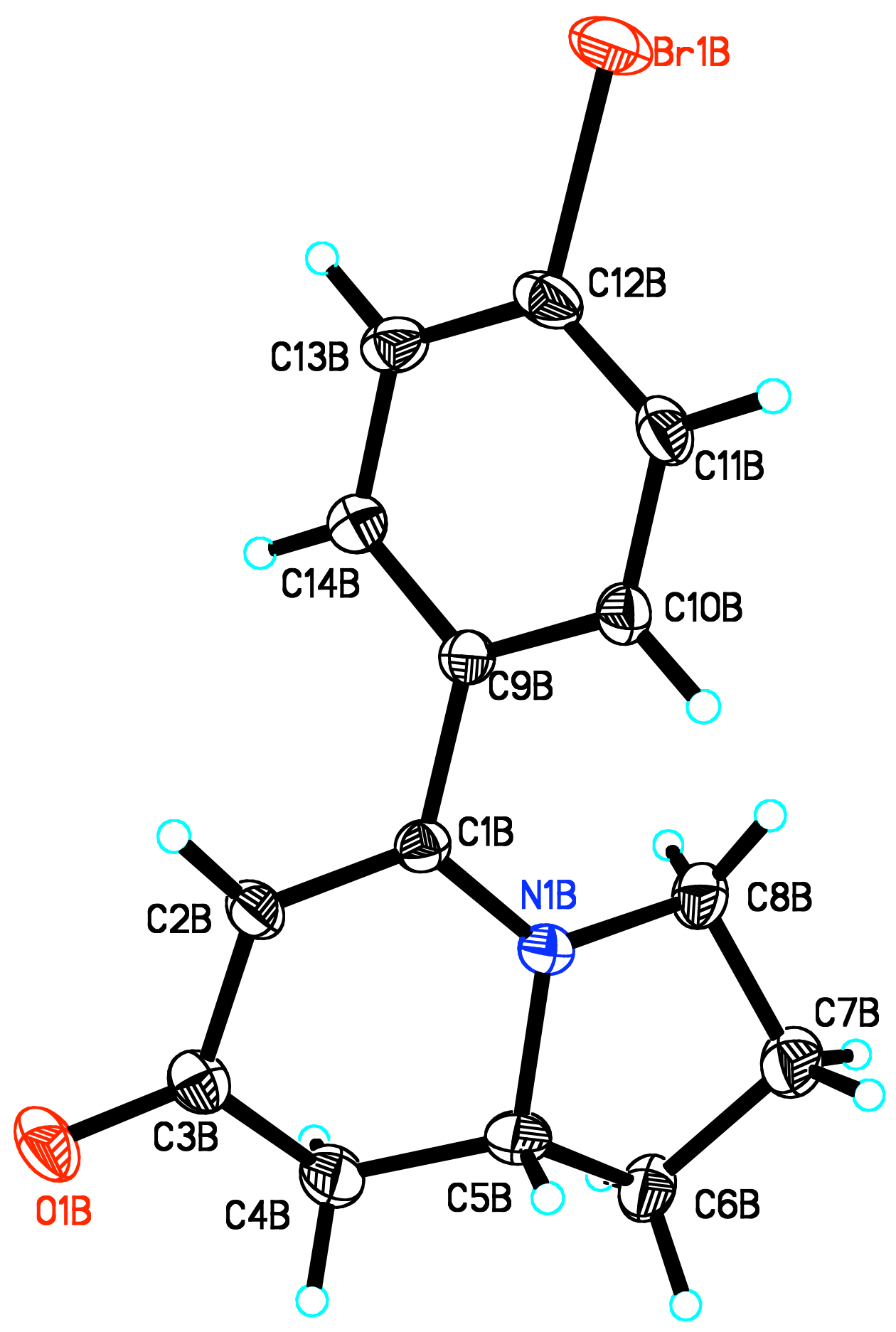

